Article

\title{
Inter-Variability Study of COVLIAS 1.0: Hybrid Deep Learning Models for COVID-19 Lung Segmentation in Computed Tomography
}

\author{
Jasjit S. Suri 1,2,*, Sushant Agarwal 2,3 (D), Pranav Elavarthi ${ }^{2,4}$, Rajesh Pathak ${ }^{5}$, Vedmanvitha Ketireddy ${ }^{6}$, \\ Marta Columbu ${ }^{7}$, Luca Saba ${ }^{7}$, Suneet K. Gupta ${ }^{8}$, Gavino Faa ${ }^{9}$ D, Inder M. Singh ${ }^{1}$, Monika Turk ${ }^{10}$, \\ Paramjit S. Chadha ${ }^{1}$, Amer M. Johri ${ }^{11}$, Narendra N. Khanna ${ }^{12}$, Klaudija Viskovic ${ }^{13}$, Sophie Mavrogeni ${ }^{14}$, \\ John R. Laird ${ }^{15}$, Gyan Pareek ${ }^{16}$, Martin Miner ${ }^{17}$, David W. Sobel ${ }^{16}$, Antonella Balestrieri ${ }^{7}$, Petros P. Sfikakis ${ }^{18}$, \\ George Tsoulfas ${ }^{19}$, Athanasios Protogerou ${ }^{20}\left(\mathbb{D}\right.$, Durga Prasanna Misra ${ }^{21}$, Vikas Agarwal ${ }^{21}$ (D), \\ George D. Kitas 22,23, Jagjit S. Teji ${ }^{24}$, Mustafa Al-Maini ${ }^{25}$, Surinder K. Dhanjil ${ }^{26}$, Andrew Nicolaides 27 , \\ Aditya Sharma ${ }^{28}$, Vijay Rathore ${ }^{26}$, Mostafa Fatemi ${ }^{29} \mathbb{D}$, Azra Alizad ${ }^{30}{ }^{\mathbb{D}}$, Pudukode R. Krishnan ${ }^{31}$, \\ Ferenc Nagy ${ }^{32}$, , Zoltan Ruzsa ${ }^{33}$, Archna Gupta ${ }^{34}$, Subbaram Naidu ${ }^{35}$ (i) and Mannudeep K. Kalra ${ }^{36}$
}

check for

updates

Citation: Suri, J.S.; Agarwal, S.; Elavarthi, P.; Pathak, R.; Ketireddy, V.; Columbu, M.; Saba, L.; Gupta, S.K.;

Faa, G.; Singh, I.M.; et al. Inter-Variability Study of COVLIAS 1.0: Hybrid Deep Learning Models for COVID-19 Lung Segmentation in Computed Tomography. Diagnostics 2021, 11, 2025. https://doi.org/ 10.3390/diagnostics11112025

Academic Editors: Sameer Antani and Sivaramakrishnan Rajaraman

Received: 30 September 2021 Accepted: 27 October 2021

Published: 1 November 2021

Publisher's Note: MDPI stays neutral with regard to jurisdictional claims in published maps and institutional affiliations.

Copyright: (c) 2021 by the authors. Licensee MDPI, Basel, Switzerland. This article is an open access article distributed under the terms and conditions of the Creative Commons Attribution (CC BY) license (https:/ / creativecommons.org/licenses/by/ $4.0 /)$.
1 Stroke Diagnostic and Monitoring Division, AtheroPoint ${ }^{\mathrm{TM}}$, Roseville, CA 95661, USA; drindersingh1@gmail.com (I.M.S.); pomchadha@gmail.com (P.S.C.)

2 Advanced Knowledge Engineering Centre, GBTI, Roseville, CA 95661, USA; sushant.ag09@gmail.com (S.A.); pmelavarthi@gmail.com (P.E.)

3 Department of Computer Science Engineering, PSIT, Kanpur 209305, India

4 Thomas Jefferson High School for Science and Technology, Alexandria, VA 22312, USA

5 Department of Computer Science Engineering, Rawatpura Sarkar University, Raipur 492001, India; drrkpathak20@gmail.com

6 Mira Loma High School, Sacramento, CA 95821, USA; manvi.ketireddy@gmail.com

7 Department of Radiology, Azienda Ospedaliero Universitaria (A.O.U.), 10015 Cagliari, Italy; martagiuliacol@gmail.com (M.C.); lucasabamd@gmail.com (L.S.); antonellabalestrieri@hotmail.com (A.B.)

8 Department of Computer Science, Bennett University, Noida 201310, India; suneet.gupta@bennett.edu.in

9 Department of Pathology, Azienda Ospedaliero Universitaria (A.O.U.), 10015 Cagliari, Italy; gavinofaa@gmail.com

10 The Hanse-Wissenschaftskolleg Institute for Advanced Study, 27753 Delmenhorst, Germany; monika.turk84@gmail.com

11 Department of Medicine, Division of Cardiology, Queen's University, Kingston, ON K7L 3N6, Canada; johria@queensu.ca

12 Department of Cardiology, Indraprastha APOLLO Hospitals, New Delhi 110076, India; drnnkhanna@gmail.com

13 University Hospital for Infectious Diseases, 10000 Zagreb, Croatia; klaudija.viskovic@bfm.hr

14 Cardiology Clinic, Onassis Cardiac Surgery Center, 10558 Athens, Greece; soma13@otenet.gr

15 Heart and Vascular Institute, Adventist Health St. Helena, St. Helena, CA 94574, USA; Lairdjr@ah.org

16 Minimally Invasive Urology Institute, Brown University, Providence, RI 02912, USA; gyan_pareek@brown.edu (G.P.); dwsobel@gmail.com (D.W.S.)

17 Men's Health Center, Miriam Hospital, Providence, RI 02906, USA; martin_miner@brown.edu

18 Rheumatology Unit, National \& Kapodistrian University of Athens, 10679 Athens, Greece; psfikakis@med.uoa.gr

19 Aristoteleion University of Thessaloniki, 54636 Thessaloniki, Greece; tsoulfasg@gmail.com

20 National \& Kapodistrian University of Athens, 10679 Athens, Greece; aprotog@med.uoa.gr

21 Department of Immunology, Sanjay Gandhi Postgraduate Institute of Medical Sciences, Lucknow 226014, India; durgapmisra@gmail.com (D.P.M.); vikasagr@yahoo.com (V.A.)

22 Academic Affairs, Dudley Group NHS Foundation Trust, Dudley DY1 2HQ, UK; george.kitas@nhs.net

23 Arthritis Research UK Epidemiology Unit, Manchester University, Manchester M13 9PT, UK

24 Ann and Robert H. Lurie Children's Hospital of Chicago, Chicago, IL 60611, USA; jteji@mercy-chicago.org

25 Allergy, Clinical Immunology and Rheumatology Institute, Toronto, ON L4Z 4C4, Canada; almaini@hotmail.com

26 AtheroPoint LLC, Roseville, CA 95611, USA; surinderdhanjil@gmail.com (S.K.D.);

Vijay.s.rathore@kp.org (V.R.)

27 Vascular Screening and Diagnostic Centre, University of Nicosia Medical School, Nicosia 2368, Cyprus; anicolaides1@gmail.com

28 Division of Cardiovascular Medicine, University of Virginia, Charlottesville, VA 22904, USA; AS8AH@hscmail.mcc.virginia.edu 
29 Department of Physiology \& Biomedical Engineering, Mayo Clinic College of Medicine and Science, Rochester, MN 55905, USA; fatemi.mostafa@mayo.edu

30 Department of Radiology, Mayo Clinic College of Medicine and Science, Rochester, MN 55905, USA; Alizad.Azra@mayo.edu

31 Neurology Department, Fortis Hospital, Bangalore 560076, India; prkrish12@rediffmail.com

32 Internal Medicine Department, University of Szeged, 6725 Szeged, Hungary; drnagytfer@hotmail.com

33 Zoltan Invasive Cardiology Division, University of Szeged, 6725 Szeged, Hungary; zruzsa@icloud.com

34 Radiology Department, Sanjay Gandhi Postgraduate Institute of Medical Sciences, Lucknow 226014, India; garchna@gmail.com

35 Electrical Engineering Department, University of Minnesota, Duluth, MN 55812, USA; dsnaidu@d.umn.edu

36 Department of Radiology, Massachusetts General Hospital, 55 Fruit Street, Boston, MA 02114, USA; mkalra@mgh.harvard.edu

* Correspondence: jasjit.suri@atheropoint.com; Tel.: +1-(916)-749-5628

Abstract: Background: For COVID-19 lung severity, segmentation of lungs on computed tomography (CT) is the first crucial step. Current deep learning (DL)-based Artificial Intelligence (AI) models have a bias in the training stage of segmentation because only one set of ground truth (GT) annotations are evaluated. We propose a robust and stable inter-variability analysis of CT lung segmentation in COVID-19 to avoid the effect of bias. Methodology: The proposed inter-variability study consists of two GT tracers for lung segmentation on chest CT. Three AI models, PSP Net, VGG-SegNet, and ResNet-SegNet, were trained using GT annotations. We hypothesized that if AI models are trained on the GT tracings from multiple experience levels, and if the AI performance on the test data between these AI models is within the $5 \%$ range, one can consider such an AI model robust and unbiased. The K5 protocol (training to testing: 80\%:20\%) was adapted. Ten kinds of metrics were used for performance evaluation. Results: The database consisted of $5000 \mathrm{CT}$ chest images from 72 COVID-19-infected patients. By computing the coefficient of correlations (CC) between the output of the two AI models trained corresponding to the two GT tracers, computing their differences in their CC, and repeating the process for all three AI-models, we show the differences as $0 \%, 0.51 \%$, and $2.04 \%($ all $<5 \%)$, thereby validating the hypothesis. The performance was comparable; however, it had the following order: ResNet-SegNet > PSP Net > VGG-SegNet. Conclusions: The AI models were clinically robust and stable during the inter-variability analysis on the CT lung segmentation on COVID-19 patients.

Keywords: COVID-19; computed tomography; lungs; variability; segmentation; hybrid deep learning

\section{Introduction}

The WHO's International Health Regulations and Emergency Committee (IHREC) proclaimed COVID-19 a "public health emergency of international significance" or "pandemic" on 30 January 2020. More than 231 million people have been infected worldwide, and nearly 4.7 million people have died due to COVID-19 [1]. Although this "severe acute respiratory syndrome coronavirus 2" (SARS-CoV-2) virus specifically targets the pulmonary and vascular system, it has the potential to travel through the body and lead to complications such as pulmonary embolism [2], myocardial infarction, stroke, or mesenteric ischemia [3-5]. Comorbidities such as diabetes mellitus, hypertension, and obesity substantially increase the severity and mortality of COVID-19 [6,7]. A real-time reverse transcription-polymerase chain reaction (RT-PCR) is the recommended method for diagnosis [8]. Chest radiographs and computed tomography (CT) [9-11] are used to determine disease severity in patients with moderate to severe disease or underlying comorbidities based on the extent of pulmonary opacities such as ground-glass (GGO), consolidation, and mixed opacities in CT scans [7,12-14].

Most radiologists provide a semantic description of the extent and type of opacities to describe the severity of COVID-19 pneumonia. The semiquantitative evaluation of pulmonary opacities is time-consuming, subjective, and tedious [15-18]. Thus, there is a need for a fast and error-free early COVID-19 disease diagnosis and real-time prognosis 
solutions. Machine learning (ML) offers a solution to this problem by providing a rich set of algorithms [19]. Previously, ML has been used for detection of cancers in breast [20], liver [21,22], thyroid [23-25], skin [26,27], prostate [28,29], ovary [30], and lung [31]. There are two main components in disease detection, i.e., segmentation [32-35] and classification [36,37], where segmentation plays a crucial step. An extension of ML called deep learning (DL) employs dense layers to automatically extract and classify all relevant imaging features [38-43]. Hybrid DL (HDL), a method that combines two DL systems, helps address some of the challenges in solo DL models [44,45]. This includes overfitting and optimization of hyperparameters, thereby removing the bias [45].

During the AI model training, the most crucial stage is the ground truth (GT) annotation of organs that need to be segmented. It is a time-consuming operation with monetary constraints since skilled personnel such as radiologists are expensive to recruit and difficult to find. These annotations, if conducted by one tracer, make the AI system biased. A plurality of tracers being used to produce the GT annotated dataset makes the system more resilient and lowers the AI bias [46-49]. This is because the AI model can grasp and adjust to the sensitivity of the difference in the tracings of the tracers. Thus, to avoid AI bias, one needs to have an automated AI-based system with multiple tracers. To establish the validity of such automated AI systems, one must undergo inter-variability analysis with two or more observers.

To validate the AI systems, we hypothesize that two conditions must be met: (a) the two observers should perform within $5 \%$ range of each other and (b) the performance of the AI system using the ground truth tracings from these two observers should also be within the 5\% threshold [48]. The AI performance is computed between the GT-area and the AI model-estimated area. The focus of the proposed research is to design a reliable AI system based on the inter-observer paradigm. Figure 1 depicts a COVID-19 CT lung segmentation system in which the CT machine is used to acquire CT volumes. This volume is then annotated by multiple observers (Figure 1, $n$ denotes the number of observers), and multiple AI models are generated, which is then used for lung segmentation. The segmentation output is the binary mask of the lung, its boundary, and the corresponding boundary overlays. This output can be used for evaluating the performance, analysis, and quantification of the results.

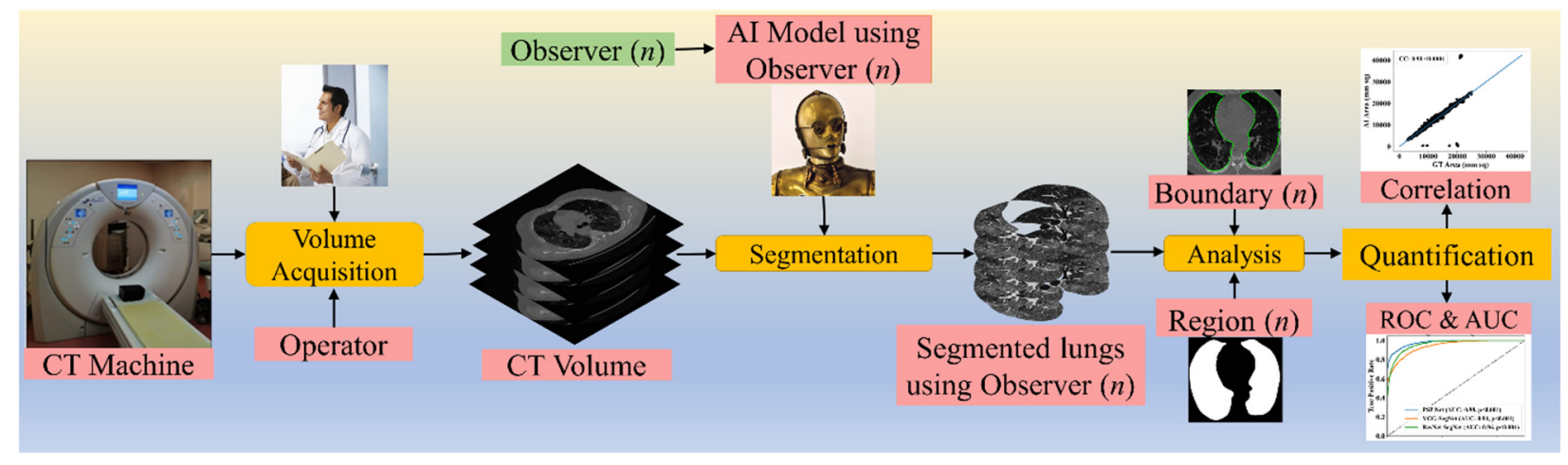

Figure 1. COVLIAS 1.0: Inter-variability analysis of CT-based lung segmentation and quantification system for COVID-19 patients. ROC: Receiver operating characteristic; AUC: Area-under-the-curve.

The layout of this inter-variability study is as follows: Section 2 presents the methodology with the demographics, COVLIAS 1.0 pipeline, AI architectures, and loss functions. The experimental protocol is shown in Section 3, while results and performance evaluation are presented in Section 4. The discussions and conclusions are presented in Sections 5 and 6, respectively. 


\section{Methodology}

\subsection{Patient Demographics, Image Acquisition, and Data Preparation}

\subsubsection{Demographics}

The dataset consists of 72 adult Italian patients with 46 being male and the remaining being female. The mean height and weight were $173 \mathrm{~cm}$ and $79 \mathrm{~kg}$, respectively. A total of 60 patients tested positive for RT-PCR, while 12 patients were confirmed using broncho-alveolar lavage [50]. Overall, the cohort had an average of $4.1 \mathrm{GGO}$, which was considered low.

\subsubsection{Image Acquisition}

All chest $\mathrm{CT}$ scans were performed in a supine posture during a single full inspiratory breath-hold using a 128-slice multidetector-row Philips Healthcare's "Philips Ingenuity Core" CT scanner. There were no intravenous or oral contrast media administrations. The CT exams were performed using a $120 \mathrm{kV}, 226 \mathrm{mAs} /$ slice (utilizing an automatic tube current modulation-Z-DOM by Philips), a 1.08 spiral pitch factor, 0.5 -s gantry rotation time, and $64 \times 0.625$ detector setup. Soft tissue kernel with $512 \times 512$ matrix (mediastinal window) and lung kernel with $768 \times 768$ matrix (lung window) was used to reconstruct $1 \mathrm{~mm}$-thick images. The Picture Archiving and Communication System (PACS) workstation that was utilized to review the CT images was outfitted with two Eizo $35 \times 43 \mathrm{~cm}$ displays with a $2048 \times 1536$ matrix. Figure 2 shows the raw sample CT scans of COVID-19 patients with varying lung sizes and variable intensity patterns, posing a challenge.

\subsubsection{Data Preparation}

The proposed study makes use of the CT data of 72 COVID-positive individuals. Each patient had 200 slices, out of which the radiologist [LS] chose 65-70 slices from the visible lung region, resulting in 5000 images in total. The AI-based segmentation models were trained and tested using these 5000 images. To prepare the data for segmentation, a binary mask was created manually in a selected slice with the help of ImgTracer ${ }^{\mathrm{TM}}$ under the supervision of a qualified radiologist [LS] (Global Biomedical Technologies, Inc., Roseville, CA, USA) $[47,48,51]$. Figure 3 shows the white binary mask of the lung region computed using ImgTracer ${ }^{\mathrm{TM}}$ during manual tracings by Observer 1 and 2 (both were postgraduate researchers trained by our radiological team).

\subsection{Architecture}

COVLIAS 1.0 system incorporates three models: one solo DL (SDL) and two hybrid DL (HDL). The proposed study incorporates three AI models: (a) PSP Net, (b) VGG-SegNet, and (c) ResNet-SegNet.

\subsubsection{Three AI Models: PSP Net, VGG-SegNet, and ResNet-SegNet}

The Pyramid Scene Parsing Network (PSP Net) [52] is a semantic segmentation network with the ability to consider the global context of the image. The architecture of PSP Net (Figure 4) has four parts: (i) input, (ii) feature map, (iii) pyramid pooling module, and (iv) output. The input to the network is the image to be segmented, which undergoes extraction of the feature map using a set of dilated convolution and pooling blocks. The dilated convolution layer is added at the last two blocks of the network to keep more prominent features at the end. The next stage is the pyramid pooling module; it is the heart of the network, as it helps capture the global context of the image/feature map generated in the previous step. This section consists of four parts, each with a different scaling ability. The scaling of this module includes $1,2,3$, and 6 , where $1 \times 1$ scaling helps capture the spatial features and thereby increases the resolution of the features captured. The $6 \times 6$ scaling captures the higher-resolution features. At the end of this module, all the output from these four parts is pooled using global average pooling. For the last part, the global average pooling output is fed to a set of convolutional layers. Finally, the set of prediction classes are generated as the output binary mask. 


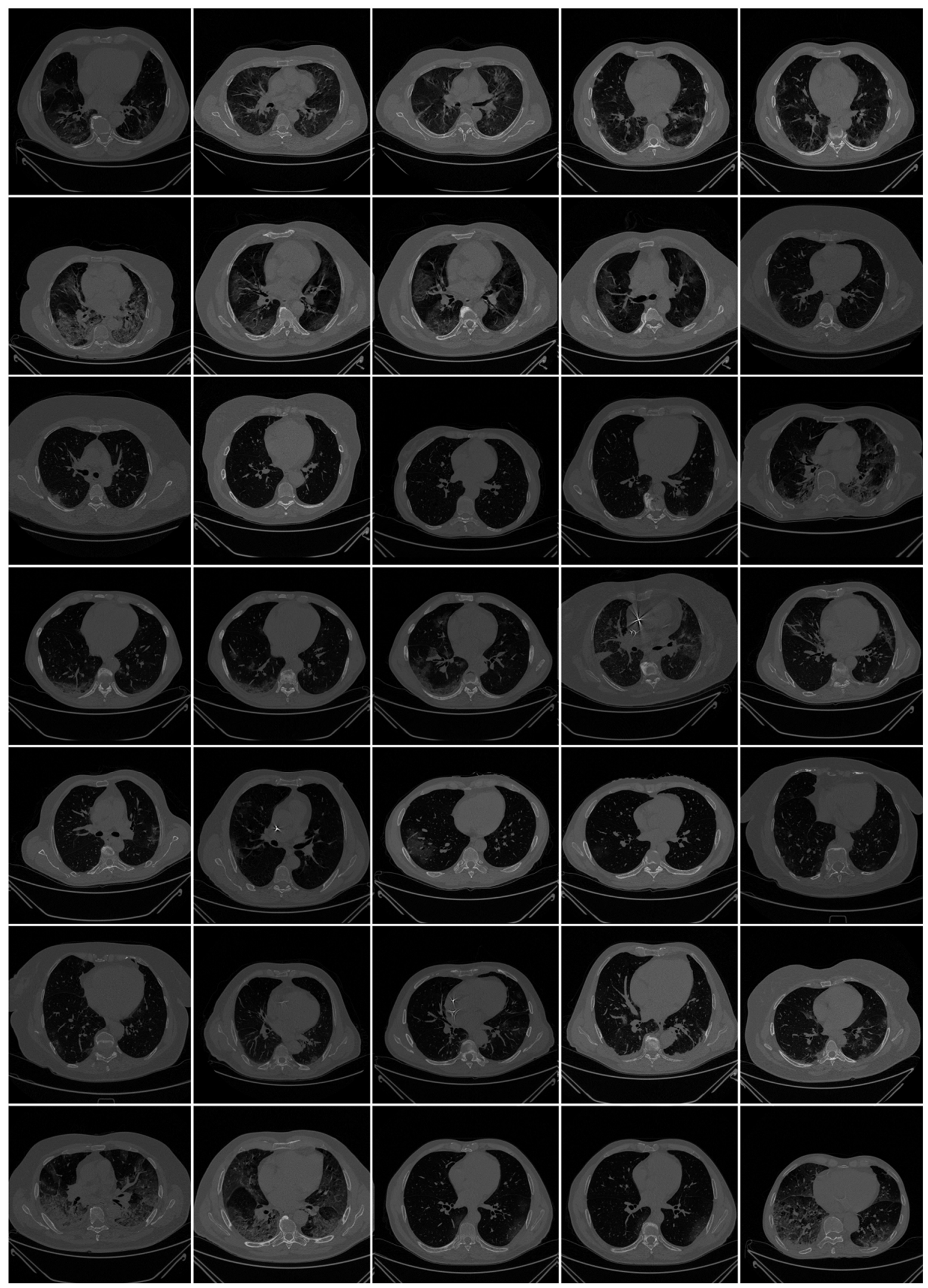

Figure 2. Raw lung COVID-19 CT scans taken from different patients in the database. 


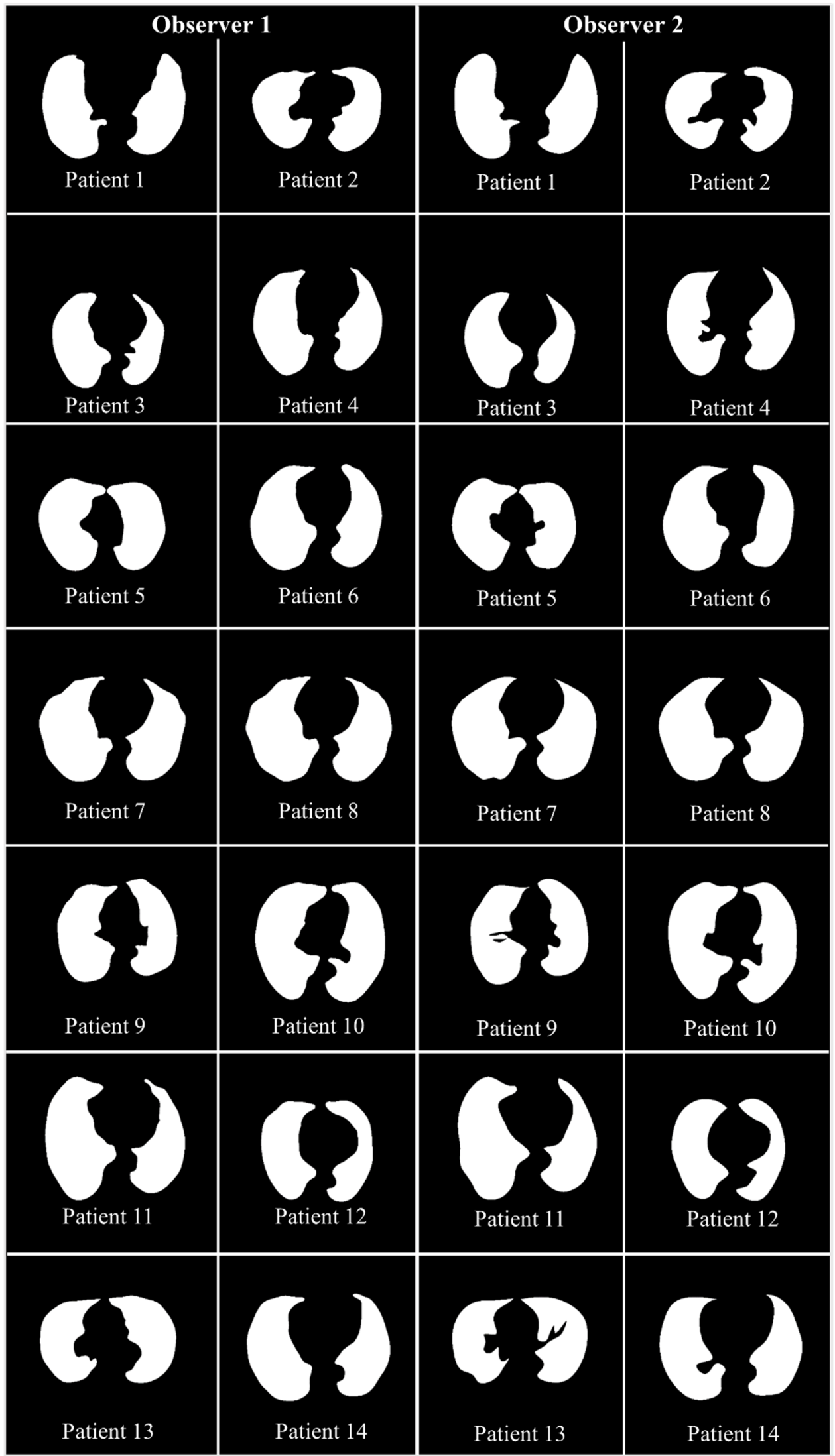

Figure 3. GT white binary mask for AI model training for Observer 1 vs. Observer 2. 


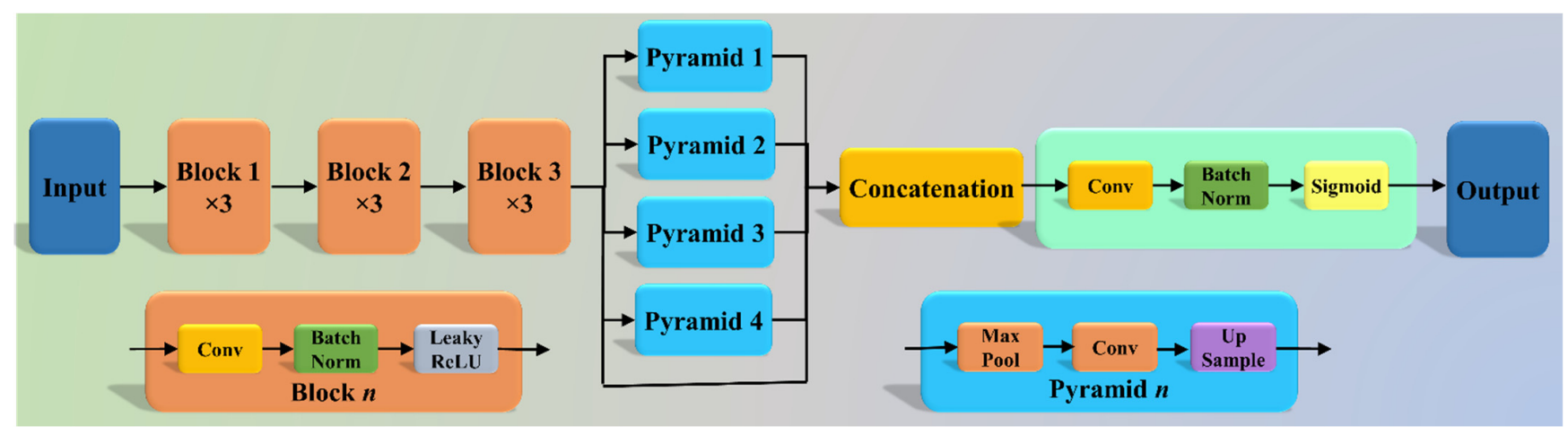

Figure 4. PSP Net architecture.

The VGGNet architecture (Figure 5) was designed to reduce the training time by replacing the kernel filter in the initial layer with an 11 and 5 sized filter, thereby reducing the \# of parameters in the two-dimension convolution (Conv) layers [53]. The VGG-SegNet architecture used in this study is composed of three parts (i) encoder, (ii) decoder part, and (iii) a pixel-wise SoftMax classifier at the end. It consists of 16 Conv layers compared to the SegNet architecture, where only 13 Conv layers are used [54] in the encoder part. This increase in \#layers helps the model extract more features from the image. The final output of the model is a binary mask with the lung region annotated as 1 (white) and the rest of the image as 0 (black).

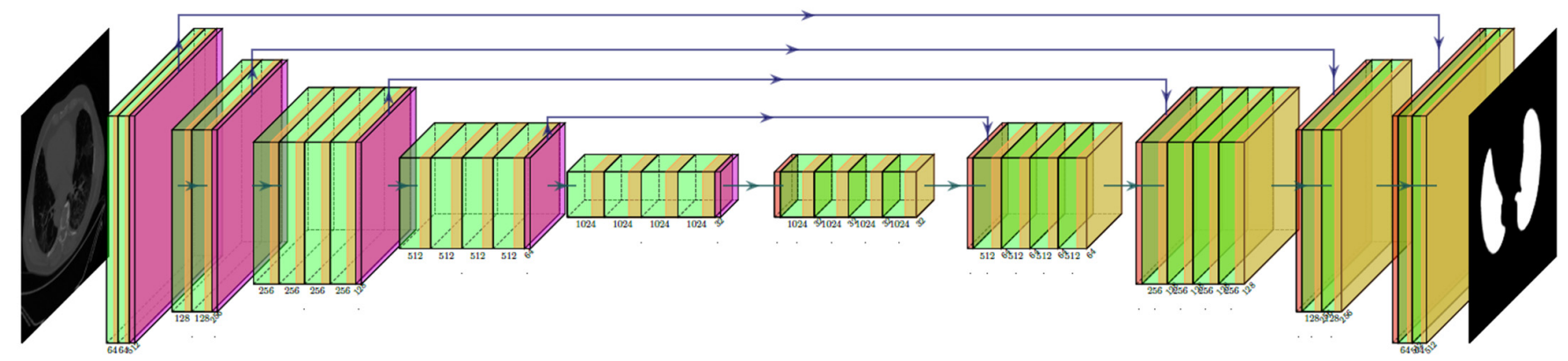

Figure 5. VGG-SegNet architecture.

Although VGGNet was very efficient and fast, it suffered from the problem of vanishing gradients. It results in significantly less or no weight training during backpropagation; at each epoch, it keeps getting multiplied with the gradient, and the update to the initial layers is very small. To overcome this problem, Residual Network or ResNet [55] came into existence (Figure 6). In this architecture, a new connection was introduced known as skip connection which allowed the gradients to bypass a certain number of layers, solving the vanishing gradient problem. Moreover, with the help of one more additions to the network, i.e., an identity function, the local gradient value was kept to one during the backpropagation step.

\subsubsection{Loss Functions for AI Models}

The proposed system uses cross-entropy (CE)-loss during the training of the AI models. Equation (1) below represents the CE-loss, symbolized as $l_{C E}$, for the three AI models:

$$
l_{C E}=-\left[\left(x_{\mathrm{i}} \times \log \mathrm{p}_{\mathrm{i}}\right)+\left(1-x_{\mathrm{i}}\right) \times \log \left(1-\mathrm{p}_{\mathrm{i}}\right)\right]
$$

where $x_{\mathrm{i}}$ represents the input GT label $1,\left(1-x_{\mathrm{i}}\right)$ represents the GT label $0, \mathrm{p}_{\mathrm{i}}$ represents the probability of the classifier (SoftMax) used at the last layer of the AI model, and $\times$ represents the product of the two terms. Figures 4-6 presents the three AI architectures that have been trained using the CE-loss function. 


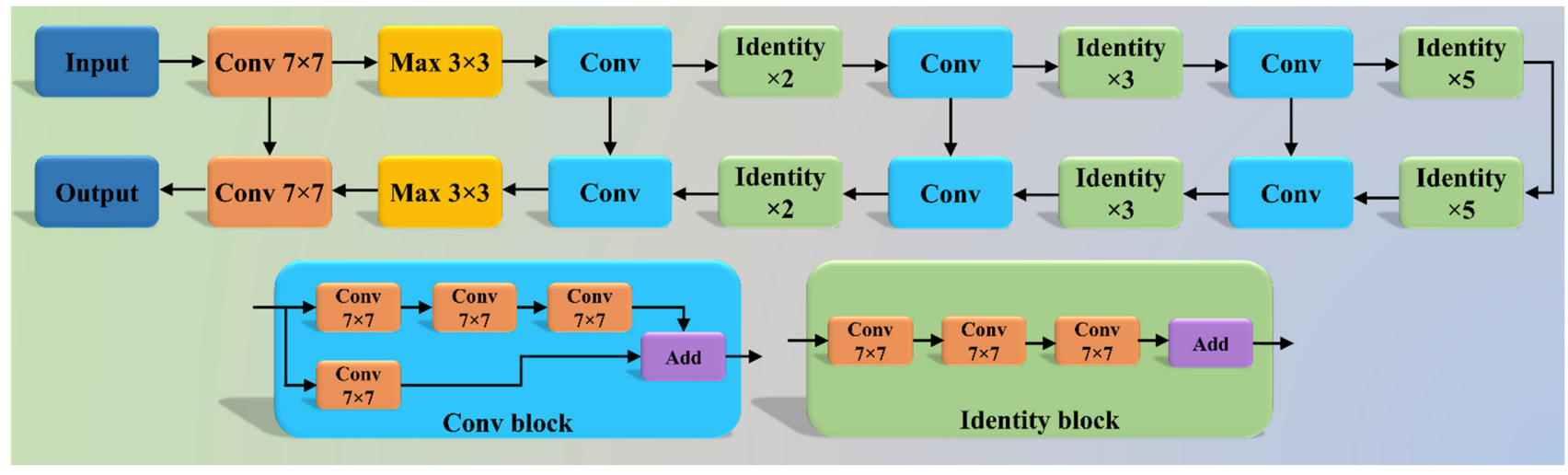

Figure 6. ResNet-SegNet architecture.

\section{Experimental Protocol}

\subsection{Accuracy Estimation of AI Models Using Cross-Validation}

A standardized cross-validation (CV) protocol was adapted for determining the accuracy of the AI models. Our group has published several CV-based protocols of different kinds using AI framework $[27,30,37,56,57]$. Since the data were moderate, the K5 protocol was used, which consisted of $80 \%$ training data (4000 CT images) and 20\% testing (1000 CT images). Five folds were designed in such a way that each fold got a chance to have a unique test set. An internal validation mechanism was part of the K5 protocol where $10 \%$ data was considered for validation.

\subsection{Lung Quantification}

There were two methods used for quantification of the segmented lungs using AI models. The spirit of these two methods originates from the shape analysis concept. In the first method, lung area (LA) is computed since the region is balloon-shaped, thus the area parameter is well suited for the measurement $[58,59]$. In the second method, we compute the long-axis of the lung (LLA) since the shape of the lung is more longitudinal than circular. A similar approach was taken for the long-axis view in heart computation [60]. The lung area (LA) was calculated by counting the number of white pixels in the binary mask segmented lungs, and the lung long axis (LLA) was calculated by the most distant distance segment joining anterior to posterior of the lungs. A resolution factor of 0.52 was used to convert (i) pixel to $\mathrm{mm}^{2}$ for the LA and (ii) pixel to $\mathrm{mm}$ for the LLA computation and quantification.

If the total number of the image is represented by $N$ in the database, $A_{a i}(m, n)$ represents lung area for in the image " $n$ " using the AI model " $m$ ", $\bar{A}_{a i}(m)$ represents the mean lung area corresponding to the AI model " $\mathrm{m}$," and mean area of the GT binary mask is represented by $\bar{A}_{g t}$, then mathematically $\bar{A}_{a i}(m)$ and $\bar{A}_{g t}$ can be computed as shown in Equation (2).

$$
\left.\begin{array}{c}
\bar{A}_{a i}(m)=\frac{\sum_{n=1}^{N} A_{a i}(m, n)}{N} \\
\bar{A}_{g t}=\frac{\sum_{n=1}^{N} A_{g t}(n)}{N}
\end{array}\right\}
$$

Similarly, $L A_{a i}(m, n)$ represents LLA for in the image " $n$ " using the AI model " $m$ ", $\overline{L A}_{a i}(m)$ represents the mean LLA corresponding to the AI model " $\mathrm{m}, " \overline{L A}_{g t}$ represents the corresponding mean LLA of the GT binary lung mask, then mathematically $\overline{L A}_{a i}(m)$ and $\overline{L A}_{g t}$ can be computed as shown in Equation (3).

$$
\left.\begin{array}{c}
\overline{L A}_{a i}(m)=\frac{\sum_{n=1}^{N} L A_{a i}(m, n)}{N} \\
\overline{L A}_{g t}=\frac{\sum_{n=1}^{N} L A_{g t}(n)}{N}
\end{array}\right\}
$$




\subsection{AI Model Accuracy Computation}

The accuracy of the AI system was measured by comparing the predicted output and the ground truth pixel values. These values were interpreted as binary ( 0 or 1$)$ numbers as the output lung mask was only black and white, respectively. Finally, these binary numbers were summed up and divided by the total number of pixels in the image. If TP, TN, FN, and FP represent true positive, true negative, false negative, and false positive, then the accuracy of the AI system can be computed as shown in Equation (4) [61].

$$
\operatorname{ACC}(\mathrm{ai})(\%)=\left(\frac{\mathrm{TP}+\mathrm{TN}}{\mathrm{TP}+\mathrm{FN}+\mathrm{TN}+\mathrm{FP}}\right) \times 100
$$

\section{Results and Performance Evaluation}

\subsection{Results}

Previously, COVLIAS 1.0 [54] was designed to run on a training: testing ratio of 2:3 dataset from 5000 images. However, this study proposes an inter-observer variability study with $\mathrm{K} 5$ in a $\mathrm{CV}$ framework. The training was performed on two sets of annotations, i.e., Observer 1 and Observer 2 . The output results are similar to the previously published study, i.e., a binary mask of the segmented lungs. Figures 7-9 show the AI-generated binary mask, segmented lung, and color segmented lung with grayscale background as an overlay for the three AI models.
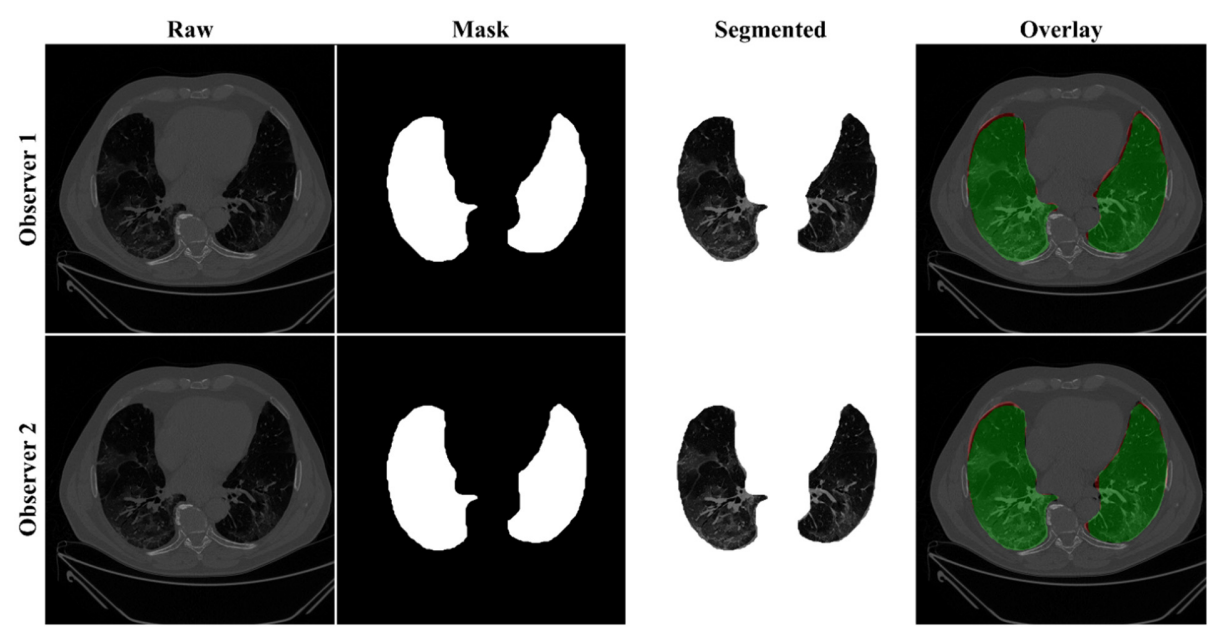

Figure 7. Results from PSP Net while using Observers 1 and 2. Columns are the raw, binary mask output, segmented lung region, and overlay of the estimated lung region vs. ground truth region.

\subsection{Performance Evaluation}

This section deals with the performance evaluation (PE) of the three AI models for Observer 1 vs. Observer 2. Section 4.2.1 presents the visual comparison of the results, which includes (i) boundary overlays against the ground truth boundary and (ii) lung long axis against the ground truth axis. Section 4.2.2 shows the PE for lung area error, which consists of (i) cumulative frequency (CF) plot, (ii) Bland-Altman plot, (iii) Jaccard Index (JI) and Dice Similarity (DS), and (iv) ROC and AUC curves for the three AI-based models' performance for Observer 1 vs. Observer 2. Similarly, lung long axis error (LLAE) presents PE using (i) cumulative plot, (ii) correlation coefficient (CC), and (iii) Bland-Altman plot. Finally, statistical analyses of the LA and LLA are presented using paired $t$-test, Wilcoxon, MannWhitney, and CC values for all 12 possible combinations for three AI models between Observer 1 and Observer 2. 

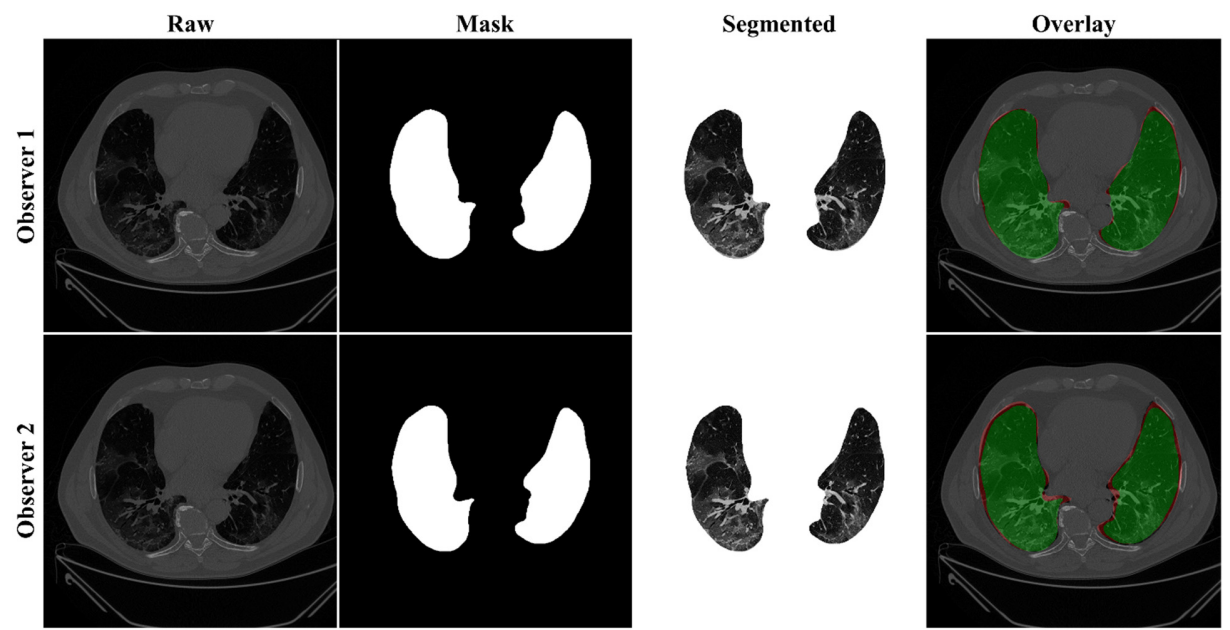

Figure 8. Results from VGG-SegNet while using Observers 1 and 2. Columns are the raw, binary mask output, segmented lung region, and overlay of the estimated lung region vs. ground truth region.
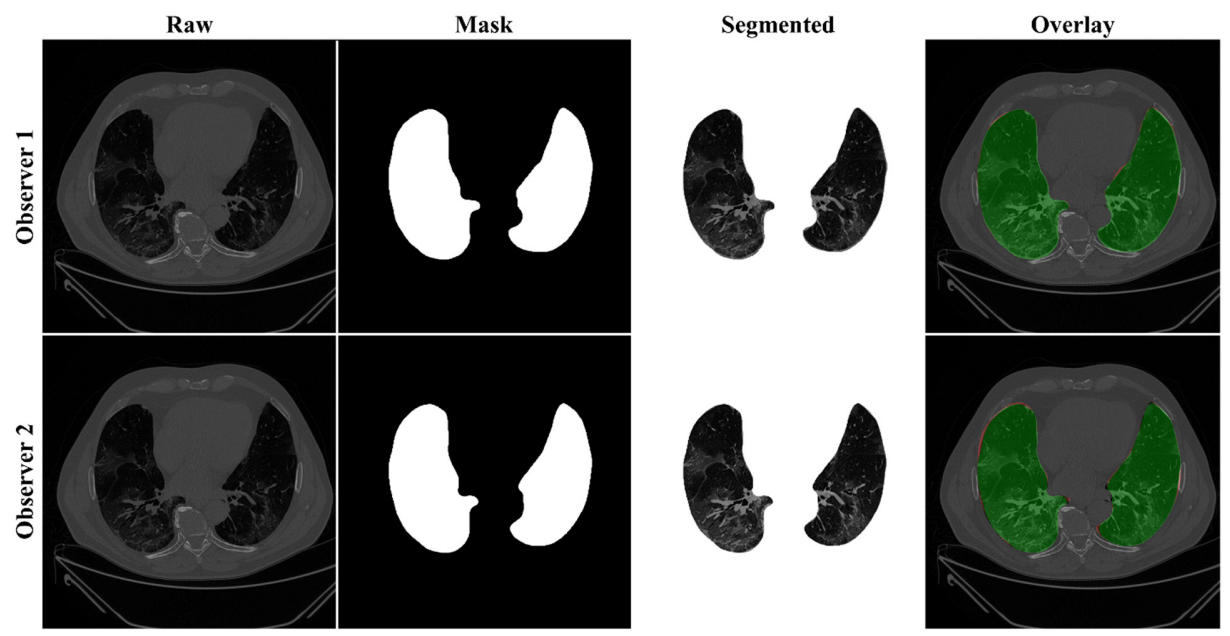

Figure 9. Results from ResNet-SegNet while using Observers 1 and 2. Columns are the raw, binary mask output, segmented lung region, and overlay of the estimated lung region vs. ground truth region.

\subsubsection{Lung Boundary and Long Axis Visualization}

The overlay for the three AI model boundaries (green) and GT-boundary (red) corresponding to Observer 1 (left) and Observer 2 (right) with a grayscale COVID-19 CT slice in the background is shown in Figure 10, while Figure 11 shows the AI-long axis (green) and GT-long axis (red) between Observer 1 and Observer 2 for three AI models. It shows the reach of anterior to posterior of the left and right lungs, with the GT boundary (white) corresponding to Observer 1 (left) and Observer 2 (right) of the lungs by the tracer using ImgTracer ${ }^{\mathrm{TM}}$. The three AI models follow the order: PSP Net, VGG-SegNet, and ResNet-SegNet.

\subsubsection{Performance Metrics for the Lung Area Error}

Cumulative Frequency Plot for Lung Area Error

The frequency of occurrence of the LAE is compared to a reference value in the cumulative frequency analysis and shown in Figure 12 (left lung) and Figure 13 (right lung) for three AI models between Observer 1 and Observer 2. A cutoff-score of $80 \%$ was chosen to show the difference between the three AI models. The LAE with the selected cutoff for the left lung was $1123.36 \mathrm{~mm}^{2}, 725.90 \mathrm{~mm}^{2}$, and $571.65 \mathrm{~mm}^{2}$ for the three AI models using Observer 1, and $834.08 \mathrm{~mm}^{2}, 1730.58 \mathrm{~mm}^{2}$, and $683.42 \mathrm{~mm}^{2}$, respectively, for the three AI 
models using Observer 2. A similar trend was followed by the right lung with $1158.93 \mathrm{~mm}^{2}$, $612.47 \mathrm{~mm}^{2}$, and $532.44 \mathrm{~mm}^{2}$ for the three AI models using Observer 1, and $809.77 \mathrm{~mm}^{2}$, $1610.15 \mathrm{~mm}^{2}$, and $572.56 \mathrm{~mm}^{2}$, respectively, for the three AI models using Observer 2. The three AI models follow the order: PSP Net, VGG-SegNet, and ResNet-SegNet.

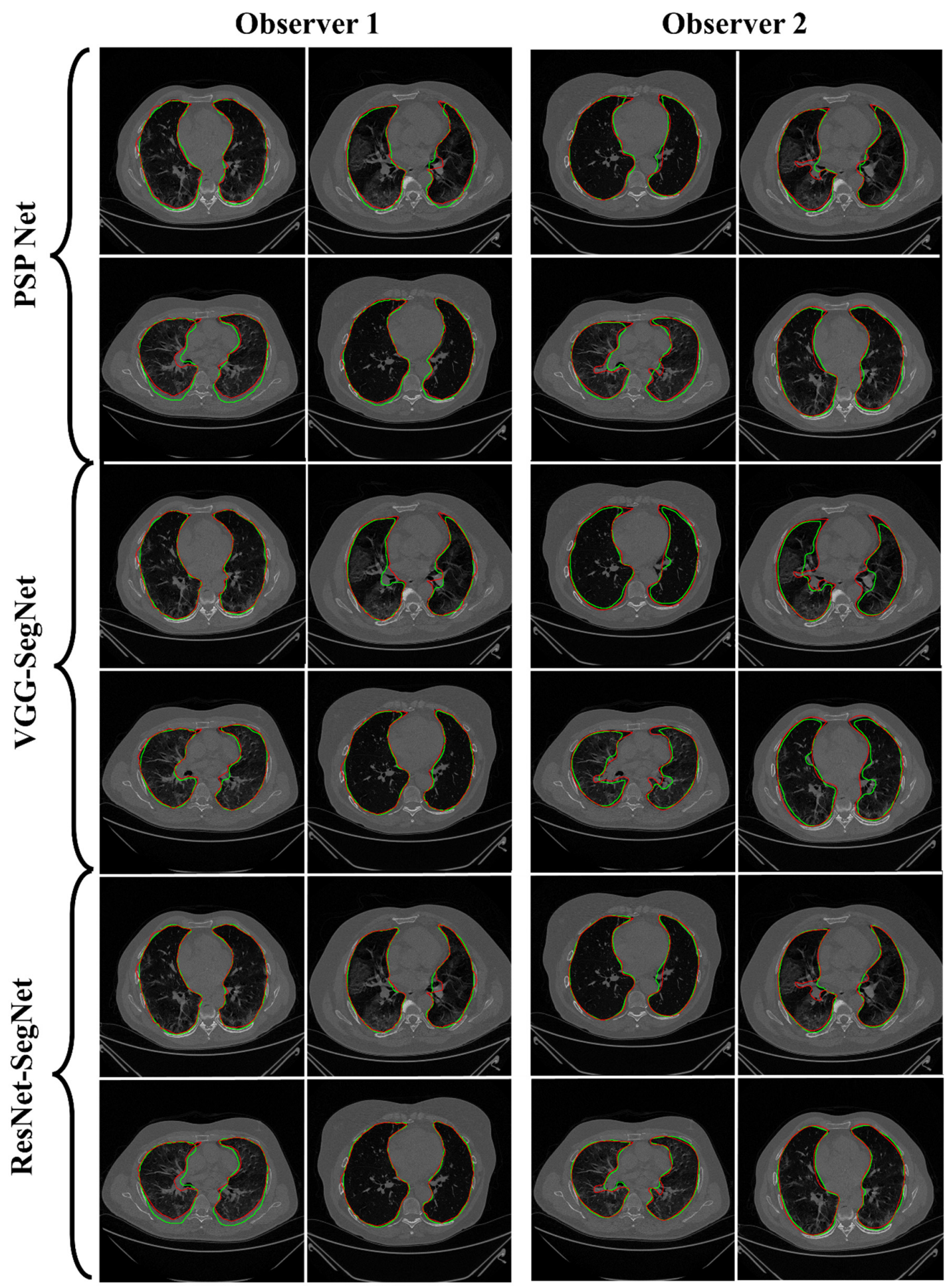

Figure 10. AI-model segmented boundary (green) vs. GT boundary (red) for Observer 1 and Observer 2. 

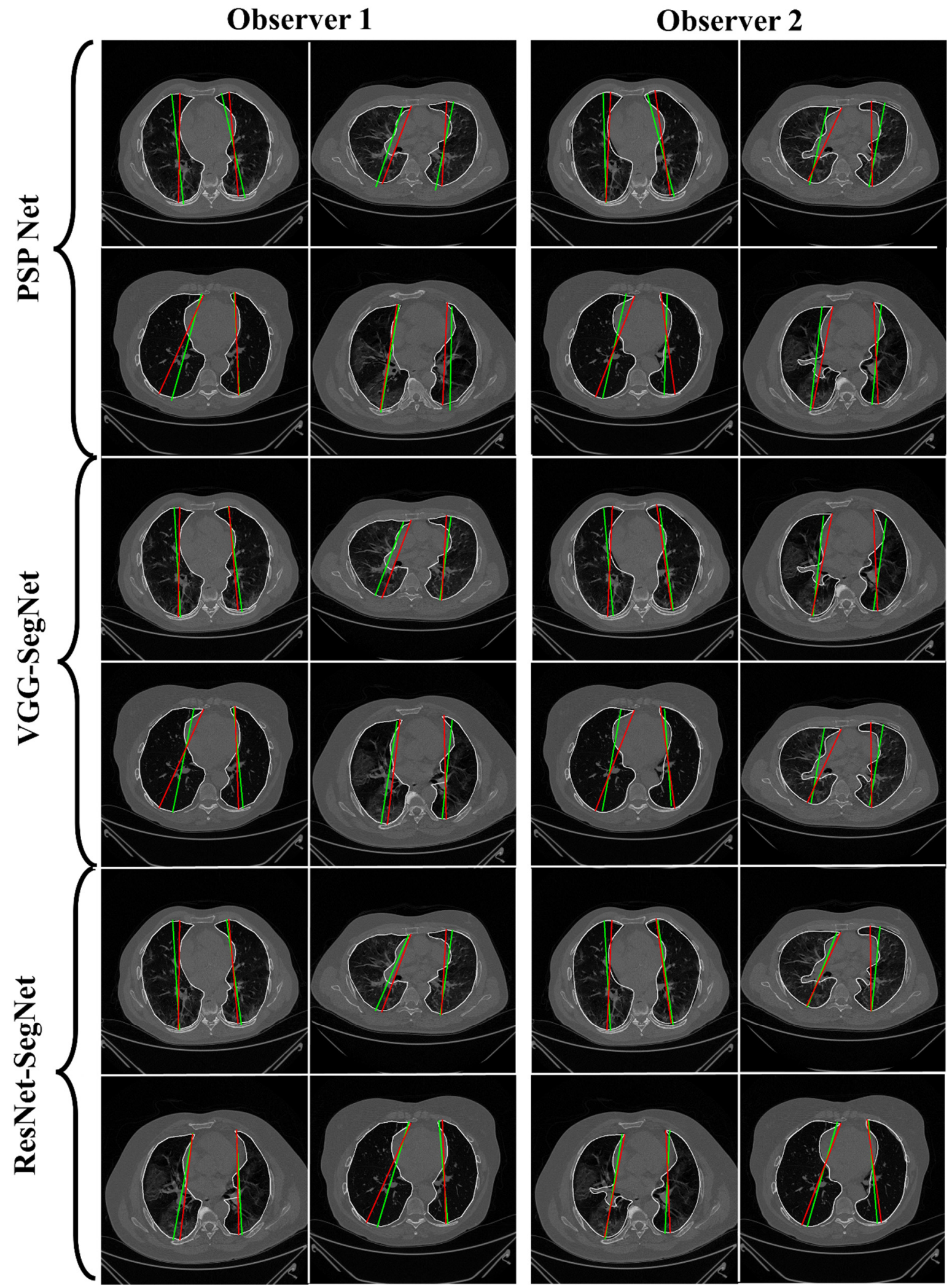

Figure 11. AI-model long axis (green) vs. GT long axis (red) for Observer 1 and Observer 2. 
Observer 1
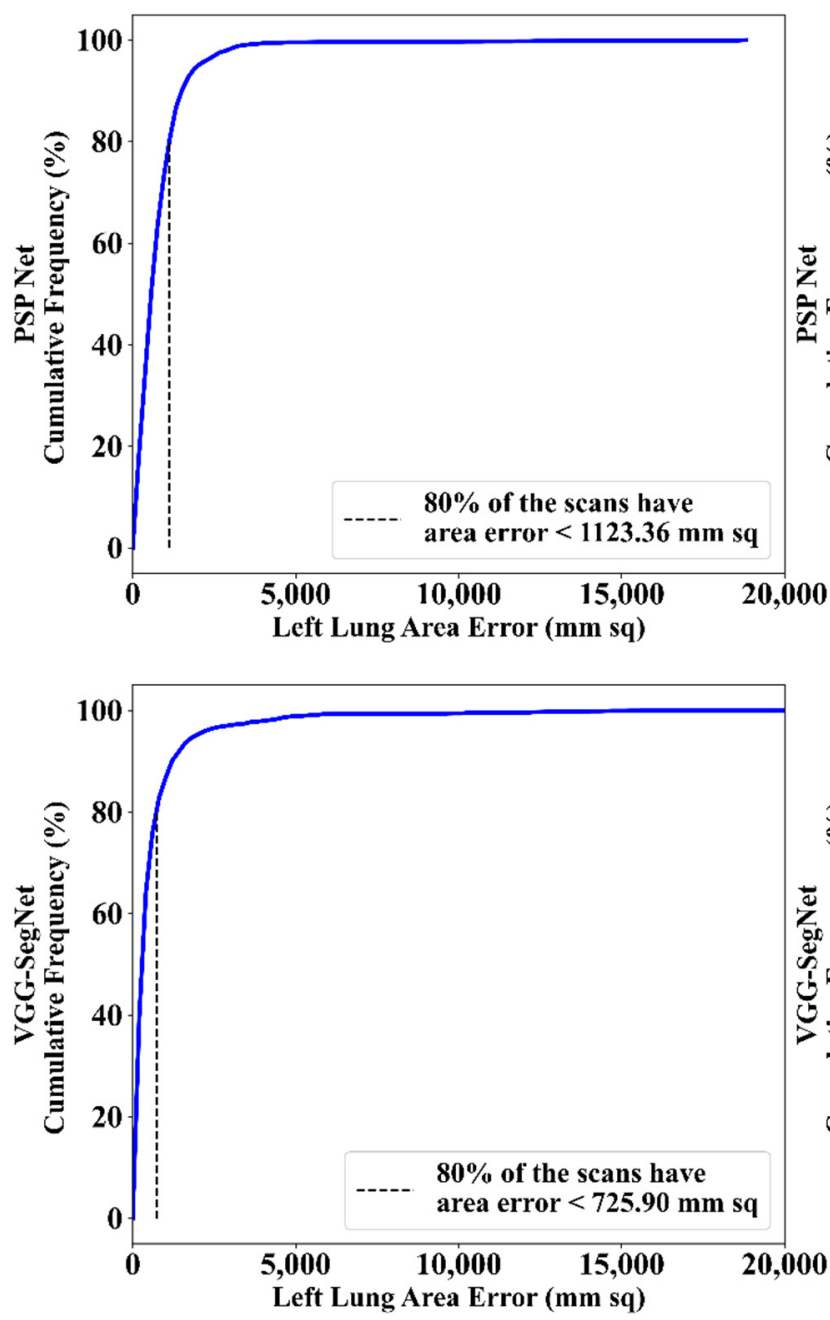

Observer 2
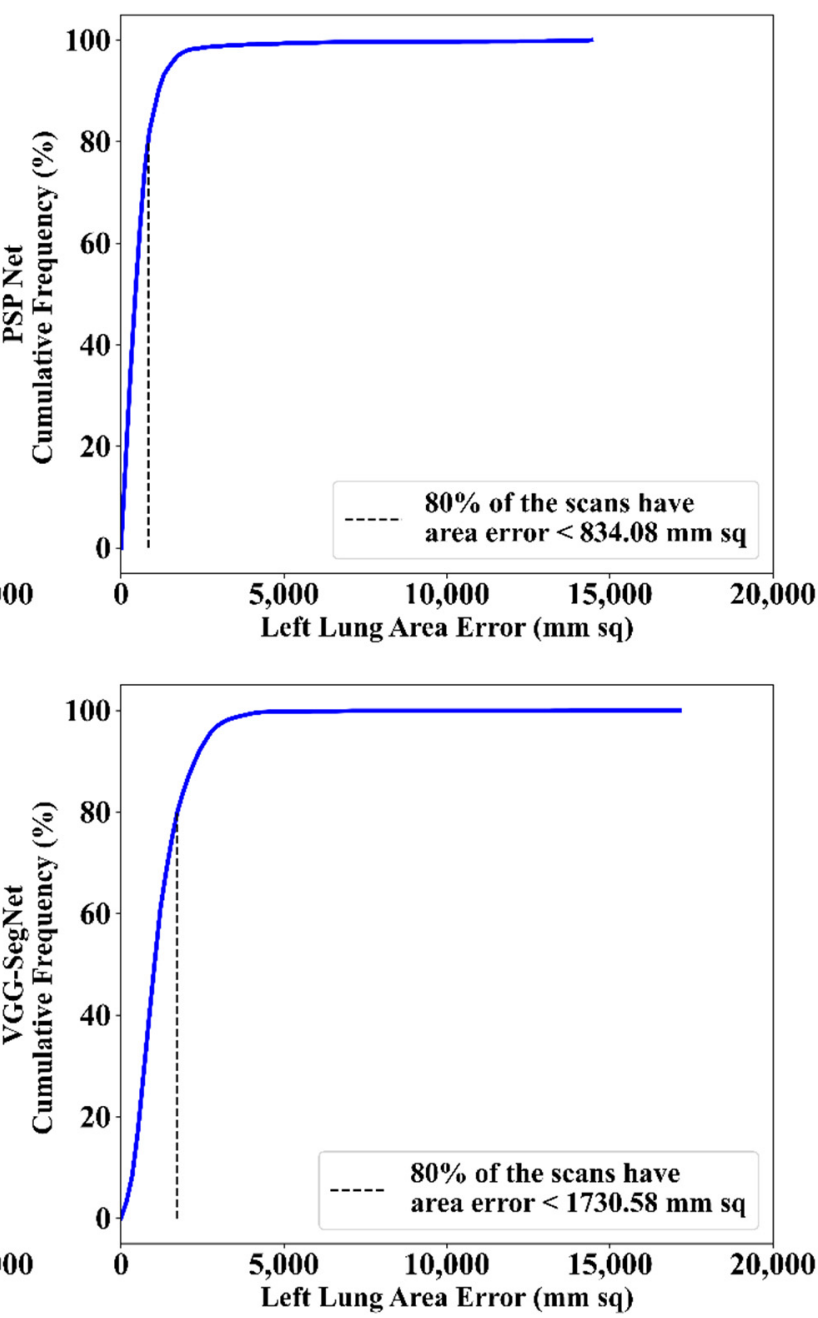
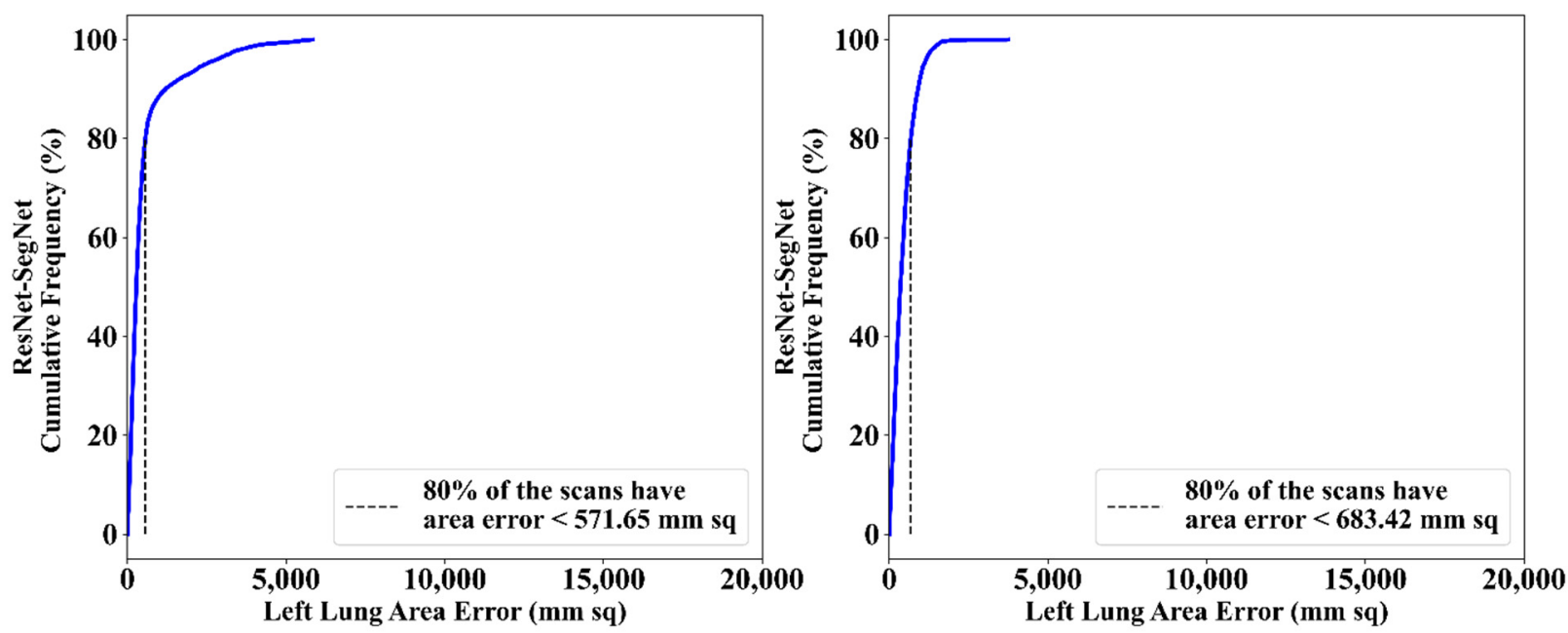

Figure 12. Cumulative frequency plot of left LAE using three AI models: Observer 1 vs. Observer 2. 
Observer 1

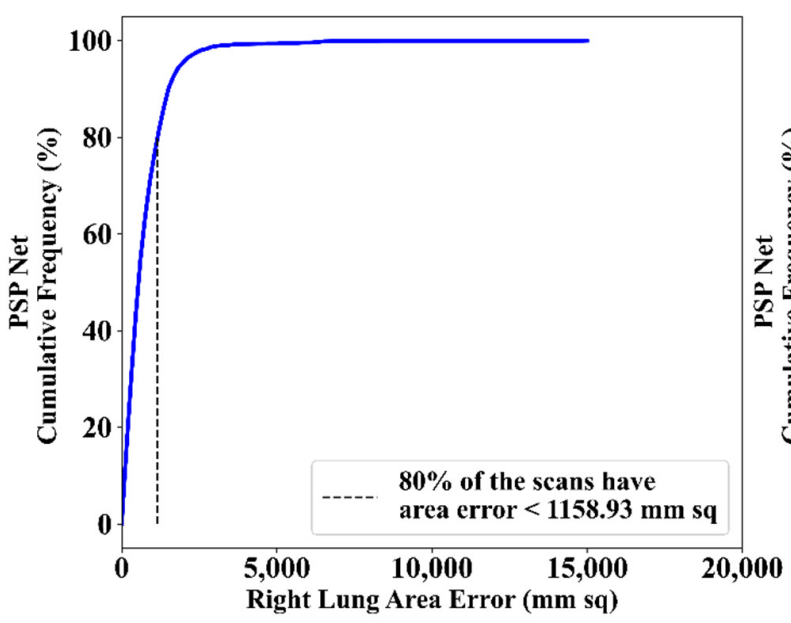

Observer 2

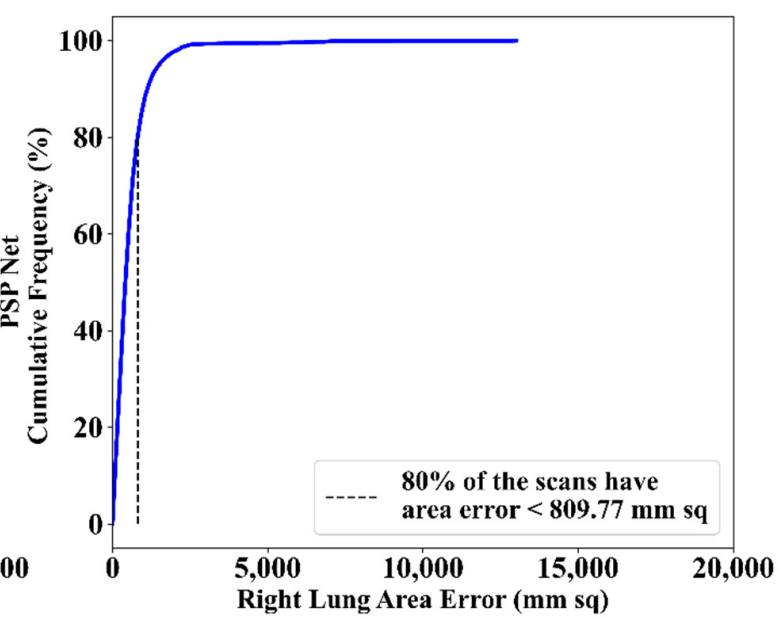

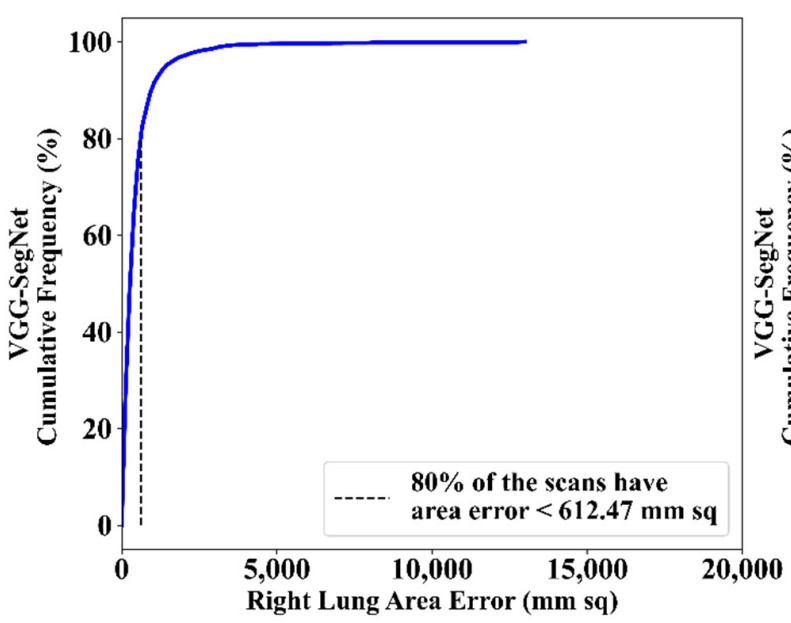
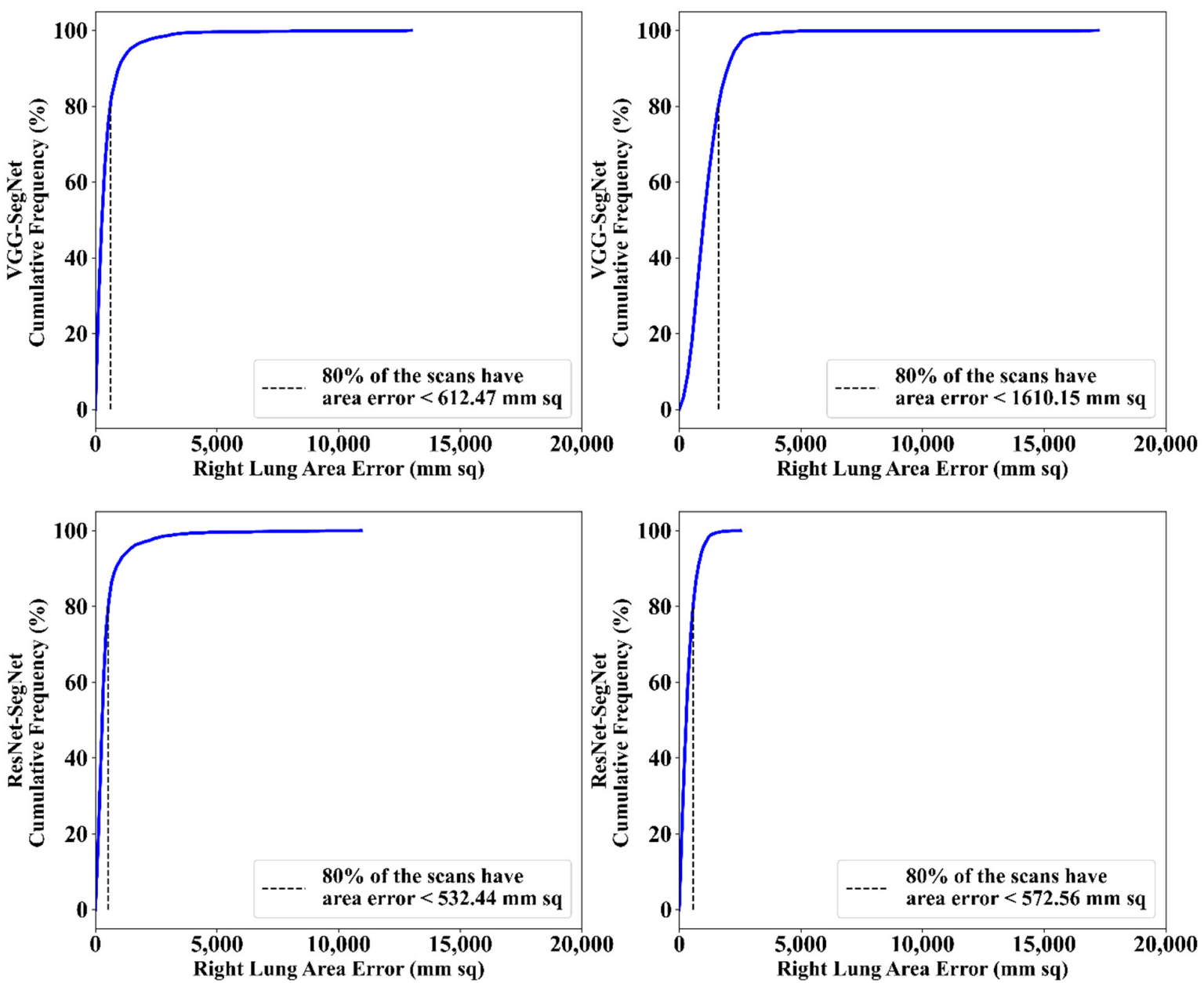

Figure 13. Cumulative frequency plot of right LAE using three AI models: Observer 1 vs. Observer 2.

Correlation Plot for Lung Area Error

Coefficient of correlations (CC) plots for the three AI models' LA vs. GT, area corresponding to the left and right between Observers 1 and 2, are shown in Figures 14 and 15. The CC values are summarized in Table 1 with a percentage difference between Observers 1 and 2 . The percentage difference for the CC value $(p<0.001)$ ranges from $0 \%$ to $2.04 \%$, which is $<5 \%$ as part of the error threshold chosen as the hypothesis. This clearly shows that the AI models are clinically valid for the proposed setting of the inter-observer variability study. 
Observer 1
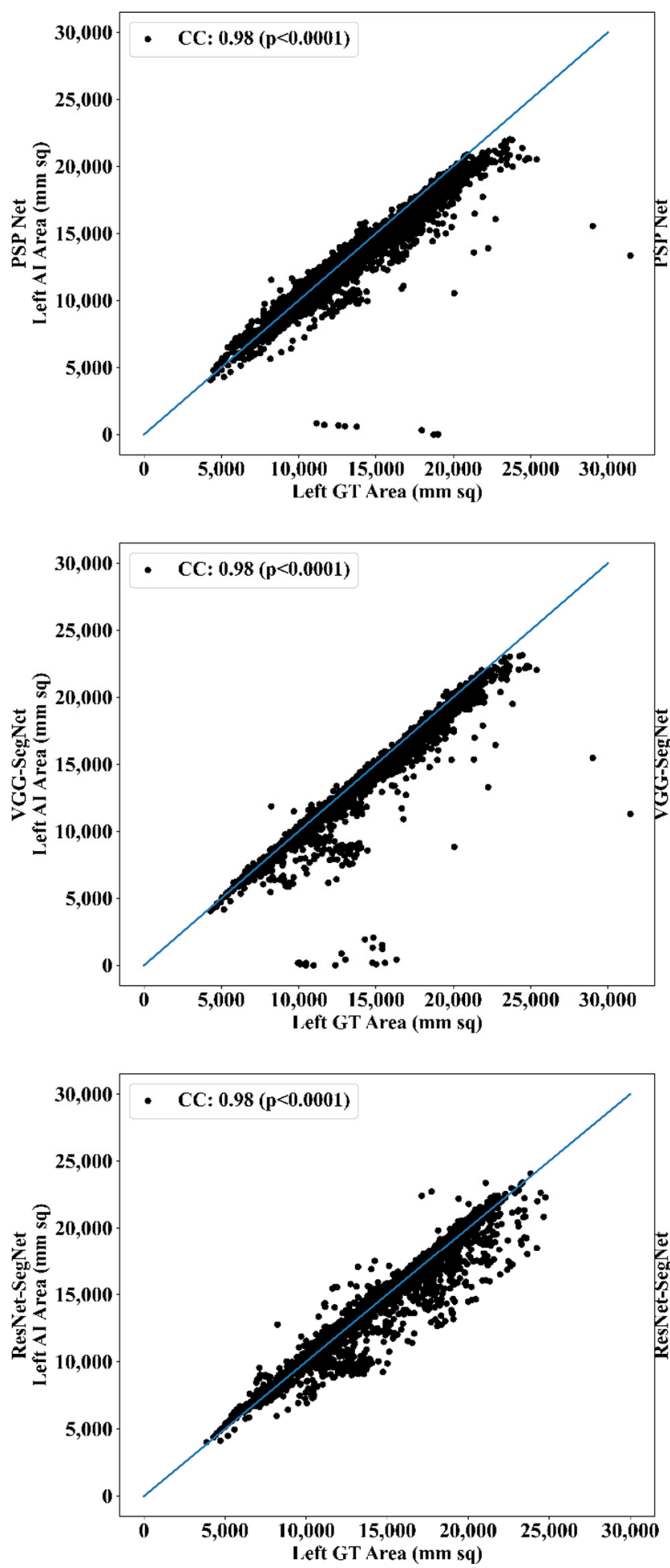

Observer 2
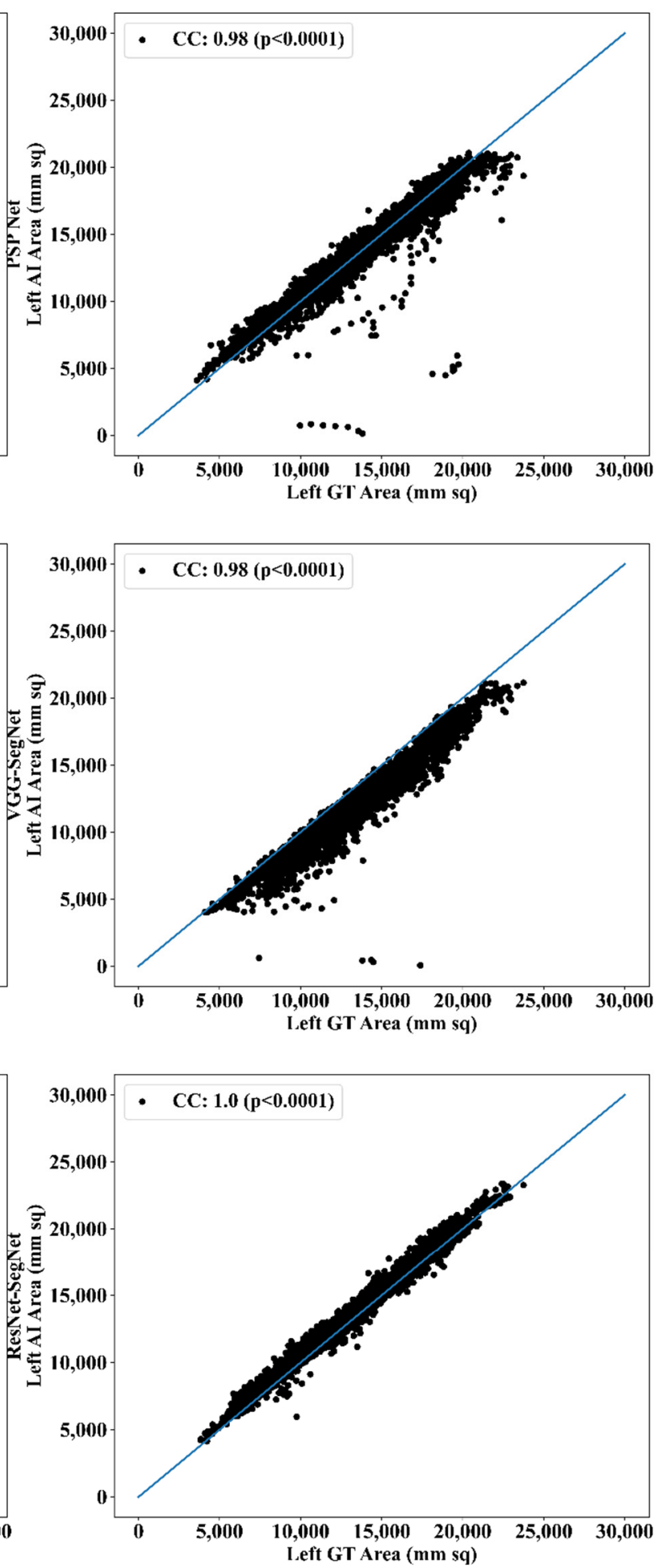

Figure 14. CC of left lung area using three AI models: Observer 1 vs. Observer 2. 
Observer 1
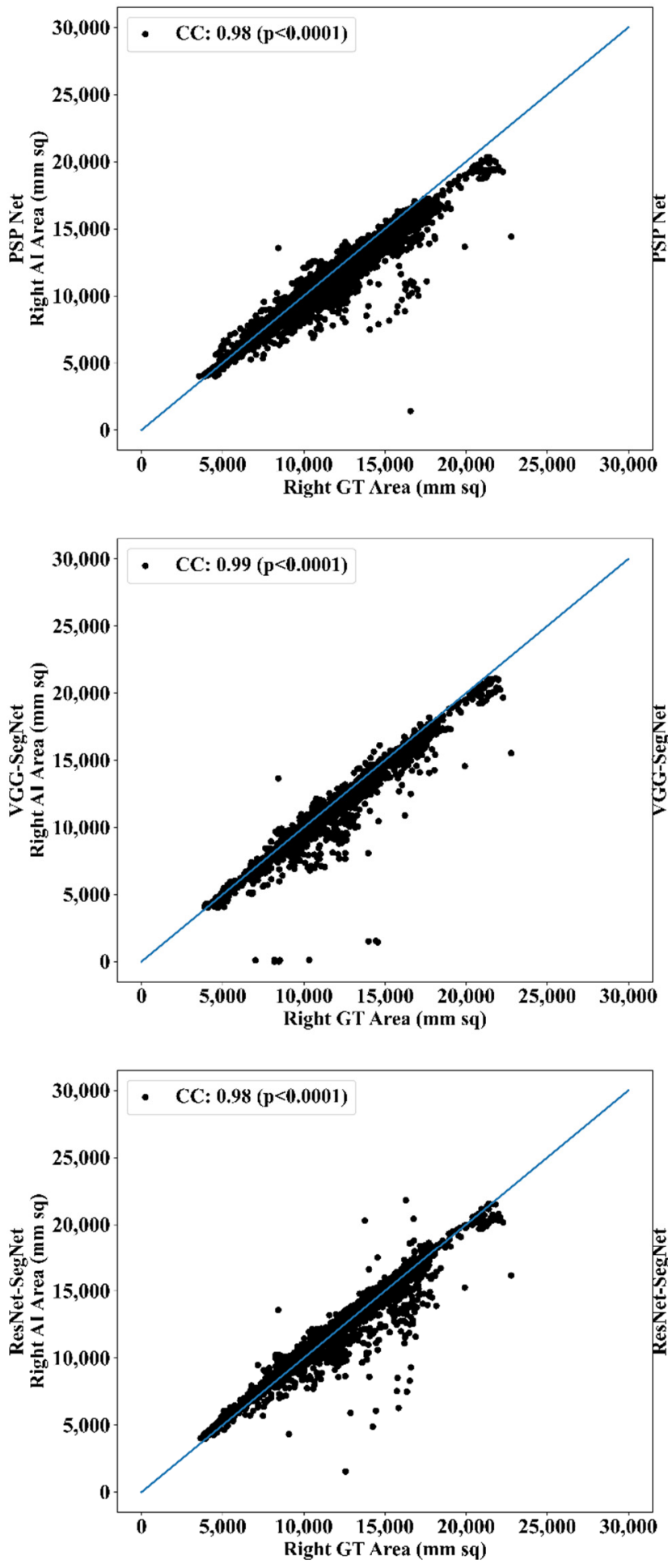

\section{Observer 2}
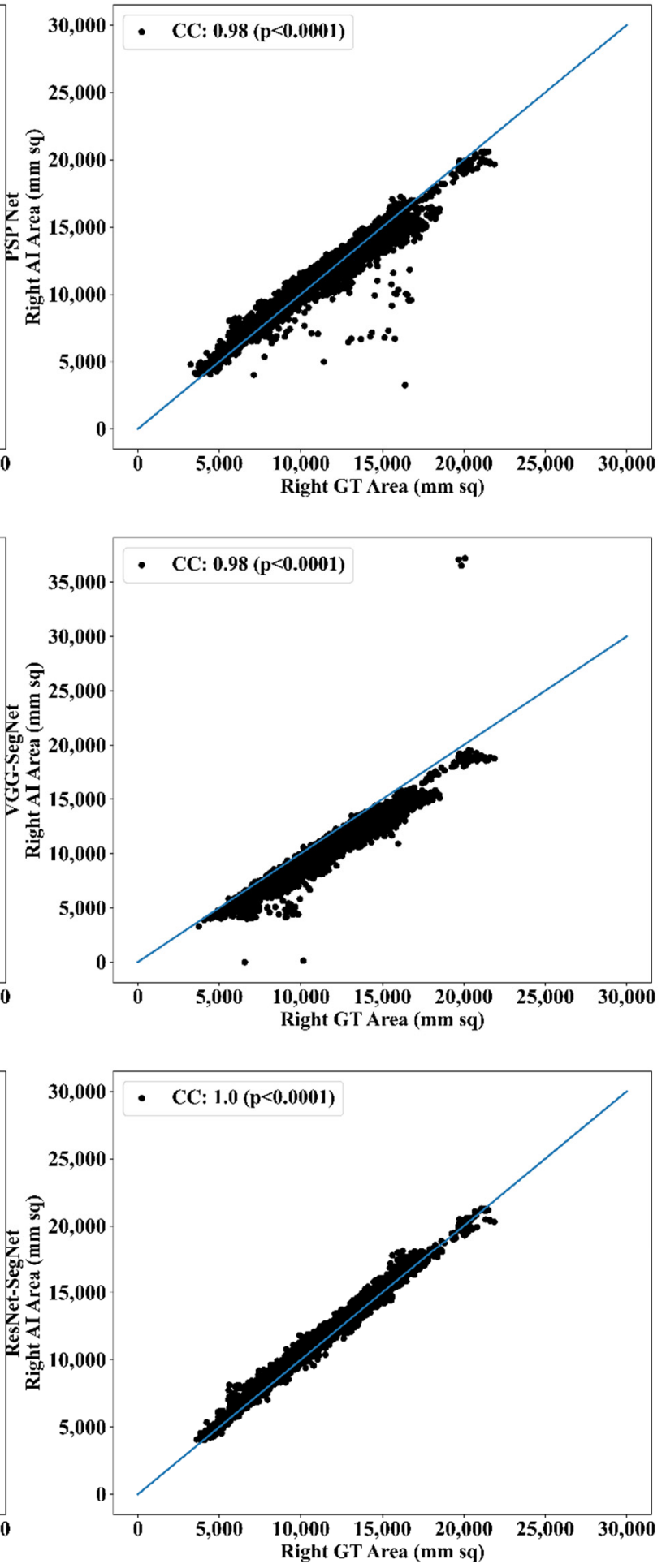

Figure 15. CC of right lung area using three AI models: Observer 1 vs. Observer 2.

Jaccard Index and Dice Similarity

Figure 16 depicts a cumulative frequency plot for dice similarity (DS) for three AI models between Observers 1 and Observer 2 . It shows that $80 \%$ of the CT images had a DS $>0.95$. A cumulative frequency plot for the Jaccard Index (JI) is presented in Figure 17 and shows that $80 \%$ of the CT scans had a JI > 0.90 between Observer 1 and Observer 2 . The three AI models follow the order: PSP Net, VGG-SegNet, and ResNet-SegNet. 
Table 1. Comparison of the CC values obtained between AI model area and the GT area corresponding to Observer 1 and Observer 2.

\begin{tabular}{ccccccccccc}
\hline & \multicolumn{3}{c}{ PSP Net } & \multicolumn{3}{c}{ VGG-SegNet } & \multicolumn{3}{c}{ ResNet-SegNet } \\
\cline { 2 - 10 } & Left & Right & Mean & Left & Right & Mean & Left & Right & Mean \\
\hline Observer 1 & 0.98 & 0.98 & 0.98 & 0.98 & 0.99 & 0.99 & 0.98 & 0.98 & 0.98 \\
\hline Observer 2 & 0.98 & 0.98 & 0.98 & 0.98 & 0.98 & 0.98 & 1.00 & 1.00 & 1.00 \\
\hline \% Difference & 0.00 & 0.00 & 0.00 & 0.00 & 1.01 & 0.51 & 2.04 & 2.04 & 2.04 \\
\hline
\end{tabular}

Observer 1
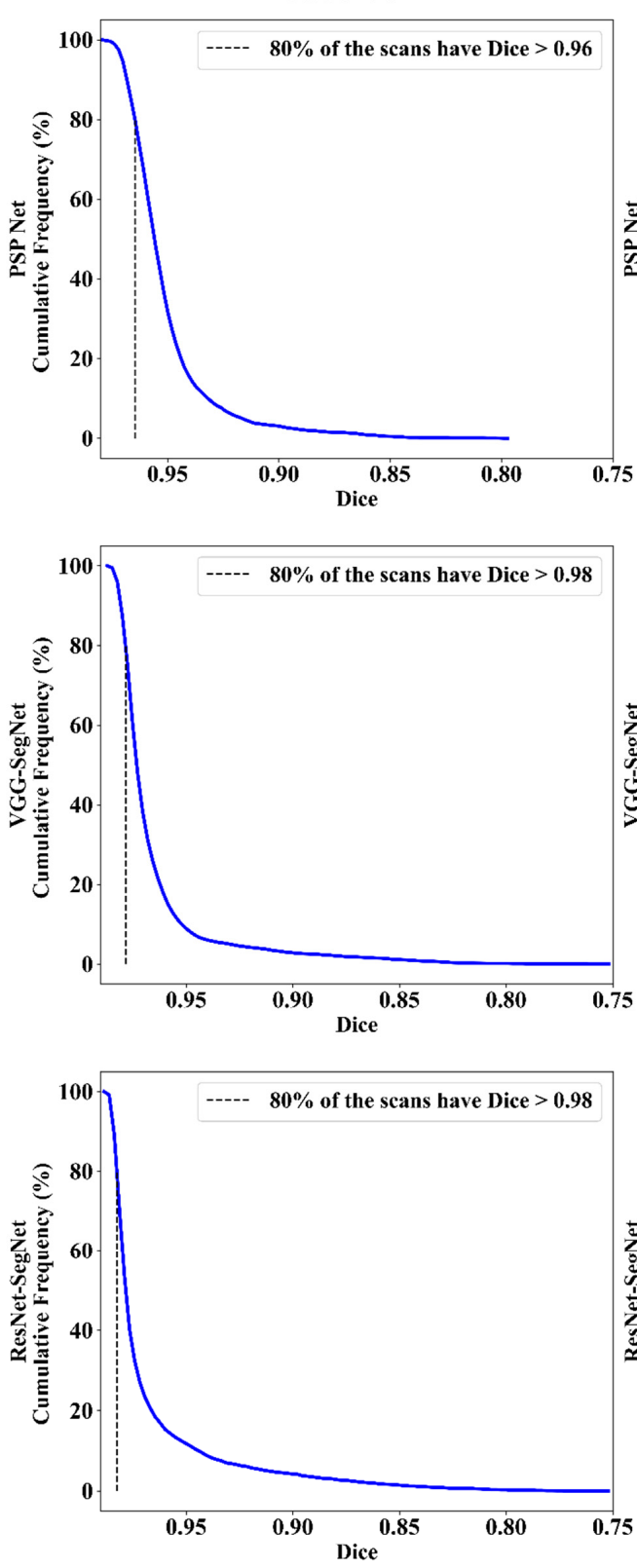

Observer 2
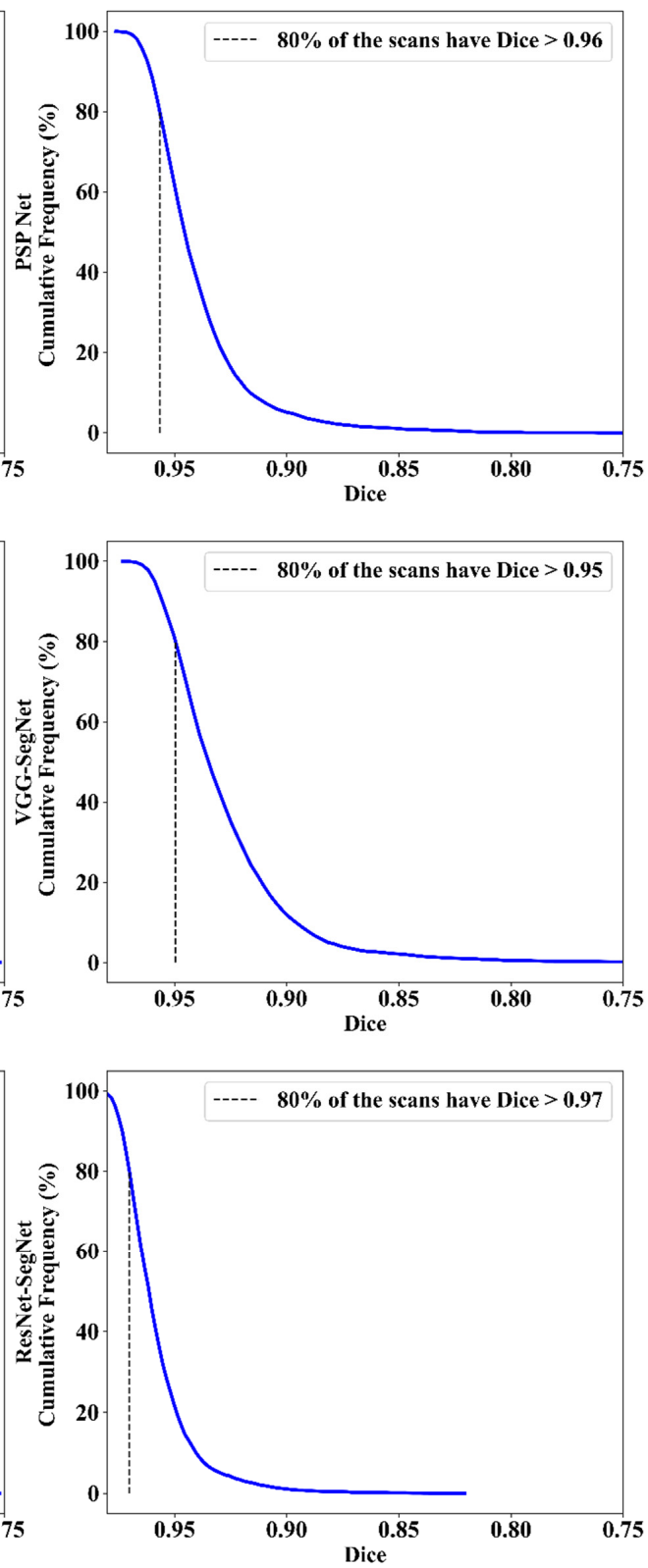

Figure 16. DS for combined lung using the three AI models: Observer 1 vs. Observer 2. 
Observer 1
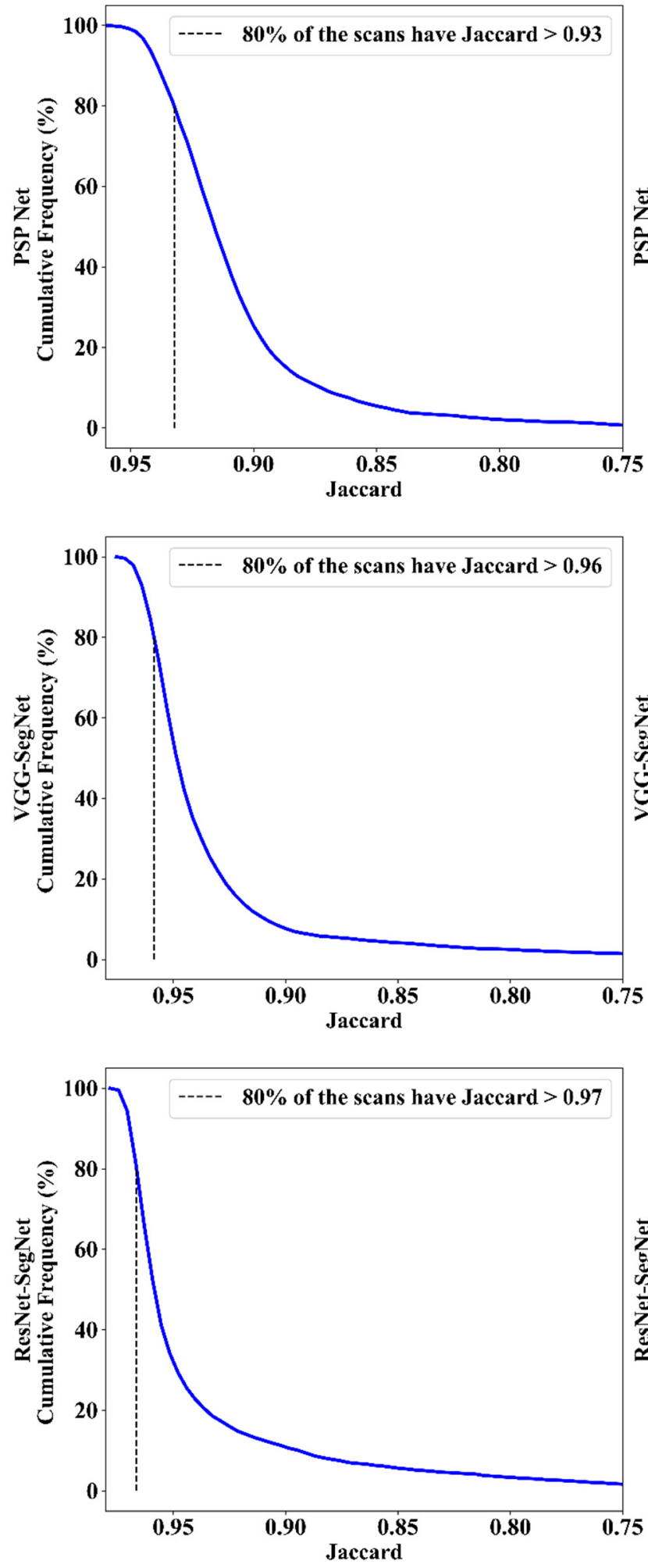

Observer 2
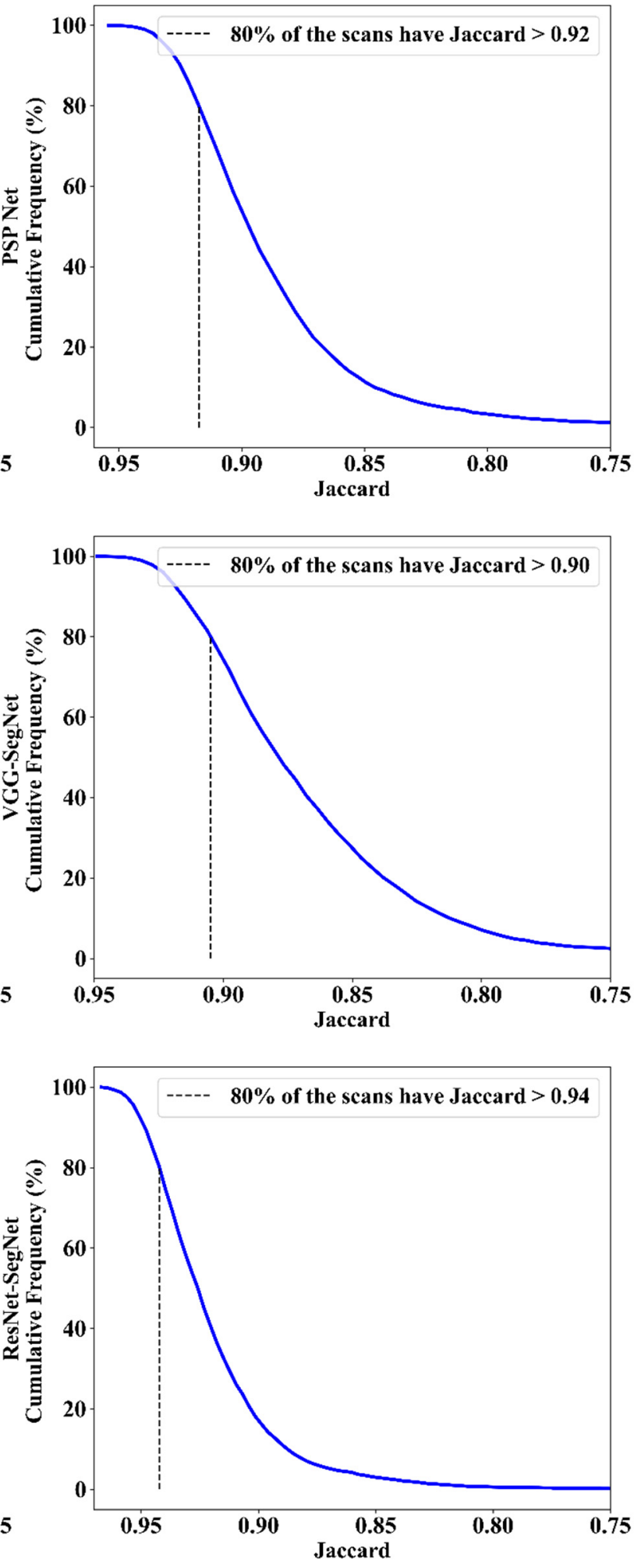

Figure 17. JI for combined lung using three AI models: Observer 1 vs. Observer 2. Bland-Altman Plot for Lung Area

A Bland-Altman plot is used to demonstrate the consistency of two methods that employ the same variable. Based on our prior paradigms [48,62], we follow the Bland-Altman 
computing procedure. Figures 18 and 19 show the (i) mean and (ii) standard deviation of the lung area between the AI model and GT area corresponding to Observers 1 and Observer 2.

\section{Observer 1}
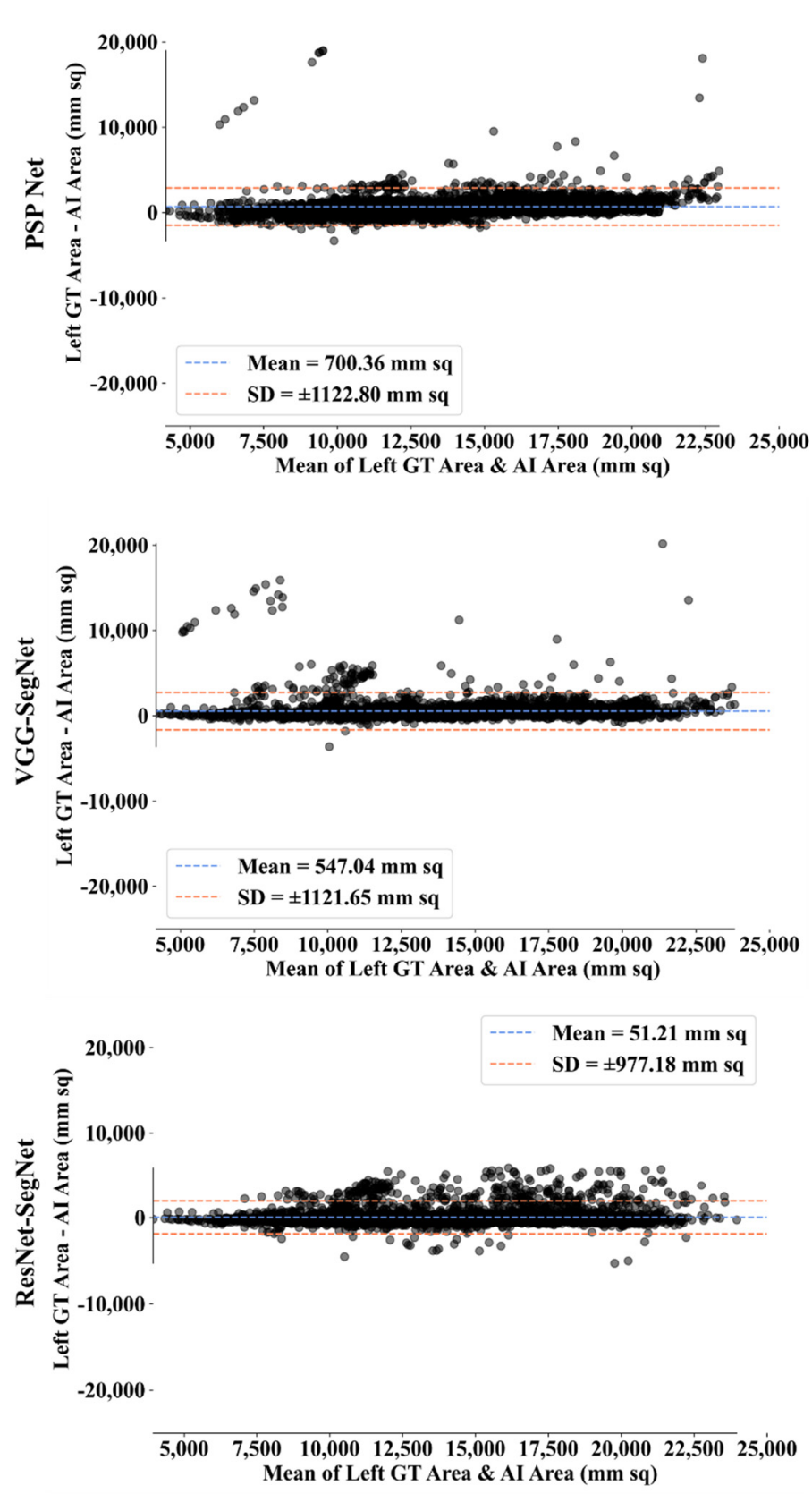

Observer 2
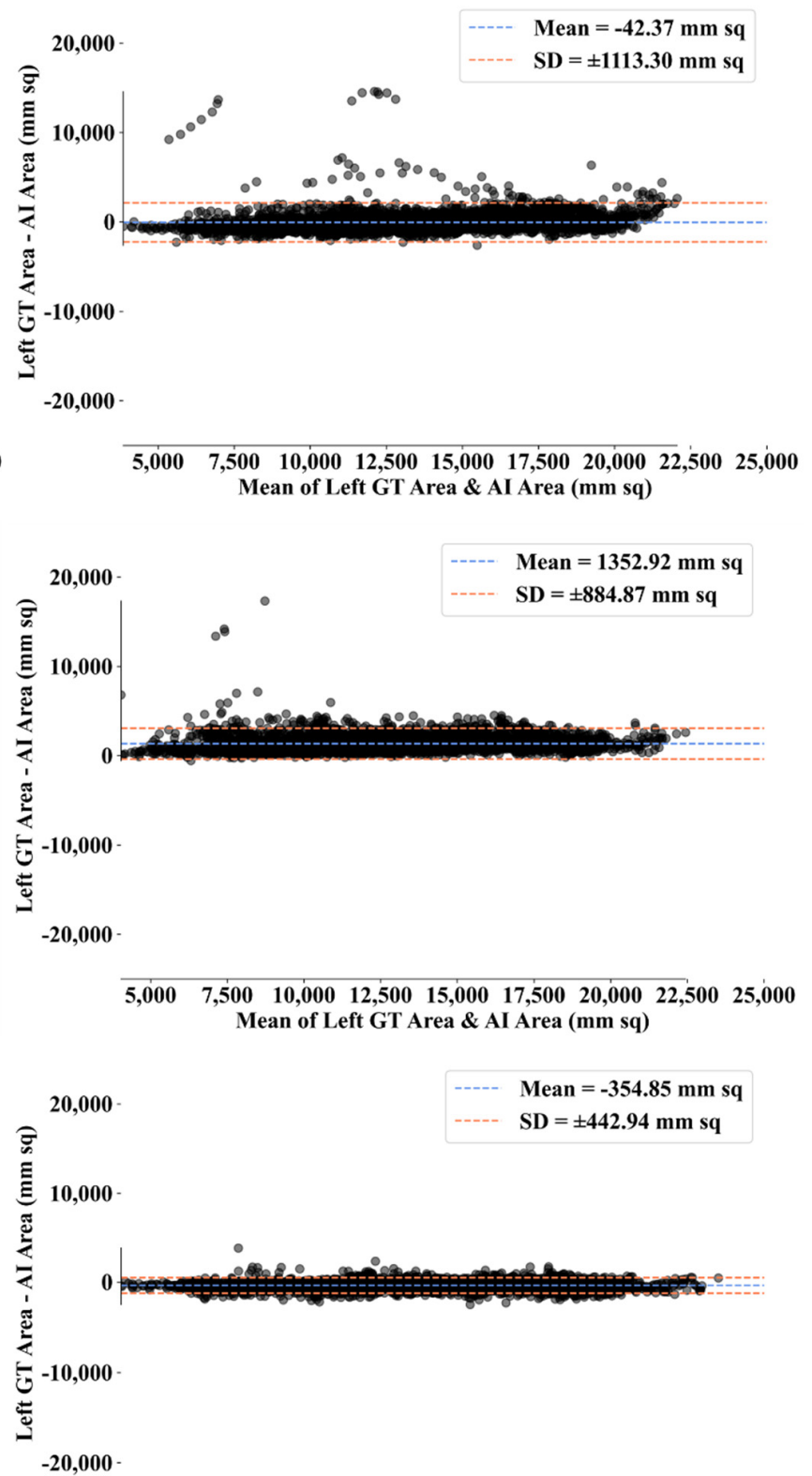

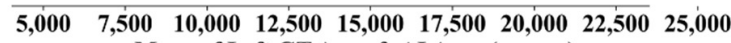
Mean of Left GT Area \& AI Area (mm sq)

Figure 18. BA for left LA for three AI models: Observer 1 vs. Observer 2. 


\section{Observer 1}

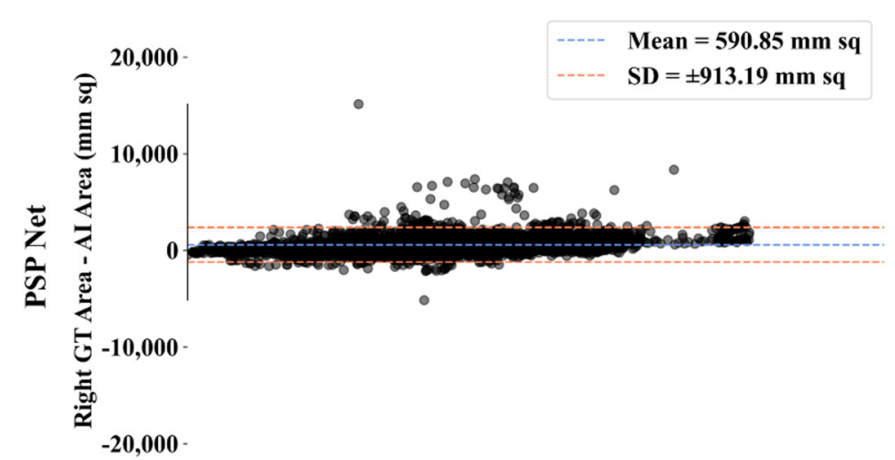

$\begin{array}{lllllllll}5,000 & 7,500 & 10,000 & 12,500 & 15,000 & 17,500 & 20,000 & 22,500 & 25,000\end{array}$ Mean of Right GT Area \& AI Area (mm sq)

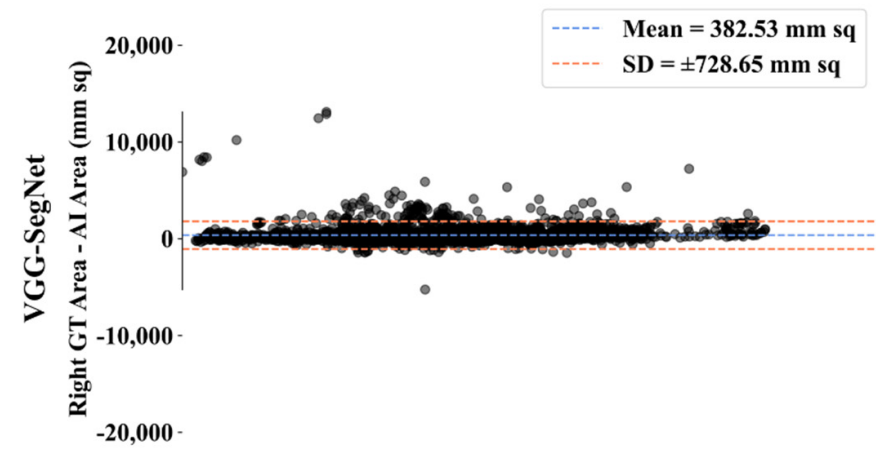

$\begin{array}{lllllllll}5,000 & 7,500 & 10,000 & 12,500 & 15,000 & 17,500 & 20,000 & 22,500 & 25,000\end{array}$ Mean of Right GT Area \& AI Area (mm sq)

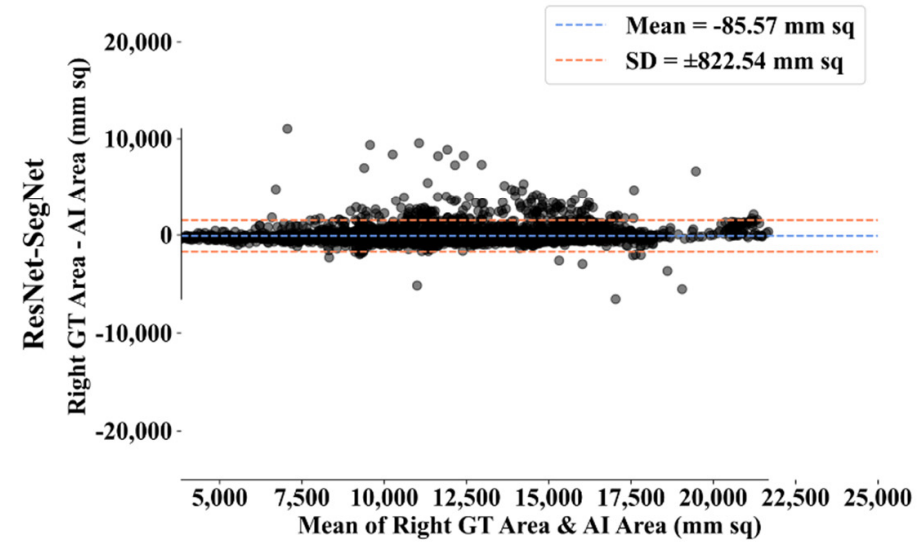

Observer 2
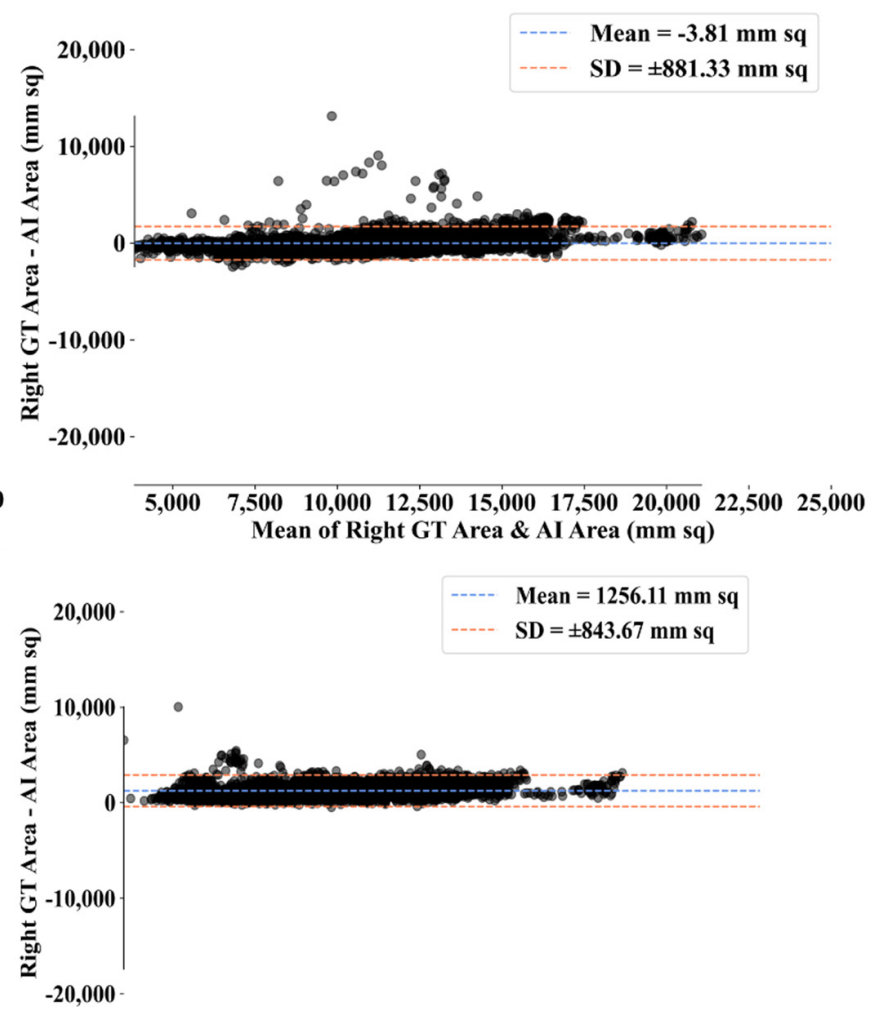

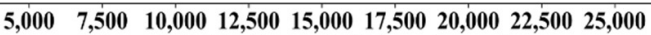
Mean of Right GT Area \& AI Area (mm sq)

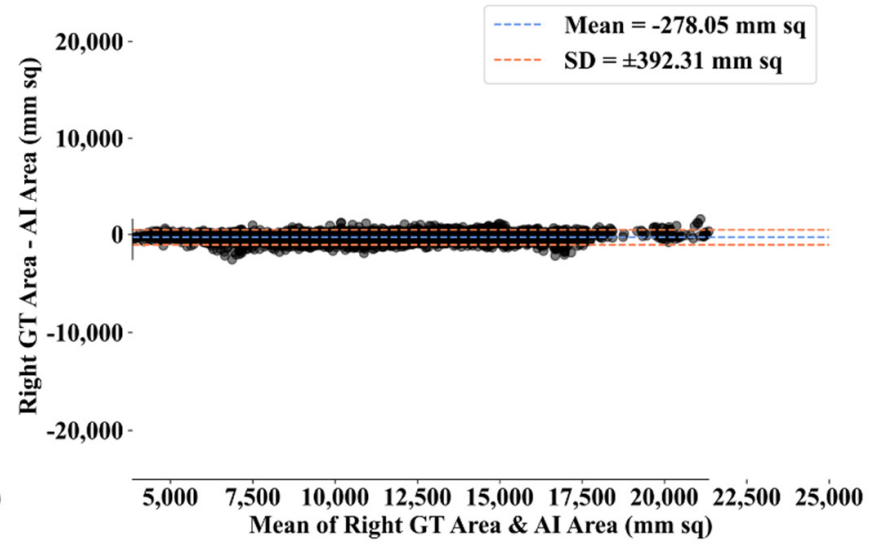

Figure 19. BA for right LA using three AI models: Observer 1 vs. Observer 2.

ROC Plots for Lung Area

An ROC curve represents how an AI system's diagnostic performance changes as the discrimination threshold changes. Figure 20 shows the ROC curve and corresponding AUC value for the three AI models between Observer 1 and Observer 2. The three AI models follow the order: PSP Net, VGG-SegNet, and ResNet-SegNet. 

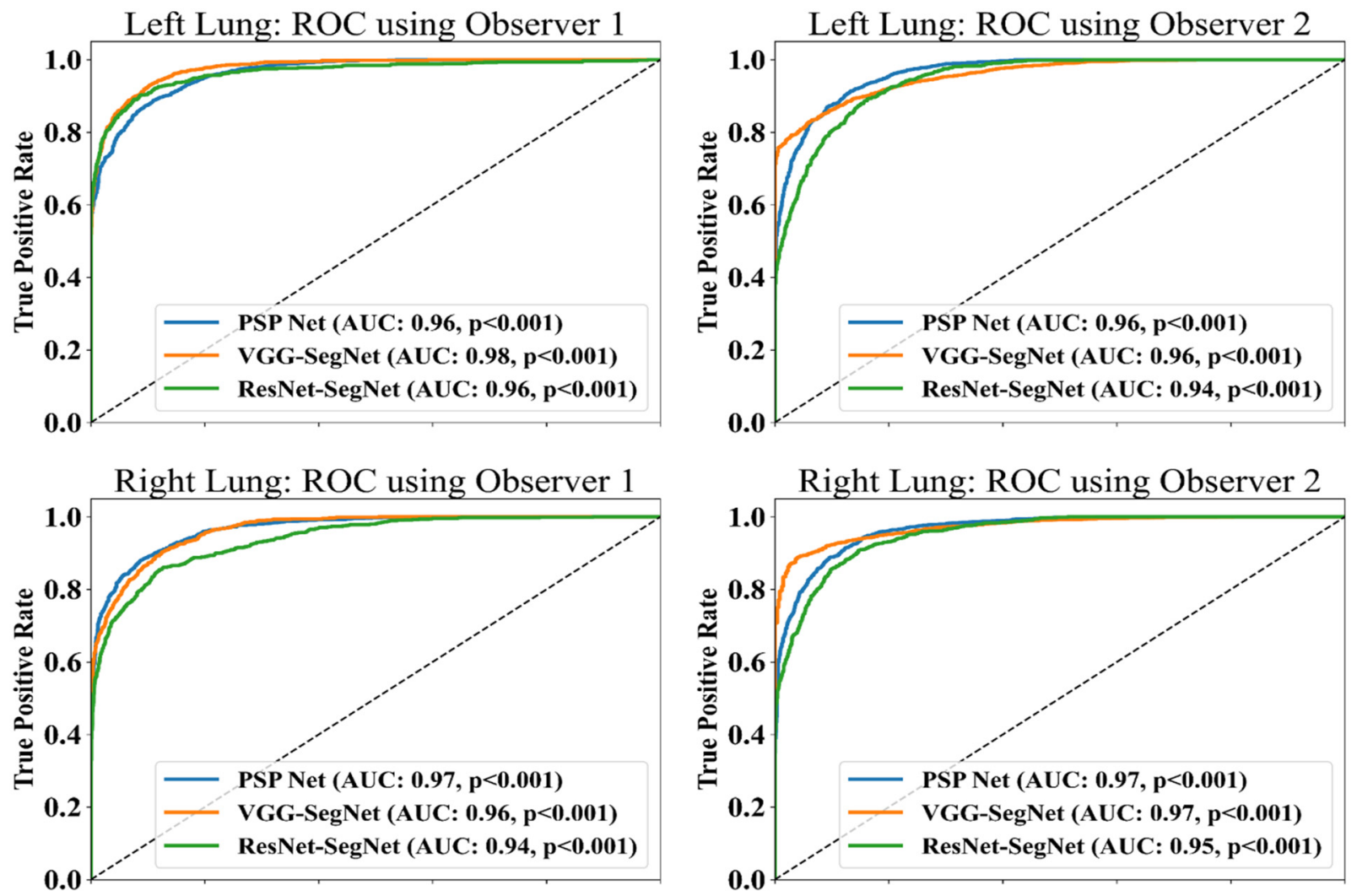

Combined Lung: ROC using Observer 1

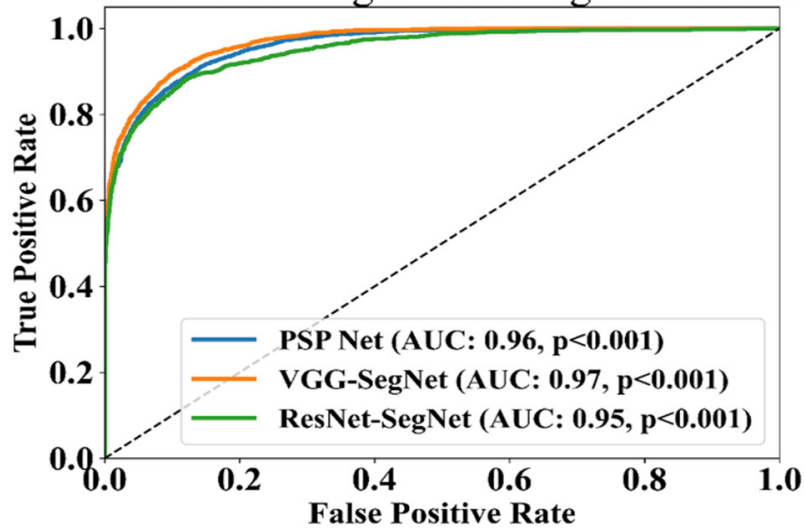

Combined Lung: ROC using Observer 2

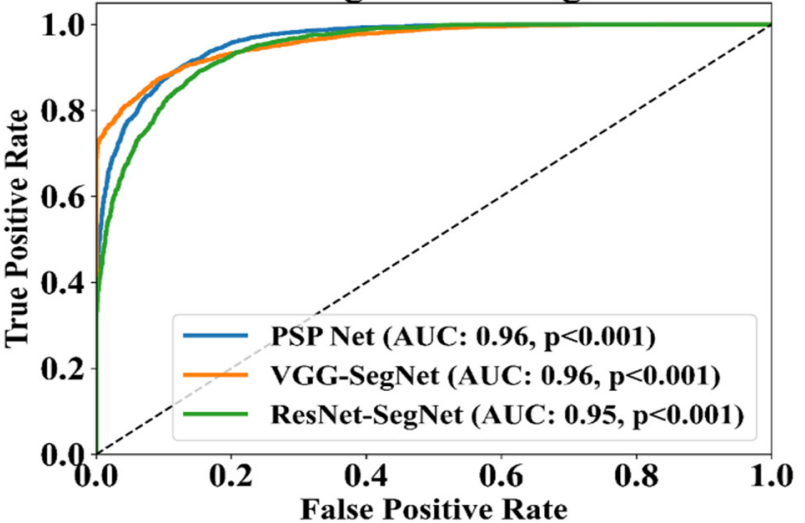

Figure 20. ROC and AUC curve for the three AI models: Observer 1 vs. Observer 2.

\subsubsection{Performance Evaluation Using Lung Long Axis Error}

Cumulative Frequency Plot for Lung Long Axis Error

Figures 21 and 22 show the cumulative frequency plot LLAE for left and right lung, respectively, corresponding to Observer 1 and Observer 2 for the three AI models. Based on the $80 \%$ threshold, the LLAE for the left lung (Figure 21) using the three AI models for Observer 1 and Observer 2 were $6.12 \mathrm{~mm}$ (for PSP Net), $4.77 \mathrm{~mm}$ (for VGG-SegNet), and $5.01 \mathrm{~mm}$ (for ResNet-SegNet) and $10.88 \mathrm{~mm}$ (for PSP Net), $13.30 \mathrm{~mm}$ (for VGG-SegNet), and $9.18 \mathrm{~mm}$ (for ResNet-SegNet), respectively. Similarly, for the right lung (Figure 22), the error was $7.81 \mathrm{~mm}$ (for PSP Net), $5.47 \mathrm{~mm}$ (for VGG-SegNet), and $3.10 \mathrm{~mm}$ (for ResNet-SegNet) and $9.14 \mathrm{~mm}$ (for PSP Net), $11.33 \mathrm{~mm}$ (for VGG-SegNet), and $6.88 \mathrm{~mm}$ (for ResNet-SegNet), respectively, for Observer 1 and Observer 2. The three AI models follow the order: PSP Net, VGG-SegNet, and ResNet-SegNet. 
Observer 1
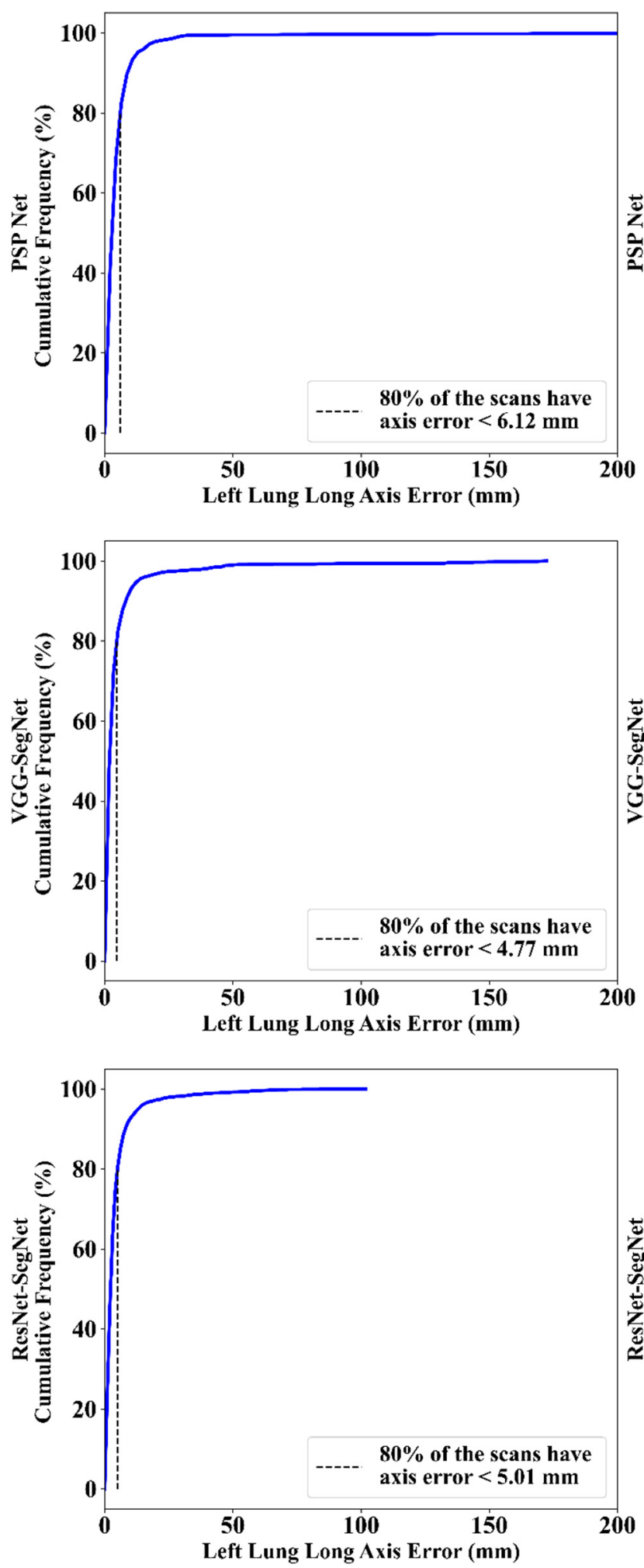

Observer 2
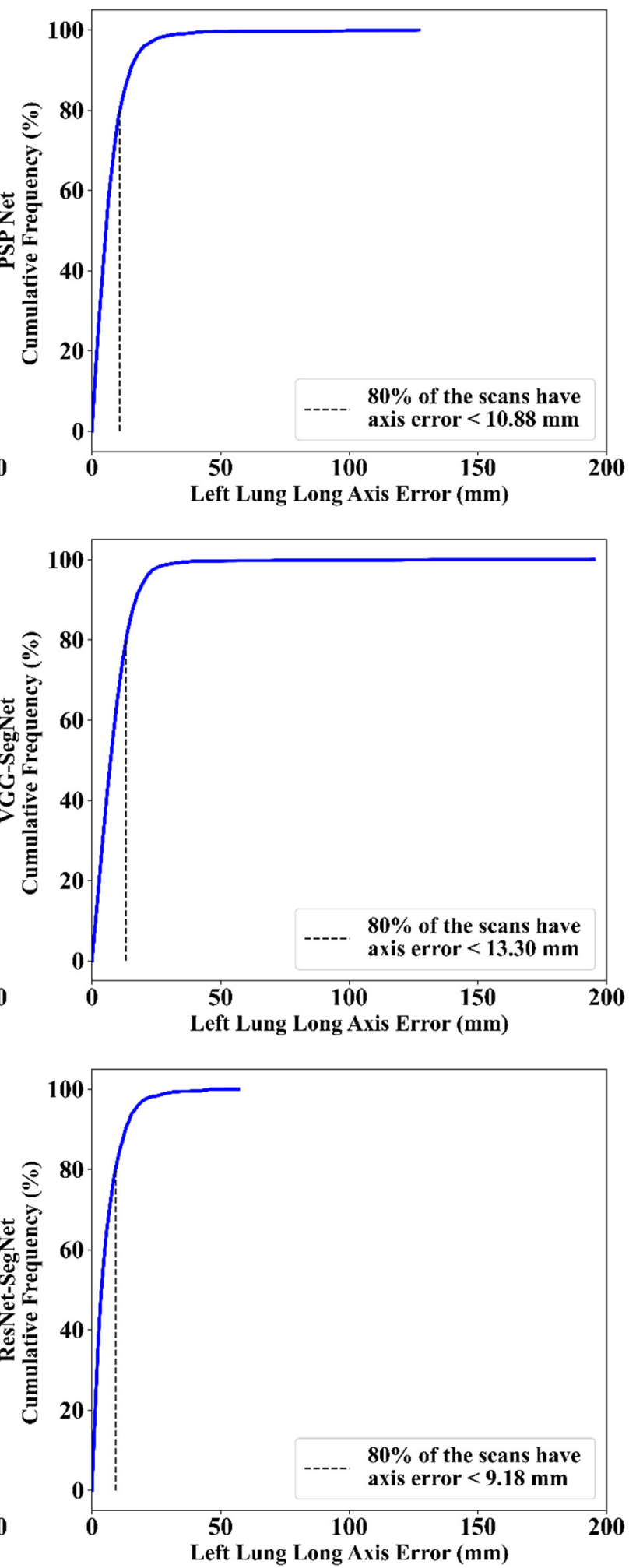

Figure 21. Cumulative frequency plot for left LLAE using three AI models: Observer 1 vs. Observer 2. 
Observer 1
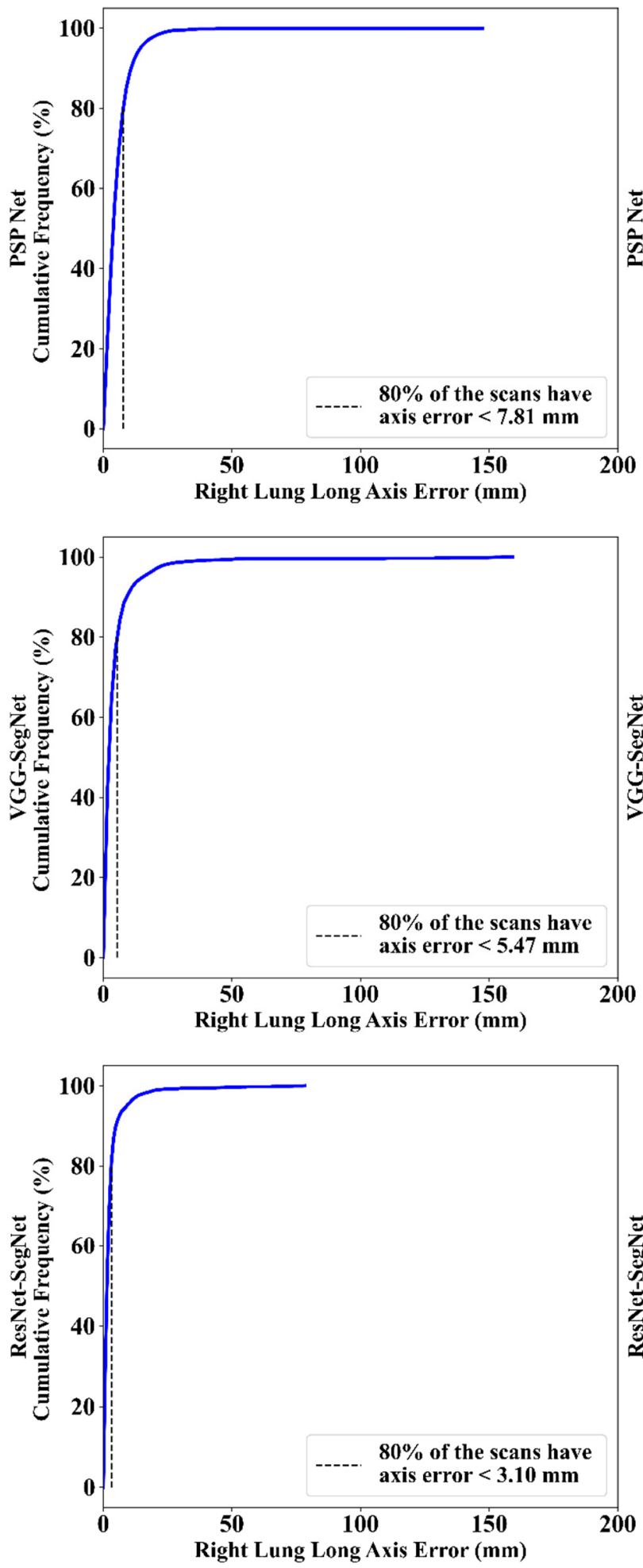

Observer 2
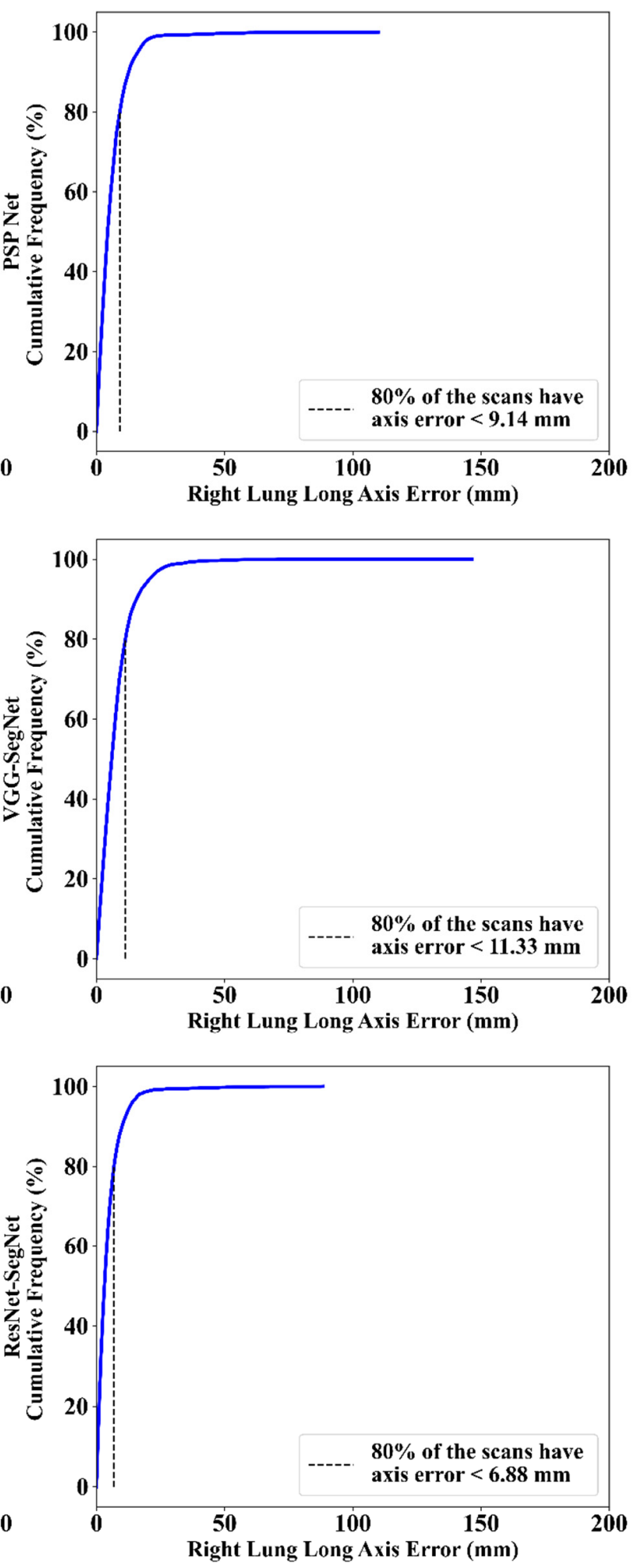

Figure 22. Cumulative frequency plot for right LLAE using three AI models: Observer 1 vs. Observer 2.

Correlation Plot for Lung Long Axis Error

Figures 23 and 24 show the CC plot for the three AI models considered in the proposed inter-observer variability study for Observers 1 and 2. Table 2 summarizes the CC values for the left, right, and mean errors of the LLA. It proves the hypothesis that the percentage difference between the results using the two observers has a difference of $<5 \%$. This demon- 
strates that the proposed system is clinically valid in the suggested inter-observer variability study context.

\section{Observer 1}
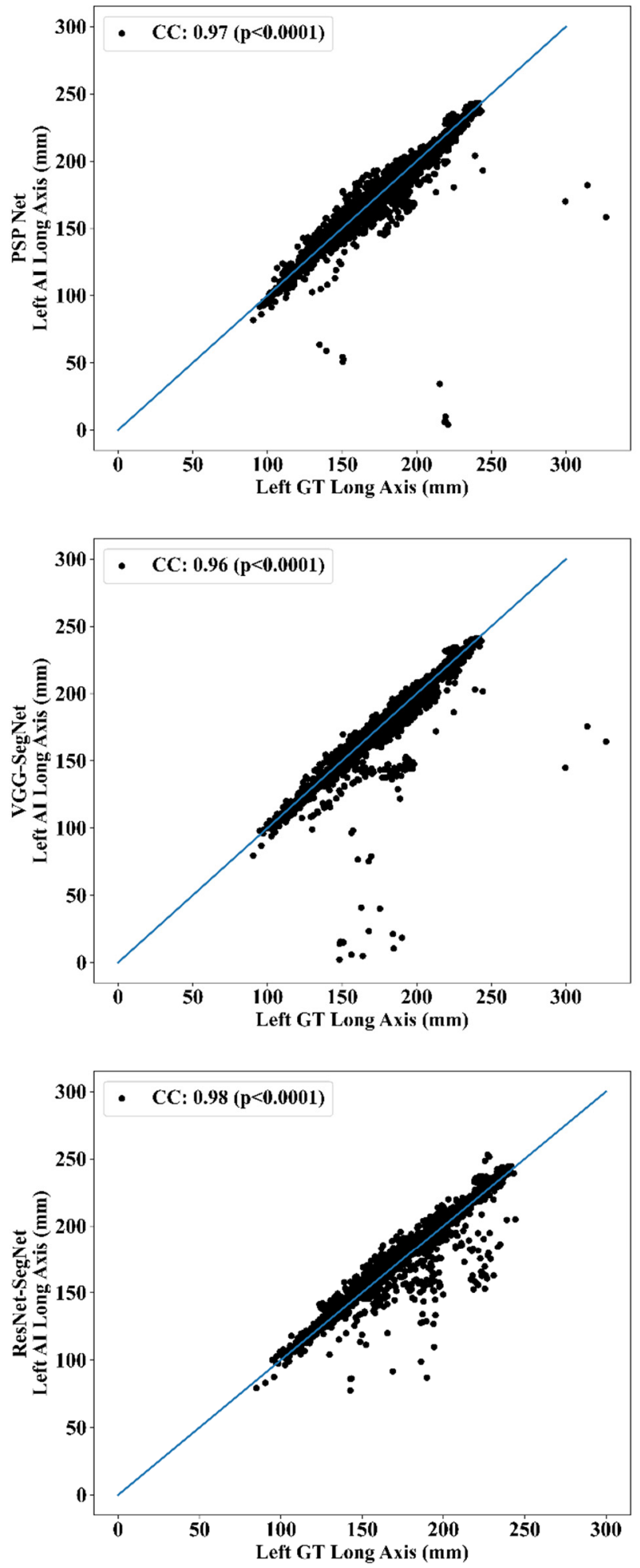

\section{Observer 2}
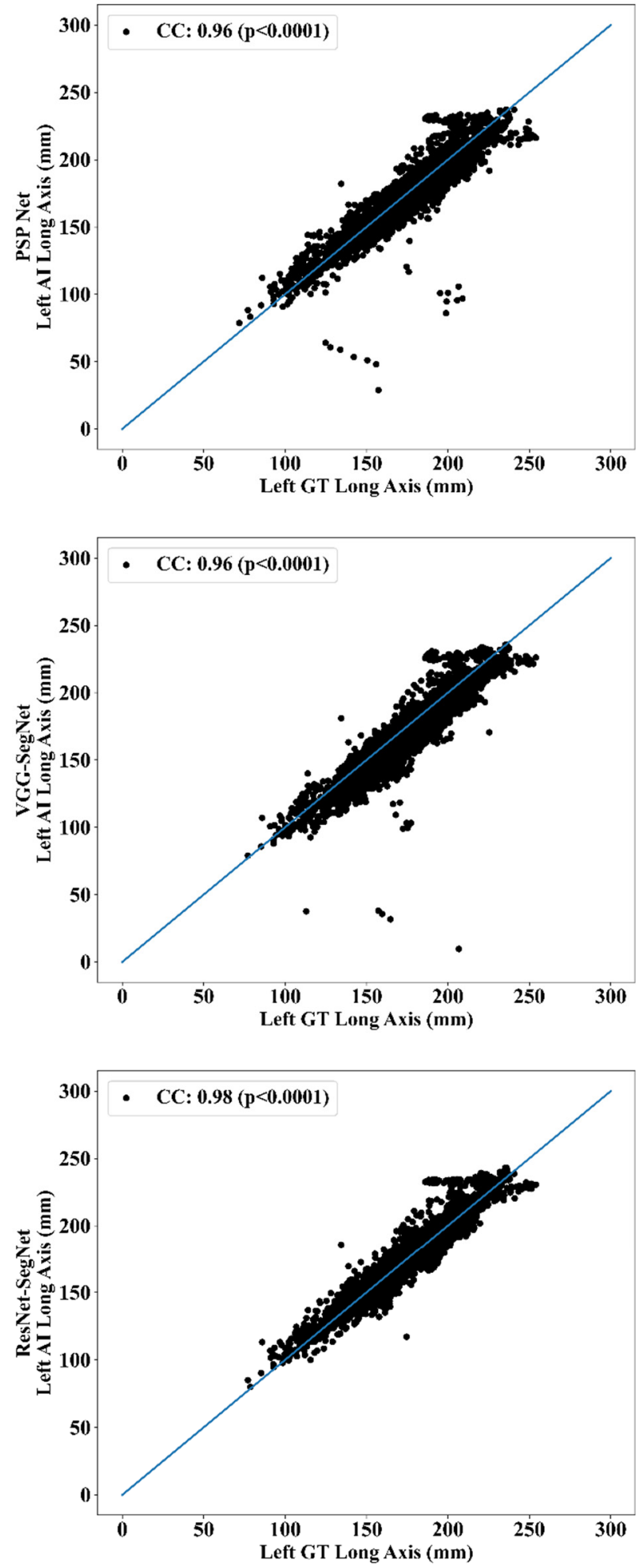

Figure 23. CC of left LLA for three AI models: Observer 1 vs. Observer 2. 


\section{Observer 1}
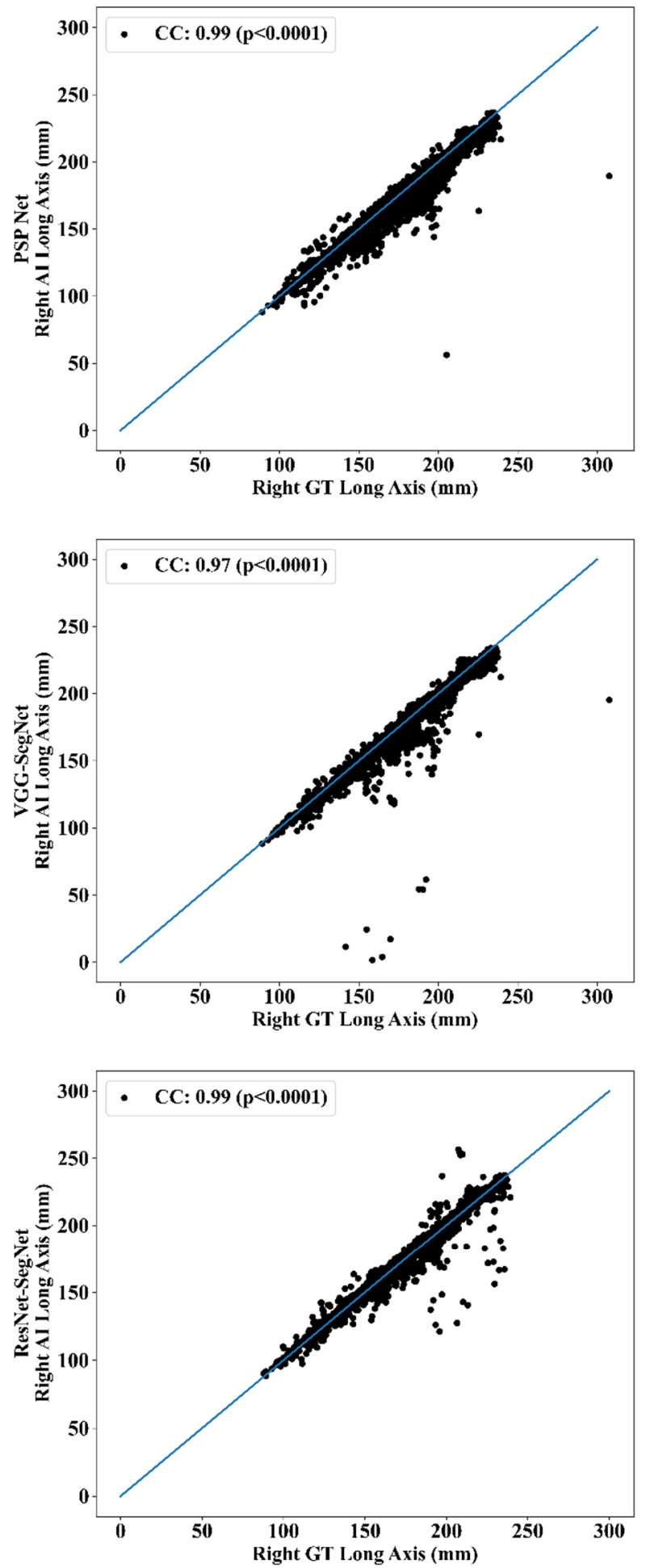

Observer 2
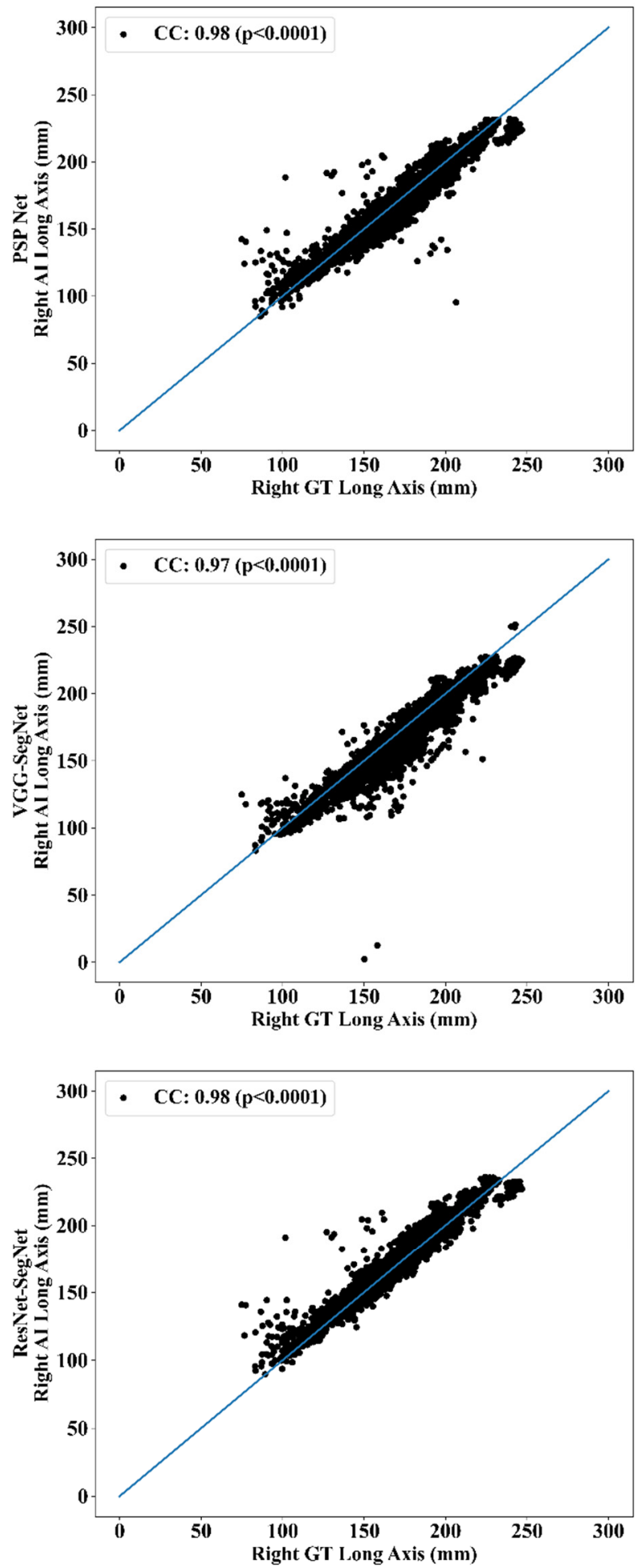

Figure 24. CC of right LLA using three AI models: Observer 1 vs. Observer 2.

Bland-Altman Plots for Lung Long Axis Error

The (i) mean and (ii) standard deviation of the lung long axis corresponding to Observer 1 and Observer 2 for the three AI models is shown in Figure 25 for the left lung and Figure 26 for the right lung. 
Table 2. Comparison of the CC values obtained between AI model lung long axis and the GT lung long axis corresponding to Observer 1 and Observer 2.

\begin{tabular}{ccccccccccc}
\hline & \multicolumn{3}{c}{ PSP Net } & \multicolumn{3}{c}{ VGG-SegNet } & \multicolumn{3}{c}{ ResNet-SegNet } \\
\cline { 2 - 10 } & Left & Right & Mean & Left & Right & Mean & Left & Right & Mean \\
\hline Observer 1 & 0.97 & 0.99 & 0.98 & 0.96 & 0.97 & 0.97 & 0.98 & 0.99 & 0.99 \\
\hline Observer 2 & 0.96 & 0.98 & 0.97 & 0.96 & 0.97 & 0.97 & 0.98 & 0.98 & 0.98 \\
\hline \% Difference & 1.03 & 1.01 & 1.02 & 0.00 & 0.00 & 0.00 & 0.00 & 1.01 & 0.51 \\
\hline
\end{tabular}

Observer 1

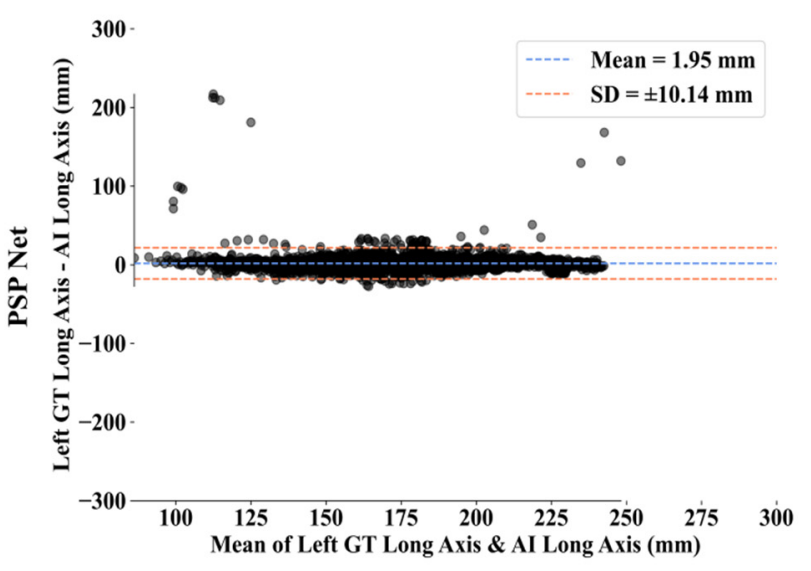

Observer 2
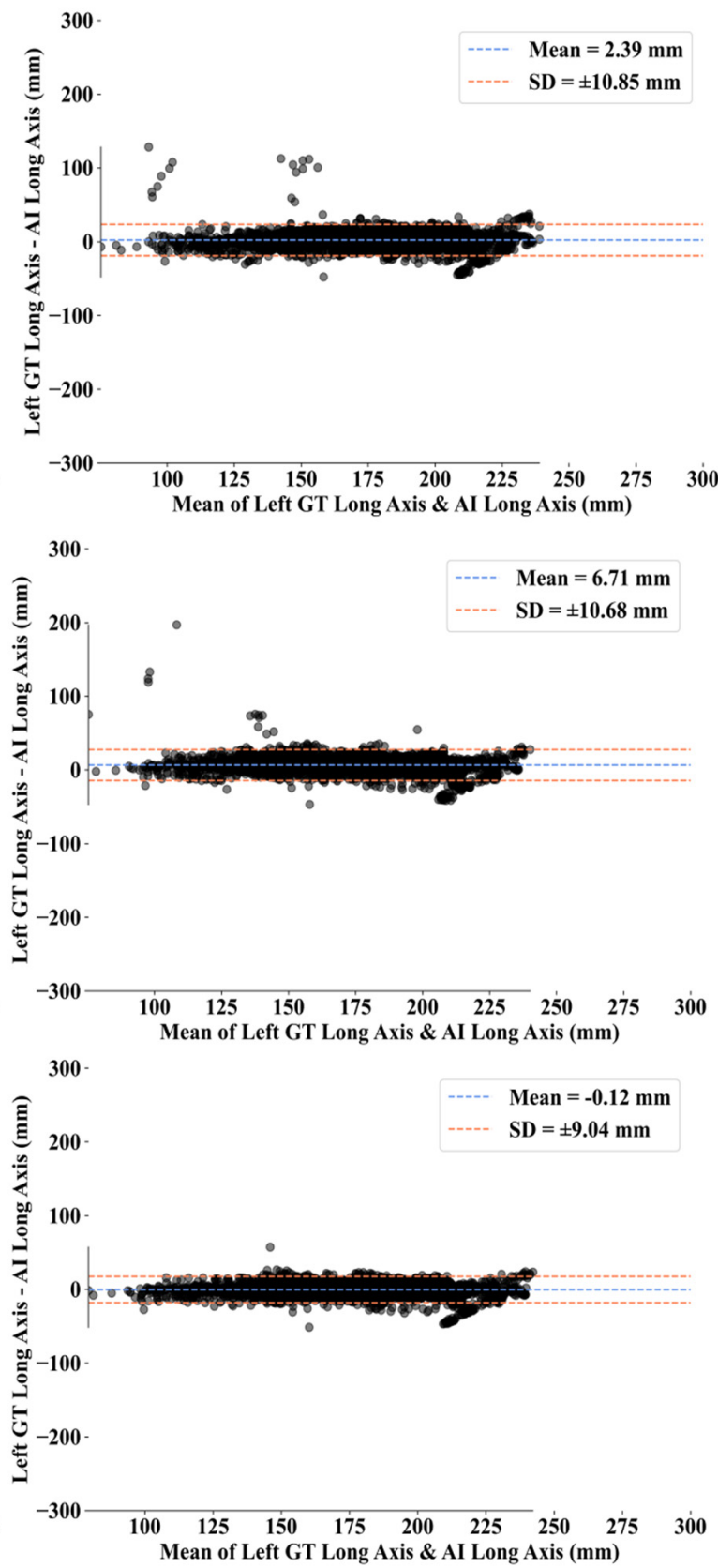

Figure 25. BA for the left LLA using the three: Observer 1 vs. Observer 2. 

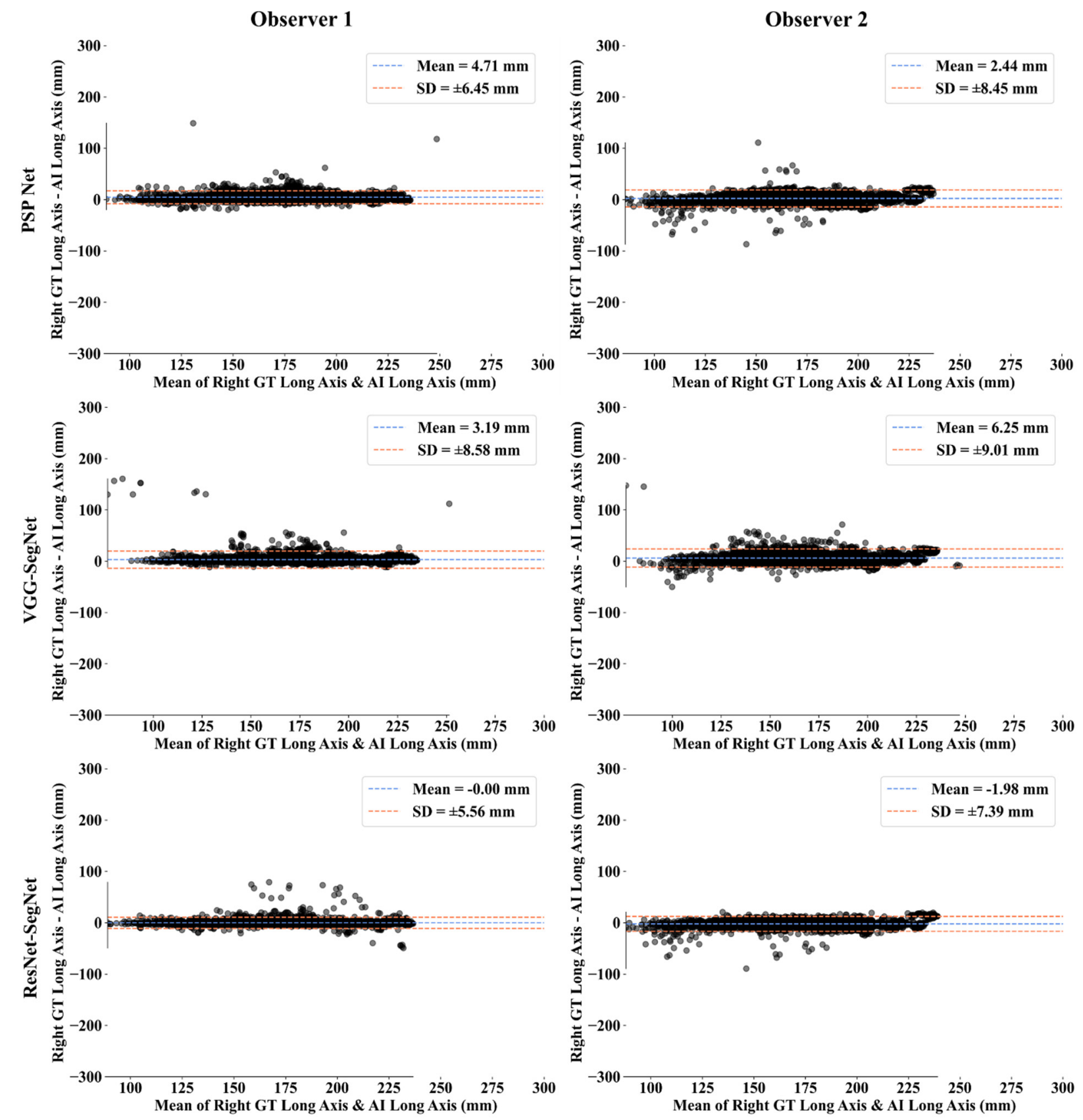

Figure 26. BA for the right LLA using the three AI models: Observer 1 vs. Observer 2.

Statistical Tests

The system's dependability and stability were assessed using a standard paired $t$-test, ANOVA, and Wilcoxon test. The paired $t$-test can be used to see if there is enough data to support a hypothesis; the Wilcoxon test is its alternative when the distribution is not normal. ANOVA helps in the analysis of the difference between the means of groups of the input data. MedCalc software (Osteen, Belgium) was used to perform the statistical analysis. To validate the system presented in this study, we have presented all the possible combinations (twelve in total) for the three AI models between Observer 1 and Observer 2. Table 3 shows the paired $t$-test, ANOVA, and Wilcoxon test results for the 12 combinations. 
Table 3. Paired $t$-test, Wilcoxon, ANOVA, and CC for LA and LLA for the 12 combinations.

\begin{tabular}{|c|c|c|c|c|c|c|c|c|c|}
\hline \multirow[b]{2}{*}{$S N$} & \multirow[b]{2}{*}{ Combinations } & \multicolumn{4}{|c|}{ Lung Area } & \multicolumn{4}{|c|}{ Lung Long Axis } \\
\hline & & $\begin{array}{c}\text { Paired } \\
t \text {-Test } \\
(p \text {-Value })\end{array}$ & $\begin{array}{l}\text { Wilcoxon } \\
\text { ( } p \text {-Value) }\end{array}$ & $\begin{array}{c}\text { ANOVA } \\
(p \text {-Value) }\end{array}$ & $\begin{array}{c}\mathrm{CC} \\
{[0-1]}\end{array}$ & $\begin{array}{c}\text { Paired } \\
t \text {-Test } \\
(p \text {-Value })\end{array}$ & $\begin{array}{l}\text { Wilcoxon } \\
\text { ( } p \text {-Value) }\end{array}$ & $\begin{array}{c}\text { ANOVA } \\
(p \text {-Value) }\end{array}$ & $\begin{array}{c}\text { CC } \\
{[0-1]}\end{array}$ \\
\hline 1 & P1 vs. V1 & $<0.0001$ & $<0.0001$ & $<0.001$ & 0.9726 & $<0.0001$ & $<0.0001$ & $<0.001$ & 0.9509 \\
\hline 2 & P1 vs. R1 & $<0.0001$ & $<0.0001$ & $<0.001$ & 0.9514 & $<0.0001$ & $<0.0001$ & $<0.001$ & 0.9506 \\
\hline 3 & P1 vs. P2 & $<0.0001$ & $<0.0001$ & $<0.001$ & 0.9703 & $<0.0001$ & $<0.0001$ & $<0.001$ & 0.9686 \\
\hline 4 & P1 vs. V2 & $<0.0001$ & $<0.0001$ & $<0.001$ & 0.9446 & $<0.0001$ & $<0.0001$ & $<0.001$ & 0.9445 \\
\hline 5 & P1 vs. R2 & $<0.0001$ & $<0.0001$ & $<0.001$ & 0.9764 & $<0.0001$ & $<0.0001$ & $<0.001$ & 0.9661 \\
\hline 6 & V1 vs. R1 & $<0.0001$ & $<0.0001$ & $<0.001$ & 0.9663 & $<0.0001$ & $<0.0001$ & $<0.001$ & 0.9561 \\
\hline 7 & V1 vs. P2 & $<0.0001$ & $<0.0001$ & $<0.001$ & 0.9726 & $<0.0001$ & $<0.0001$ & $<0.001$ & 0.9671 \\
\hline 8 & V1 vs. V2 & $<0.0001$ & $<0.0001$ & $<0.001$ & 0.9766 & $<0.0001$ & $<0.0001$ & $<0.001$ & 0.9638 \\
\hline 9 & V1 vs. R2 & $<0.0001$ & $<0.0001$ & $<0.001$ & 0.9943 & $<0.0001$ & $<0.0001$ & $<0.001$ & 0.9796 \\
\hline 10 & R1 vs. P2 & $<0.0001$ & $<0.0001$ & $<0.001$ & 0.9549 & $<0.0001$ & $<0.0001$ & $<0.001$ & 0.9617 \\
\hline 11 & R1 vs. V2 & $<0.0001$ & $<0.0001$ & $<0.001$ & 0.9513 & $<0.0001$ & $<0.0001$ & $<0.001$ & 0.9499 \\
\hline 12 & R1 vs. R2 & $<0.0001$ & $<0.0001$ & $<0.001$ & 0.9690 & $<0.0001$ & $<0.0001$ & $<0.001$ & 0.9726 \\
\hline
\end{tabular}

CC: Correlation coefficient; P1: PSP Net for Observer 1; V1: VGG-SegNet for Observer 1; R1: ResNet-SegNet for Observer 1; P2: PSP Net for Observer 2; V2: VGG-SegNet for Observer 2; R2: ResNet-SegNet for Observer 2.

Figure of Merit

The likelihood of the error in the system is known as the figure of merit (FoM). We have calculated FoM for (i) lung area and (ii) lung long axis to show the acceptability of the hypothesis if the \% difference between the two observers is $<5 \%$. Table 4 shows the values for FoM using Equation (5) and the \% difference for the three AI models against the two observers. Similarly, Table 5 shows the values for FoM using Equation (6) and the \% difference for the three AI models against the two observers.

$$
\begin{gathered}
F_{o} M_{A}(m)=100-\left[\left(\frac{\left|\bar{A}_{a i}(m)-\bar{A}_{g t}\right|}{\bar{A}_{g t}}\right) \times 100\right], \\
\operatorname{FoM}_{L A}(m)=100-\left[\left(\frac{\left|\bar{L}_{a i}(m)-\bar{L}_{g t}\right|}{\bar{L}_{g t}}\right) \times 100\right] \\
\text { where } \bar{A}_{a i}(m)=\frac{\sum_{n=1}^{N} A_{a i}(m, n)}{N}, \bar{A}_{g t}=\frac{\sum_{n=1}^{N} A_{g t}(n)}{N}, \\
\overline{L A}_{a i}(m)=\frac{\sum_{n=1}^{N} L A_{a i}(m, n)}{N} \& \overline{L A}_{g t}=\frac{\sum_{n=1}^{N} L A_{g t}(n)}{N}
\end{gathered}
$$

\begin{tabular}{|c|c|c|c|c|c|c|c|c|c|c|c|c|}
\hline & \multicolumn{3}{|c|}{ Observer 1} & \multicolumn{3}{|c|}{ Observer 2} & \multicolumn{3}{|c|}{$\%$ Difference } & \multicolumn{3}{|c|}{ Hypothesis (<5\%) } \\
\hline & Left & Right & Mean & Left & Right & Mean & Left & Right & Mean & Left & Right & Mean \\
\hline PSP Net & 95.07 & 95.11 & 95.09 & 97.37 & 97.49 & 97.43 & $2 \%$ & $3 \%$ & $2 \%$ & $\checkmark$ & $\checkmark$ & $\checkmark$ \\
\hline VGG-SegNet & 96.73 & 97.40 & 97.04 & 97.74 & 97.27 & 97.52 & $1 \%$ & $0 \%$ & $0 \%$ & $\checkmark$ & $\checkmark$ & $\checkmark$ \\
\hline ResNet-SegNet & 98.33 & 99.98 & 99.11 & 97.88 & 99.20 & 98.50 & $0 \%$ & $1 \%$ & $1 \%$ & $\checkmark$ & $\checkmark$ & $\checkmark$ \\
\hline
\end{tabular}

Table 4. FoM for lung area. 
Table 5. FoM for lung long axis.

\begin{tabular}{|c|c|c|c|c|c|c|c|c|c|c|c|c|}
\hline & \multicolumn{3}{|c|}{ Observer 1} & \multicolumn{3}{|c|}{ Observer 2} & \multicolumn{3}{|c|}{ \% Difference } & \multicolumn{3}{|c|}{ Hypothesis $(<5 \%)$} \\
\hline & Left & Right & Mean & Left & Right & Mean & Left & Right & Mean & Left & Right & Mean \\
\hline PSP Net & 98.91 & 97.34 & 98.13 & 98.65 & 98.60 & 98.62 & $0 \%$ & $1 \%$ & $1 \%$ & $\checkmark$ & $\checkmark$ & $\checkmark$ \\
\hline VGG-SegNet & 99.41 & 98.50 & 98.95 & 97.07 & 97.27 & 97.17 & $2 \%$ & $1 \%$ & $2 \%$ & $\checkmark$ & $\checkmark$ & $\checkmark$ \\
\hline ResNet-SegNet & 99.73 & 99.37 & 99.83 & 99.51 & 98.75 & 99.13 & $0 \%$ & $1 \%$ & $1 \%$ & $\checkmark$ & $\checkmark$ & $\checkmark$ \\
\hline
\end{tabular}

\section{Discussion}

The study presented the inter-observer variability analysis for the COVLIAS 1.0 using three AI models, PSP Net, VGG-SegNet, and ResNet-SegNet. These models have considered tissue characterization approaches since they analyze the tissue data for better feature extraction to evaluate for ground vs. background, thus are more akin to a tissue characterization in classification framework $[30,37]$. Our group has strong experience in tissue characterization approaches with different AI models and applications for classification using ML frameworks such as plaque, liver, thyroid, breast [21,28,30,63-68], and DL framework $[1,36,69,70]$. These three AI models were trained using the GT annotated data from the two observers. The percentage difference between the outputs of the two AI model results was less than $5 \%$, and thus the hypothesis was confirmed. During the training, the K5 cross-validation protocol was adapted on a set of $5000 \mathrm{CT}$ images. For the PE of the proposed inter-observer variability system, the following ten metrics were considered: (i) visualization of the lung boundary, (ii) visualization of the lung long axis, cumulative frequency plots for (iii) LAE, (iv) LLAE, CC plots for (v) lung area, (vi) lung long axis, BA plots for (vii) lung area, (viii) lung long axis, (ix) ROC and AUC curve, and (x) JI and DS for estimated AI model lung regions. These matrices showed consistent and stable results. The training, evaluation, and quantification were implemented on the GPU environment (DGX V100) using python. We adapted vectorization provided by python during the implementation of the Numba library.

\subsection{A Special Note on Three Model Behaviors with Respect to the Two OBSERVERS}

The proposed inter-observer variability study used three AI models for the analysis, where PSP Net was implemented for the first time for COVID-19 lung segmentation. The other models VGG-SegNet and ResNet-SegNet were used for benchmarking. The AUC for the mean lung region for the three AI models was $>0.95$ for both Observer 1 and Observer 2 .

Our results, shown below in Table 6, compared various metrics that included the interobserver variability study for the three AI models. All the models behaved consistently while using the two different observers. Our results showed that ResNet-SegNet was the best performing model for all the PE metrics. The percentage difference between the two observers was $0.4 \%, 3.7 \%$, and $0.4 \%$, respectively, for the three models PSP Net, VGGSegNet, and ResNet-SegNet, respectively. This further validated our hypothesis for every AI model, keeping the error threshold less than 5\%. Even though all three AI models passed the hypothesis, VGG-SegNet is the least superior. This is because the number of the layers in the VGG-SegNet architecture (Figure 5) is 19, compared to 50 in PSP Net (Figure 4) and 51 (encoder part) in the ResNet-SegNet model (Figure 6). By taking the results from both the observers into account, the order of the performance of the models is ResNet-SegNet $>$ PSP Net $>$ VGG-SegNet. Further, we also conclude that HDL models are superior to SDL (PSP Net). The aggregate score was computed as the mean for all the models for Observer 1, Observer 2, and the mean of the two Observers. Even though the performance of all the models was comparable, when carefully looking at the performance of Observer 1 the order of performance was ResNet-SegNet $>$ VGG-SegNet $>$ PSP Net. For Observer 2, the order of performance was ResNet-SegNet $>$ PSP Net $>$ VGG-SegNet. Further, the performance of the left lung was better than the right lung for the reasons unclear at this point, and more investigations would be needed to evaluate this. 
Table 6. Comparison of PE metrics for Observer 1 and Observer 2 and their mean.

\begin{tabular}{ccccccccccc}
\hline & \multicolumn{3}{c}{ Observer 1 } & \multicolumn{3}{c}{ Observer 2 } & \multicolumn{3}{c}{ Mean Obs. 1 \& Obs. 2 } \\
\hline \multirow{2}{*}{ Attributes } & $\begin{array}{c}\text { PSP } \\
\text { Net }\end{array}$ & $\begin{array}{c}\text { VGG- } \\
\text { SegNet }\end{array}$ & $\begin{array}{c}\text { ResNet- } \\
\text { SegNet }\end{array}$ & $\begin{array}{c}\text { PSP } \\
\text { Net }\end{array}$ & $\begin{array}{c}\text { VGG- } \\
\text { SegNet }\end{array}$ & $\begin{array}{c}\text { ResNet- } \\
\text { SegNet }\end{array}$ & $\begin{array}{c}\text { PSP } \\
\text { Net }\end{array}$ & $\begin{array}{c}\text { VGG- } \\
\text { SegNet }\end{array}$ & $\begin{array}{c}\text { ResNet- } \\
\text { SegNet }\end{array}$ \\
\hline DS & 0.96 & 0.98 & 0.98 & 0.96 & 0.95 & 0.97 & 0.96 & 0.97 & 0.98 \\
\hline JI & 0.93 & 0.96 & 0.97 & 0.92 & 0.9 & 0.94 & 0.93 & 0.93 & 0.96 \\
\hline CC Left LA & 0.98 & 0.98 & 0.98 & 0.98 & 0.98 & 1 & 0.98 & 0.98 & 0.99 \\
\hline CC Right LA & 0.98 & 0.99 & 0.98 & 0.98 & 0.98 & 1 & 0.98 & 0.99 & 0.99 \\
\hline CC Left LLA & 0.97 & 0.96 & 0.98 & 0.96 & 0.96 & 0.98 & 0.97 & 0.96 & 0.98 \\
\hline CC Right LLA & 0.99 & 0.97 & 0.99 & 0.98 & 0.97 & 0.98 & 0.99 & 0.97 & 0.99 \\
\hline CF Left LA < 10\% & 0.83 & 0.85 & 0.90 & 0.81 & 0.75 & 0.89 & 0.82 & 0.80 & 0.89 \\
\hline CF Right LA < 10\% & 0.78 & 0.85 & 0.90 & 0.80 & 0.75 & 0.88 & 0.79 & 0.80 & 0.89 \\
\hline Aggregate Score & $\mathbf{7 . 4 2}$ & $\mathbf{7 . 5 4}$ & $\mathbf{7 . 6 7}$ & $\mathbf{7 . 3 9}$ & $\mathbf{7 . 2 4}$ & $\mathbf{7 . 6 4}$ & $\mathbf{7 . 4 0}$ & $\mathbf{7 . 3 9}$ & $\mathbf{7 . 6 6}$ \\
\hline
\end{tabular}

DS: Dice similarity; JI: Jaccard index; CC: Correlation coefficient; LA: Lung area; LLA: Lung long axis; CF: Cumulative frequency; Obs: Observer.

\subsection{Benchmarking}

There have been several studies in the area of DL for lung segmentation, but only a few in the region of COVID-19 [71-74], and even less that involved variability analysis. Table 7 shows the benchmarking table having three variability studies: Saba et al. [48], Jeremy et al. [75], and Joskowicz et al. [76], that are compared against Suri et al. in this proposed study. Saba et al. has used a dataset of 96 patients with three observers for tracings, and ROC curves were also not presented in the study. Jeremy et al. [60] have demonstrated the variability analysis using five different observers that used the area error as the metric. The boundary error, ROC, JI, and DS were not discussed. Finally, Joskowicz et al. [76] used 480 images and 11 observers to annotate the dataset, but no area and boundary errors were present. Moreover, they did not present the ROC curves, JI, and DS for the tracings. All three studies $[48,75,76]$, only performed manual annotation of the non-COVID dataset, and there was no involvement of the AI techniques to generate the boundaries automatically. Comparatively, the proposed study provides a first-of-its-kind for inter-observer variability analysis alongside HDL and SDL solutions, supporting our hypothesis that the error between the AI models trained using the two observers involved is less than $5 \%$.

\subsection{Strengths, Weakness, and Extensions}

The proposed study successfully validated the hypothesis for the inter-observer variability settings, demonstrating that the difference between the two AI models when trained by the two observers was less than $5 \%$. It was the first-time inter-observer variability was presented for COVID-19 lung segmentation using HDL and SDL models.

In spite of encouraging results, the study could not include more than two observers due to reasons such as cost, time, and availability of the radiologists. The imaging analysis component could be extended to handle more dense pulmonary opacities such as consolidation or mixed opacities during lung segmentation.

As part of the extension, the HDL models can be extended, which combines DL with ML or two solo DL models for lung segmentation. Conventional methods [77,78] can be used for lung segmentation embedded with denoising methods [79] and benchmarked against the AI models. The system can be extended to unseen data where the training data is taken from one clinical site and testing data can be from the other clinical site. It would also be interesting to explore the segmentation of lungs in the healthy patients using the AI model trained on COVID-19 patients. Other neural network techniques such as generative adversarial networks (GANs) [80] or transfer learning and loss schemes [38,44,81] can also 
be adapted. A big data framework can be used to integrate comorbidity factors [82] in the AI models.

Table 7. Benchmarking Table.

\begin{tabular}{|c|c|c|c|c|}
\hline Attributes/Author & Saba et al. [49] & Jeremy et al. [77] & Joskowicz et al. [78] & Suri et al. (Proposed) \\
\hline \# of patients & 96 & 33 & 18 & 72 \\
\hline \# of Images & NA & NA & 490 & 5000 \\
\hline \# of Observers & 3 & 5 & 11 & 2 \\
\hline Dataset & Non-COVID & Non-COVID & Non-COVID & COVID \\
\hline Image Size & 512 & NA & 512 & 768 \\
\hline$\#$ of tests/PE & 5 & 0 & 2 & 13 \\
\hline $\mathrm{CC}$ & 0.98 & NA & NA & 0.98 \\
\hline Boundary estimation & Manual & Manual & Manual & Manual \& automatic \\
\hline AI Models & NA & NA & NA & 3 \\
\hline Modality & $\mathrm{CT}$ & $\mathrm{CT}$ & $\mathrm{CT}$ & $\mathrm{CT}$ \\
\hline Area Error & $\checkmark$ & $\checkmark$ & $x$ & $\checkmark$ \\
\hline Boundary Error & $\checkmark$ & $x$ & $x$ & $\checkmark$ \\
\hline ROC & $x$ & $x$ & $x$ & $\checkmark$ \\
\hline JI & $\checkmark$ & $x$ & $x$ & $\checkmark$ \\
\hline DS & $\checkmark$ & $x$ & $x$ & $\checkmark$ \\
\hline
\end{tabular}

CC: Correlation coefficient; ROC: Receiver-Operating Characteristics; DS: Dice similarity; JI: Jaccard index.

\section{Conclusions}

The proposed study is the first of its kind to evaluate the effect of ground-truth tracings on the AI models for COVID-19 CT lung segmentation. Three kinds of AI models, PSP Net, VGG-SegNet, and ResNet-SegNet, were adapted for lung segmentation. Two different Observers were used to annotate 5000 CT lung slices taken from 72 COVID-19 patients. Thus, six AI training models (three AI models times two Observers) were generated and evaluated using the K5 cross-validation protocol. Ten different kinds of metrics were used for the evaluation of the six AI models. The two Observers' error metrics were compared to validate the hypothesis for every AI model, keeping below the error threshold of $5 \%$. Our results showed that the difference in these errors were $0 \%, 0.51 \%$, and $2.04 \%($ all $<5 \%$, respectively, for the three AI models, validating the hypothesis. Statistical analysis was conducted using a standard paired t-test, ANOVA, and Wilcoxon test to prove the system's hypothesis. The inter-variability COVLIAS 1.0 showed clinically robust and statistically stable outcomes for this pilot study and, thus, can be adapted in clinical settings.

Author Contributions: Conceptualization: J.S.S., M.K.K., N.N.K. and M.M.; Data Curation: M.C., L.S., G.F., M.T., K.V., P.R.K., F.N., Z.R. and A.G.; Formal Analysis: J.S.S., M.K.K.; Investigation: J.S.S., M.K.K., I.M.S., P.S.C., A.M.J., S.M., J.R.L., G.P., D.W.S., P.P.S., G.T., A.P., D.P.M., V.A., J.S.T., M.A.-M., S.K.D., A.N., A.S. and A.A.; Methodology: J.S.S., S.A. and A.B.; Project Administration: J.S.S., M.K.K.; Deep Learning Computing Resources: S.K.G.; Software Design and Usage: S.A., P.E. and V.K.; Software Verification: J.S.S., L.S. and M.K.K.; Supervision: J.S.S., M.K.K. and S.N.; Scientific Validation: J.S.S. and S.A.; Clinical Validation and Discussions: J.S.S., M.K.K. and L.S.; Visualization: S.A.; Writing-Original Draft: J.S.S. and S.A.; Writing-Review \& Editing: J.S.S., M.K.K., L.S., R.P., S.K.G., I.M.S., M.T., P.S.C., A.M.J., N.N.K., K.V., S.M., J.R.L., G.P., M.M., D.W.S., A.B., P.P.S., G.T., A.P., D.P.M., V.A., G.D.K., M.A.-M., S.K.D., A.N., A.S., V.R., M.F., F.N., A.G. and S.N. All authors have read and agreed to the published version of the manuscript.

Funding: This research received no external funding.

Institutional Review Board Statement: Not applicable. 
Informed Consent Statement: Not applicable.

Data Availability Statement: Not applicable.

Conflicts of Interest: The authors declare no conflict of interest. GBTI deals in lung image analysis and Jasjit S. Suri is affiliated with GBTI.

\section{Abbreviations}

\section{SN Symbol Description of the Symbols}

$$
\text { ACC (ai) Accuracy }
$$

AE Area Error

AI Artificial Intelligence

ARDS Acute Respiratory Distress Syndrome

AUC Area Under the Curve

BA Bland-Altman

BE Boundary Error

CC Correlation coefficient

CE Cross Entropy

COVID Coronavirus disease

COVLIAS COVID Lung Image Analysis System

CT Computed Tomography

DL Deep Learning

DS Dice Similarity

FoM Figure of merit

GT Ground Truth

HDL Hybrid Deep Learning

IS Image Size

JI Jaccard Index

LAE Lung Area Error

LLAE Lung Long Axis Error

$\mathrm{NIH} \quad$ National Institute of Health

PC Pixel Counting

RF Resolution Factor

ROC Receiver operating characteristic

SDL Solo Deep Learning

VGG Visual Geometric Group

VS Variability studies

WHO World Health Organization

\section{Symbols}

$\begin{array}{ll}\text { SN } & \text { Symbol } \\ 1 & l_{C E} \\ 2 & \mathrm{~m} \\ 3 & \mathrm{n} \\ 4 & \bar{A}_{a i}(m) \\ 5 & A_{a i}(m, n) \\ 6 & A_{g t}(n) \\ 7 & \bar{A}_{g t} \\ 8 & \overline{L A}_{a i}(m) \\ 9 & L A_{a i}(m, n) \\ 10 & \operatorname{LA}_{g t}(n) \\ 11 & \overline{L A}_{g t} \\ 12 & \text { FoM } \\ A & (m) \\ 14 & \text { FoM } \\ L A & (m) \\ 15 & \mathrm{JI} \\ 16 & \text { DSC }\end{array}$

\section{Description of the Symbols}

Cross Entropy-loss

Model used for segmentation in the total number of models $\mathrm{M}$

Image scan number in total number $N$

Mean estimated lung area for all images using AI model ' $m$ '

Estimated Lung Area using AI model ' $\mathrm{m}$ ' and image ' $n$ '

GT lung area for image ' $n$ '

Mean ground truth area for all images $\mathrm{N}$ in the database

Mean estimated lung long axis for all images using AI model ' $m$ '

Estimated lung long axis using AI model ' $m$ ' and image ' $n$ '

GT lung long axis for image ' $n$ '

Mean ground truth long axis for all images $\mathrm{N}$ in the database

Figure-of-Merit for segmentation model ' $m$ '

Figure-of-Merit for long axis for model ' $m$ '

Jaccard Index for a specific segmentation model

Dice Similarity Coefficient for a specific segmentation model 


$\begin{array}{lll}17 & \text { TP, TN } & \text { True Positive and True Negative } \\ 18 & \text { FP, FN } & \text { False Positive and False Negative } \\ 19 & x_{\mathrm{i}} & \text { GT label } \\ 20 & \mathrm{p}_{\mathrm{i}} & \text { SoftMax classifier probability } \\ 21 & Y_{p} & \text { Ground truth image } \\ 22 & \hat{Y}_{p} & \text { Estimated image } \\ 23 & P & \text { Total no of pixels in an image in } x, y \text {-direction } \\ 24 & \text { K5 } & \text { Cross-validation protocol with } 80 \% \text { training and } 20 \% \text { testing (5 folds) } \\ \text { Deep } & \text { Learning Segmentation Architectures } \\ 25 & \text { PSP Net } & \text { SDL model for lung segmentation with pyramidal feature extraction } \\ 26 & \text { VGG-SegNet } & \text { HDL model designed by fusion of VGG-19 and SegNet architecture } \\ 27 & \text { ResNet-SegNet } & \text { HDL model designed by fusion of ResNet-50 and SegNet architecture }\end{array}$

\section{References}

1. Agarwal, M.; Saba, L.; Gupta, S.K.; Johri, A.M.; Khanna, N.N.; Mavrogeni, S.; Laird, J.R.; Pareek, G.; Miner, M.; Sfikakis, P.P. Wilson disease tissue classification and characterization using seven artificial intelligence models embedded with 3D optimization paradigm on a weak training brain magnetic resonance imaging datasets: A supercomputer application. Med. Biol. Eng. Comput. 2021, 59, 511-533. [CrossRef]

2. Cau, R.; Pacielli, A.; Fatemeh, H.; Vaudano, P.; Arru, C.; Crivelli, P.; Stranieri, G.; Suri, J.S.; Mannelli, L.; Conti, M.; et al. Complications in COVID-19 patients: Characteristics of pulmonary embolism. Clin. Imaging 2021, 77, 244-249. [CrossRef] [PubMed]

3. Saba, L.; Gerosa, C.; Fanni, D.; Marongiu, F.; La Nasa, G.; Caocci, G.; Barcellona, D.; Balestrieri, A.; Coghe, F.; Orru, G.; et al. Molecular pathways triggered by COVID-19 in different organs: ACE2 receptor-expressing cells under attack? A review. Eur. Rev. Med. Pharmacol. Sci. 2020, 24, 12609-12622.

4. Cau, R.; Bassareo, P.P.; Mannelli, L.; Suri, J.S.; Saba, L. Imaging in COVID-19-related myocardial injury. Int. J. Cardiovasc. Imaging 2021, 37, 1349-1360. [CrossRef] [PubMed]

5. Viswanathan, V.; Viswanathan, V.; Puvvula, A.; Jamthikar, A.D.; Saba, L.; Johri, A.M.; Kotsis, V.; Khanna, N.N.; Dhanjil, S.K.; Majhail, M.; et al. Bidirectional link between diabetes mellitus and coronavirus disease 2019 leading to cardiovascular disease: A narrative review. World J. Diabetes 2021, 12, 215-237. [CrossRef]

6. Suri, J.S.; Agarwal, S.; Gupta, S.K.; Puvvula, A.; Biswas, M.; Saba, L.; Bit, A.; Tandel, G.S.; Agarwal, M.; Patrick, A.; et al. A narrative review on characterization of acute respiratory distress syndrome in COVID-19-infected lungs using artificial intelligence. Comput. Biol. Med. 2021, 130, 104210. [CrossRef]

7. Cau, R.; Falaschi, Z.; Paschè, A.; Danna, P.; Arioli, R.; Arru, C.D.; Zagaria, D.; Tricca, S.; Suri, J.S.; Karla, M.K.; et al. Computed tomography findings of COVID-19 pneumonia in Intensive Care Unit-patients. J. Public Health Res. 2021, 10, 2270. [CrossRef]

8. $\quad$ Emery, S.L.; Erdman, D.D.; Bowen, M.D.; Newton, B.R.; Winchell, J.M.; Meyer, R.F.; Tong, S.; Cook, B.T.; Holloway, B.P.; McCaustland, K.A.; et al. Real-time reverse transcription-polymerase chain reaction assay for SARS-associated coronavirus. Emerg. Infect. Dis. 2004, 10, 311-316. [CrossRef] [PubMed]

9. Wu, X.; Hui, H.; Niu, M.; Li, L.; Wang, L.; He, B.; Yang, X.; Li, L.; Li, H.; Tian, J.; et al. Deep learning-based multi-view fusion model for screening 2019 novel coronavirus pneumonia: A multicentre study. Eur. J. Radiol. 2020, 128, 109041. [CrossRef] [PubMed]

10. Pathak, Y.; Shukla, P.K.; Tiwari, A.; Stalin, S.; Singh, S. Deep transfer learning based classification model for COVID-19 disease. IRBM 2020, in press. [CrossRef]

11. Saba, L.; Suri, J.S. Multi-Detector CT Imaging: Principles, Head, Neck, and Vascular Systems; CRC Press: Boca Raton, FL, USA, 2013; Volume 1.

12. Gozes, O.; Frid-Adar, M.; Greenspan, H.; Browning, P.D.; Zhang, H.; Ji, W.; Bernheim, A.; Siegel, E. Rapid ai development cycle for the coronavirus (COVID-19) pandemic: Initial results for automated detection \& patient monitoring using deep learning ct image analysis. arXiv 2020, arXiv:05037.

13. Shalbaf, A.; Vafaeezadeh, M. Automated detection of COVID-19 using ensemble of transfer learning with deep convolutional neural network based on CT scans. Int. J. Comput. Assist. Radiol. Surg. 2021, 16, 115-123.

14. Yang, X.; He, X.; Zhao, J.; Zhang, Y.; Zhang, S.; Xie, P. COVID-CT-dataset: A CT scan dataset about COVID-19. arXiv 2020, arXiv:13865.

15. Alqudah, A.M.; Qazan, S.; Alquran, H.; Qasmieh, I.A.; Alqudah, A. COVID-2019 Detection Using X-ray Images and Artificial Intelligence Hybrid Systems. Phys. Sci. 2020, 2, 1.

16. Aslan, M.F.; Unlersen, M.F.; Sabanci, K.; Durdu, A. CNN-based transfer learning-BiLSTM network: A novel approach for COVID-19 infection detection. Appl. Soft Comput. 2021, 98, 10691. [CrossRef]

17. Wu, Y.H.; Gao, S.H.; Mei, J.; Xu, J.; Fan, D.P.; Zhang, R.G.; Cheng, M.M. Jcs: An explainable COVID-19 diagnosis system by joint classification and segmentation. IEEE Trans. Image Process. 2021, 30, 3113-3126. [CrossRef] [PubMed]

18. Xu, X.; Jiang, X.; Ma, C.; Du, P.; Li, X.; Lv, S.; Yu, L.; Ni, Q.; Chen, Y.; Su, J.; et al. A deep learning system to screen novel coronavirus disease 2019 pneumonia. Engineering 2020, 6, 1122-1129. [CrossRef] [PubMed] 
19. El-Baz, A.; Suri, J. Machine Learning in Medicine; CRC Press: Boca Raton, FL, USA, 2021; ISBN 9781138106901.

20. Suri, J.S.; Rangayyan, R.M. Recent Advances in Breast Imaging, Mammography, and Computer-Aided Diagnosis of Breast Cancer; SPIE Publications: Bellingham, WA, USA, 2006.

21. Biswas, M.; Kuppili, V.; Edla, D.R.; Suri, H.S.; Saba, L.; Marinhoe, R.T.; Sanches, J.M.; Suri, J.S. Symtosis: A liver ultrasound tissue characterization and risk stratification in optimized deep learning paradigm. Comput. Methods Programs Biomed. 2018, 155, 165-177. [CrossRef] [PubMed]

22. Acharya, U.R.; Sree, S.V.; Ribeiro, R.; Krishnamurthi, G.; Marinho, R.T.; Sanches, J.; Suri, J.S. Data mining framework for fatty liver disease classification in ultrasound: A hybrid feature extraction paradigm. Med. Phys. 2012, 39, 4255-4264. [CrossRef] [PubMed]

23. Acharya, U.R.; Sree, S.V.; Krishnan, M.M.R.; Molinari, F.; Garberoglio, R.; Suri, J.S. Non-invasive automated 3D thyroid lesion classification in ultrasound: A class of ThyroScan ${ }^{\mathrm{TM}}$ systems. Ultrasonics 2012, 52, 508-520. [CrossRef]

24. Acharya, U.R.; Swapna, G.; Sree, S.V.; Molinari, F.; Gupta, S.; Bardales, R.H.; Witkowska, A.; Suri, J.S. A review on ultrasoundbased thyroid cancer tissue characterization and automated classification. Technol. Cancer Res. Treat. 2014, 13, 289-301. [CrossRef]

25. Molinari, F.; Mantovani, A.; Deandrea, M.; Limone, P.; Garberoglio, R.; Suri, J.S. Characterization of single thyroid nodules by contrast-enhanced 3-D ultrasound. Ultrasound Med. Biol. 2010, 36, 1616-1625. [CrossRef]

26. Shrivastava, V.K.; Londhe, N.D.; Sonawane, R.S.; Suri, J.S. Computer-aided diagnosis of psoriasis skin images with HOS, texture and color features: A first comparative study of its kind. Comput. Methods Programs Biomed. 2016, 126, 98-109. [CrossRef] [PubMed]

27. Shrivastava, V.K.; Londhe, N.D.; Sonawane, R.S.; Suri, J.S. Reliable and accurate psoriasis disease classification in dermatology images using comprehensive feature space in machine learning paradigm. Expert Syst. Appl. 2015, 42, 6184-6195. [CrossRef]

28. Pareek, G.; Acharya, U.R.; Sree, S.V.; Swapna, G.; Yantri, R.; Martis, R.J.; Saba, L.; Krishnamurthi, G.; Mallarini, G.; El-Baz, A.; et al. Prostate tissue characterization/classification in 144 patient population using wavelet and higher order spectra features from transrectal ultrasound images. Technol. Cancer Res. Treat. 2013, 12, 545-557. [CrossRef]

29. McClure, P.; Elnakib, A.; El-Ghar, M.A.; Khalifa, F.; Soliman, A.; El-Diasty, T.; Suri, J.S.; Elmaghraby, A.; El-Baz, A. In-vitro and in-vivo diagnostic techniques for prostate cancer: A review. J. Biomed. Nanotechnol. 2014, 10, 2747-2777. [CrossRef] [PubMed]

30. Mookiah, M.R.K.; Acharya, U.R.; Martis, R.J.; Chua, C.K.; Lim, C.M.; Ng, E.Y.K.; Laude, A. Evolutionary algorithm based classifier parameter tuning for automatic diabetic retinopathy grading: A hybrid feature extraction approach. Knowl.-Based Syst. 2013, 39, 9-22. [CrossRef]

31. Than, J.C.; Saba, L.; Noor, N.M.; Rijal, O.M.; Kassim, R.M.; Yunus, A.; Suri, H.S.; Porcu, M.; Suri, J.S. Lung disease stratification using amalgamation of Riesz and Gabor transforms in machine learning framework. Comput. Biol. Med. 2017, 89, 197-211. [CrossRef] [PubMed]

32. El-Baz, A.; Jiang, X.; Suri, J.S. Biomedical Image Segmentation: Advances and Trends; CRC Press: Boca Raton, FL, USA, 2016.

33. Than, J.C.; Saba, L.; Noor, N.M.; Rijal, O.M.; Kassim, R.M.; Yunus, A.; Suri, H.S.; Porcu, M.; Suri, J.S. Shape recovery algorithms using level sets in 2-D/3-D medical imagery: A state-of-the-art review. IEEE Trans. Inf. Technol. Biomed. 2002, 6, 8-28.

34. El-Baz, A.S.; Acharya, R.; Mirmehdi, M.; Suri, J.S. Multi Modality State-of-the-Art Medical Image Segmentation and Registration Methodologies; Springer Science \& Business Media: Berlin, Germany, 2011; Volume 2.

35. El-Baz, A.; Suri, J.S. Level Set Method in Medical Imaging Segmentation; CRC Press: Boca Raton, FL, USA, 2019.

36. Saba, L.; Sanagala, S.S.; Gupta, S.K.; Koppula, V.K.; Johri, A.M.; Khanna, N.N.; Mavrogeni, S.; Laird, J.R.; Pareek, G.; Miner, M.; et al. Multimodality carotid plaque tissue characterization and classification in the artificial intelligence paradigm: A narrative review for stroke application. Ann. Transl. Med. 2021, 9, 1206. [CrossRef] [PubMed]

37. Acharya, U.R.; Sree, S.V.; Krishnan, M.M.R.; Krishnananda, N.; Ranjan, S.; Umesh, P.; Suri, J.S. Automated classification of patients with coronary artery disease using grayscale features from left ventricle echocardiographic images. Comput. Methods Programs Biomed. 2013, 112, 624-632. [CrossRef]

38. Agarwal, M.; Saba, L.; Gupta, S.K.; Carriero, A.; Falaschi, Z.; Paschè, A.; Danna, P.; El-Baz, A.; Naidu, S.; Suri, J.S. A novel block imaging technique using nine artificial intelligence models for COVID-19 disease classification, characterization and severity measurement in lung computed tomography scans on an Italian cohort. J. Med. Syst. 2021, 45, 1-30. [CrossRef]

39. Saba, L.; Agarwal, M.; Patrick, A.; Puvvula, A.; Gupta, S.K.; Carriero, A.; Laird, J.R.; Kitas, G.D.; Johri, A.M.; Balestrieri, A.; et al. Six artificial intelligence paradigms for tissue characterisation and classification of non-COVID-19 pneumonia against COVID-19 pneumonia in computed tomography lungs. Int. J. Comput. Assist. Radiol. Surg. 2021, 16, 423-434. [CrossRef]

40. Skandha, S.S.; Gupta, S.K.; Saba, L.; Koppula, V.K.; Johri, A.M.; Khanna, N.N.; Mavrogeni, S.; Laird, J.R.; Pareek, G.; Miner, M.; et al. 3-D optimized classification and characterization artificial intelligence paradigm for cardiovascular/stroke risk stratification using carotid ultrasound-based delineated plaque: Atheromatic ${ }^{\mathrm{TM}}$ 2.0. Comput. Biol. Med. 2020, 125, 103958. [CrossRef]

41. Tandel, G.S.; Balestrieri, A.; Jujaray, T.; Khanna, N.N.; Saba, L.; Suri, J.S. Multiclass magnetic resonance imaging brain tumor classification using artificial intelligence paradigm. Comput. Biol. Med. 2020, 122, 103804. [CrossRef] [PubMed]

42. Sarker, M.M.K.; Makhlouf, Y.; Banu, S.F.; Chambon, S.; Radeva, P.; Puig, D. Web-based efficient dual attention networks to detect COVID-19 from X-ray images. Electron. Lett. 2020, 56, 1298-1301. [CrossRef]

43. Sarker, M.M.K.; Makhlouf, Y.; Craig, S.G.; Humphries, M.P.; Loughrey, M.; James, J.A.; Salto-Tellez, M.; O’Reilly, P.; Maxwell, P. A Means of Assessing Deep Learning-Based Detection of ICOS Protein Expression in Colon Cancer. Cancers 2021, $13,3825$. [CrossRef] [PubMed] 
44. Jain, P.K.; Sharma, N.; Giannopoulos, A.A.; Saba, L.; Nicolaides, A.; Suri, J.S. Hybrid deep learning segmentation models for atherosclerotic plaque in internal carotid artery B-mode ultrasound. Comput. Biol. Med. 2021, 136, 104721. [CrossRef]

45. Jena, B.; Saxena, S.; Nayak, G.K.; Saba, L.; Sharma, N.; Suri, J.S. Artificial Intelligence-based Hybrid Deep Learning Models for Image Classification: The First Narrative Review. Comput. Biol. Med. 2021, 137, 104803. [CrossRef] [PubMed]

46. Suri, J.; Agarwal, S.; Gupta, S.K.; Puvvula, A.; Viskovic, K.; Suri, N.; Alizad, A.; El-Baz, A.; Saba, L.; Fatemi, M.; et al. Systematic Review of Artificial Intelligence in Acute Respiratory Distress Syndrome for COVID-19 Lung Patients: A Biomedical Imaging Perspective. IEEE J. Biomed. Health Inform. 2021, 25.

47. Saba, L.; Banchhor, S.K.; Araki, T.; Viskovic, K.; Londhe, N.D.; Laird, J.R.; Suri, H.S.; Suri, J.S. Intra- and inter-operator reproducibility of automated cloud-based carotid lumen diameter ultrasound measurement. Indian Heart J. 2018, 70, 649-664. [CrossRef] [PubMed]

48. Saba, L.; Than, J.C.; Noor, N.M.; Rijal, O.M.; Kassim, R.M.; Yunus, A.; Ng, C.R.; Suri, J.S. Inter-observer Variability Analysis of Automatic Lung Delineation in Normal and Disease Patients. J. Med. Syst. 2016, 40, 142. [CrossRef]

49. Zhang, S.; Suri, J.S.; Salvado, O.; Chen, Y.; Wacker, F.K.; Wilson, D.L.; Duerk, J.L.; Lewin, J.S. Inter-and Intra-Observer Variability Assessment of in Vivo Carotid Plaque Burden Quantification Using Multi-Contrast Dark Blood MR Images. Stud. Health Technol. Inform. 2005, 113, 384-393. [PubMed]

50. Aggarwal, D.; Saini, V. Factors limiting the utility of bronchoalveolar lavage in the diagnosis of COVID-19. Eur. Respir. J. 2020, 56, 2003116. [CrossRef]

51. Saba, L.; Banchhor, S.K.; Suri, H.S.; Londhe, N.D.; Araki, T.; Ikeda, N.; Viskovic, K.; Shafique, S.; Laird, J.R.; Gupta, A.; et al. Accurate cloud-based smart IMT measurement, its validation and stroke risk stratification in carotid ultrasound: A web-based point-of-care tool for multicenter clinical trial. Comput. Biol. Med. 2016, 75, 217-234. [CrossRef]

52. Zhao, H.; Shi, J.; Qi, X.; Wang, X.; Jia, J. Pyramid scene parsing network. In Proceedings of the IEEE Conference on Computer Vision and Pattern Recognition, Honolulu, HI, USA, 21-26 July 2017; pp. 2881-2890.

53. Simonyan, K.; Zisserman, A. Very deep convolutional networks for large-scale image recognition. arXiv 2014, arXiv:1409.1556.

54. Suri, J.S.; Agarwal, S.; Pathak, R.; Ketireddy, V.; Columbu, M.; Saba, L.; Gupta, S.K.; Faa, G.; Singh, I.M.; Turk, M.; et al. COVLIAS 1.0: Lung Segmentation in COVID-19 Computed Tomography Scans Using Hybrid Deep Learning Artificial Intelligence Models. Diagnostics 2021, 11, 1405. [CrossRef] [PubMed]

55. He, K.; Zhang, X.; Ren, S.; Sun, J. Deep residual learning for image recognition. In Proceedings of the IEEE Conference on Computer Vision and Pattern Recognition, Las Vegas, NV, USA, 27-30 June 2016; IEEE: Piscataway, NJ, USA, 2016; pp. 770-778.

56. Acharya, U.R.; Faust, O.; Sree, S.V.; Molinari, F.; Saba, L.; Nicolaides, A.; Suri, J.S. An accurate and generalized approach to plaque characterization in 346 carotid ultrasound scans. IEEE Trans. Instrum. Meas. 2012, 61, 1045-1053. [CrossRef]

57. Acharya, U.R.; Saba, L.; Molinari, F.; Guerriero, S.; Suri, J.S. Ovarian tumor characterization and classification: A class of GyneScan $^{\mathrm{TM}}$ systems. In Proceedings of the 2012 Annual International Conference of the IEEE Engineering in Medicine and Biology Society, San Diego, CA, USA, 28 August-1 September 2012; IEEE: Piscataway, NJ, USA, 2012.

58. Araki, T.; Ikeda, N.; Dey, N.; Acharjee, S.; Molinari, F.; Saba, L.; Godia, E.C.; Nicolaides, A.; Suri, J.S. Shape-based approach for coronary calcium lesion volume measurement on intravascular ultrasound imaging and its association with carotid intima-media thickness. J. Ultrasound Med. 2015, 34, 469-482. [CrossRef]

59. Barqawi, A.B.; Li, L.; Crawford, E.D.; Fenster, A.; Werahera, P.N.; Kumar, D.; Miller, S.; Suri, J.S. Three different strategies for real-time prostate capsule volume computation from 3-D end-fire transrectal ultrasound. In Proceedings of the 2007 29th Annual International Conference of the IEEE Engineering in Medicine and Biology Society, Lyon, France, 22-26 August 2007; IEEE: Piscataway, NJ, USA, 2007.

60. Suri, J.S.; Haralick, R.M.; Sheehan, F.H. Left ventricle longitudinal axis fitting and its apex estimation using a robust algorithm and its performance: A parametric apex model. In Proceedings of the International Conference on Image Processing, Santa Barbara, CA, USA, 14-17 July 1997; IEEE: Piscataway, NJ, USA, 1997.

61. Singh, B.K.; Verma, K.; Thoke, A.S.; Suri, J.S. Risk stratification of 2D ultrasound-based breast lesions using hybrid feature selection in machine learning paradigm. Measurement 2017, 105, 146-157. [CrossRef]

62. Riffenburgh, R.H.; Gillen, D.L. Contents. In Statistics in Medicine; Academic Press: Cambridge, MA, USA, 2020; pp. ix-xvi.

63. Acharya, R.U.; Faust, O.; Alvin, A.P.C.; Sree, S.V.; Molinari, F.; Saba, L.; Nicolaides, A.; Suri, J.S. Symptomatic vs. asymptomatic plaque classification in carotid ultrasound. J. Med. Syst. 2012, 36, 1861-1871. [CrossRef]

64. Acharya, U.R.; Vinitha Sree, S.; Mookiah, M.R.K.; Yantri, R.; Molinari, F.; Zieleźnik, W.; Małyszek-Tumidajewicz, J.; Stępień, B.; Bardales, R.H.; Witkowska, A.; et al. Diagnosis of Hashimoto's thyroiditis in ultrasound using tissue characterization and pixel classification. Proc. Inst. Mech. Eng. Part H J. Eng. Med. 2013, 227, 788-798. [CrossRef]

65. Acharya, U.R.; Faust, O.; Alvin, A.P.C.; Krishnamurthi, G.; Seabra, J.C.; Sanches, J.; Suri, J.S. Understanding symptomatology of atherosclerotic plaque by image-based tissue characterization. Comput. Methods Programs Biomed. 2013, 110, 66-75. [CrossRef]

66. Acharya, U.R.; Faust, O.; Sree, S.V.; Alvin, A.P.C.; Krishnamurthi, G.; Sanches, J.; Suri, J.S. Atheromatic ${ }^{\mathrm{TM}}$ : Symptomatic vs. asymptomatic classification of carotid ultrasound plaque using a combination of HOS, DWT \& texture. In Proceedings of the 2011 Annual International Conference of the IEEE Engineering in Medicine and Biology Society, Boston, MA, USA, 3 August-3 September 2011; IEEE: Piscataway, NJ, USA, 2011. 
67. Acharya, U.R.; Mookiah, M.R.K.; Sree, S.V.; Afonso, D.; Sanches, J.; Shafique, S.; Nicolaides, A.; Pedro, L.M.; e Fernandes, J.F.; Suri, J.S. Atherosclerotic plaque tissue characterization in 2D ultrasound longitudinal carotid scans for automated classification: A paradigm for stroke risk assessment. Med. Biol. Eng. Comput. 2013, 51, 513-523. [CrossRef]

68. Molinari, F.; Liboni, W.; Pavanelli, E.; Giustetto, P.; Badalamenti, S.; Suri, J.S. Accurate and automatic carotid plaque characterization in contrast enhanced 2-D ultrasound images. In Proceedings of the 29th Annual International Conference of the IEEE Engineering in Medicine and Biology Society, Lyon, France, 22-26 August 2007; IEEE: Piscataway, NJ, USA, 2007.

69. Saba, L.; Biswas, M.; Suri, H.S.; Viskovic, K.; Laird, J.R.; Cuadrado-Godia, E.; Nicolaides, A.; Khanna, N.N.; Viswanathan, V.; Suri, J.S. Ultrasound-based carotid stenosis measurement and risk stratification in diabetic cohort: A deep learning paradigm. Cardiovasc. Diagn. Ther. 2019, 9, 439-461. [CrossRef]

70. Biswas, M.; Kuppili, V.; Saba, L.; Edla, D.R.; Suri, H.S.; Sharma, A.; Cuadrado-Godia, E.; Laird, J.R.; Nicolaides, A.; Suri, J.S. Deep learning fully convolution network for lumen characterization in diabetic patients using carotid ultrasound: A tool for stroke risk. Med Biol. Eng. Comput. 2019, 57, 543-564. [CrossRef]

71. Chaddad, A.; Hassan, L.; Desrosiers, C. Deep CNN models for predicting COVID-19 in CT and x-ray images. J. Med. Imaging 2021, 8 (Suppl. S1), 014502. [CrossRef] [PubMed]

72. Gunraj, H.; Wang, L.; Wong, A. COVIDNet-CT: A Tailored Deep Convolutional Neural Network Design for Detection of COVID-19 Cases From Chest CT Images. Front. Med. 2020, 7, 608525. [CrossRef] [PubMed]

73. Iyer, T.J.; Raj, A.N.J.; Ghildiyal, S.; Nersisson, R. Performance analysis of lightweight CNN models to segment infectious lung tissues of COVID-19 cases from tomographic images. PeerJ Comput. Sci. 2021, 7, e368. [CrossRef]

74. Ranjbarzadeh, R.; Jafarzadeh Ghoushchi, S.; Bendechache, M.; Amirabadi, A.; Ab Rahman, M.N.; Baseri Saadi, S.; Aghamohammadi, A.; Kooshki Forooshani, M. Lung Infection Segmentation for COVID-19 Pneumonia Based on a Cascade Convolutional Network from CT Images. BioMed Res. Int. 2021, 2021, 5544742. [CrossRef]

75. Erasmus, J.J.; Gladish, G.W.; Broemeling, L.; Sabloff, B.S.; Truong, M.T.; Herbst, R.S.; Munden, R.F. Interobserver and intraobserver variability in measurement of non-small-cell carcinoma lung lesions: Implications for assessment of tumor response. J. Clin. Oncol. 2003, 21, 2574-2582. [CrossRef]

76. Joskowicz, L.; Cohen, D.; Caplan, N.; Sosna, J. Inter-observer variability of manual contour delineation of structures in CT. Eur. Radiol. 2019, 29, 1391-1399. [CrossRef] [PubMed]

77. El-Baz, A.; Suri, J. Lung Imaging and CADx; CRC Press: Boca Raton, FL, USA, 2019.

78. El-Baz, A.; Suri, J.S. Lung Imaging and Computer Aided Diagnosis; CRC Press: Boca Raton, FL, USA, 2011.

79. Sudeep, P.V.; Palanisamy, P.; Rajan, J.; Baradaran, H.; Saba, L.; Gupta, A.; Suri, J.S. Speckle reduction in medical ultrasound images using an unbiased non-local means method. Biomed. Signal Process. Control. 2016, 28, 1-8. [CrossRef]

80. Sarker, M.M.K.; Rashwan, H.A.; Akram, F.; Singh, V.K.; Banu, S.F.; Chowdhury, F.U.; Choudhury, K.A.; Chambon, S.; Radeva, P.; Puig, D.; et al. SLSNet: Skin lesion segmentation using a lightweight generative adversarial network. Expert Syst. Appl. 2021, 183, 115433. [CrossRef]

81. Saba, L.; Agarwal, M.; Sanagala, S.S.; Gupta, S.K.; Sinha, G.R.; Johri, A.M.; Khanna, N.N.; Mavrogeni, S.; Laird, J.R.; Pareek, G.; et al. Brain MRI-based Wilson disease tissue classification: An optimised deep transfer learning approach. Electron. Lett. 2020, 56, 1395-1398. [CrossRef]

82. El-Baz, A.; Suri, J.S. Big Data in Multimodal Medical Imaging; CRC Press: Boca Raton, FL, USA, 2019. 